\title{
QUANTUM ENVIRONMENTS: SPINS BATHS, OSCILLATOR BATHS, and applications to QUANTUM MAGNETISM
}

\author{
P.C.E. STAMP \\ Physics Department, and Canadian Institute for Advanced Research, \\ University of British Columbia, 6224 Agricultural Rd., Vancouver, B.C., Canada V6T 1Z1 \\ and \\ L.C.M.I./ Max Planck Institute, Ave. des Martyrs, Grenoble 38042, France \\ $* * *$ \\ email: stamp@physics.ubc.ca
}

September 3, 2018

\begin{abstract}
The low-energy physics of systems coupled to their surroundings is understood by truncating to effective Hamiltonians; these tend to reduce to a few canonical forms, involving coupling to "baths' of oscillators or spins. The method for doing this is demonstrated using examples from magnetism, superconductivity, and measurement theory, as is the way one then solves for the low-energy dynamics. Finally, detailed application is given to the exciting recent Quantum Relaxation and tunneling work in nanomagnets.
\end{abstract}

\section{Introduction}

These lecture notes are taken from lectures given in April 1997 at the Institute for Nuclear Theory in Seattle (University of Washington). Their main point was to explain how condensed matter theorists deal with problems in which some degree/degrees of freedom of interest, are coupled to a background "quantum environment". In almost all cases the interesting degrees of freedom are showing quantum behaviour, and often they are mesocopic or even macroscopic.

The tactic adopted in the lectures was to explain the general framework within which the theory operates, and then to demonstrate its operation with the help of examples. These examples are taken from various fields (including superconductivity, the Kondo problem, and one-dimensional systems), but the emphasisis is on the very active new field of "Quantum Nanomagnetism". Great scientific interest has been generated (as well as some rather misleading articles in the more popular press) by recent experiments in crystals of magnetic macromolecules [1, 2, 3, 4, 5, 6, 6], which show resonant tunneling of their magnetisation. Other experiments which have also generated considerable press include coherence experiments on the ferritin biological macromolecule [8, 9], and tunneling experiments on domain walls in magnetic wires [10, 11]. I thus spent some time explaining how the theoretical methods may be applied to these real systems. For another very important example of large-scale quantum behaviour, see the lectures of A.J. Leggett in this volume, who discusses macroscopic quantum phenomena in superconducting systems. 
The notes are divided into 3 parts. The first part (Chapter 2) deals with the essential theoretical step in which one sets up a low-energy description of the system of interest. The result of this is an "effective Hamiltonian", which claims to accurately describe both the quantum degrees of freedom of interest, and the quantum environment (and their coupling), below a certain energy cut-off. We end up with 2 kinds of quantum environment. One is the oscillator bath model, which is very old - in its modern form it goes back to papers of Feynman et al. [12], at the beginning of the 1960's. Except in rather unusual cases, it well describes an environment of delocalized modes (eg. phonons, photons, conduction electrons, magnons, etc). It was introduced in this form into the discussion of tunneling by Caldeira and Leggett 13] in 1981. The second environment is the "spin bath" model, which has been developed recently by myself and Prokof'ev [14, 15, 16, 17, 18] (see also refs. [19, 21, 20, 221), stimulated particularly by problems in quantum nanomagnetism (although it can be applied outside this domain [21, 22, 23, 24]; to illustrate this, brief space is given to superconducting SQUIDs and spin chains). It describes environmental modes like paramagnetic or nuclear spins, or other similar cases in which each environmental mode has only a few (often only two) levels of interest, and where they are very weakly coupled. The main point of this chapter is that readers may see clearly how the "truncation" to an effective Hamiltonian works, and to carry it out explicitly on some model examples in a tutorial way.

Once one has an effective Hamiltonian, the next task (chapter 3) is to solve for the dynamics of the degrees of freedom we are interested in, by "integrating out" (averaging over) the environmental modes. Doing this for an oscillator bath is standard, so I don't spend too much time on it, except to delineate the main ideas and results. More time is spent on the spin bath, where some new tricks are needed, and where the results are often radically different from the oscillator bath. Technical details are kept to a minimum, and ideas are emphasized. Again, it is then shown how these results apply to some real systems.

In both Chapters 2 and 3, I try to show how a very wide variety of physical problems tends to reduce to just a very few "canonical models" at low energies. Many readers will not be surprised by this- it is of course the idea underlying the whole philosophy of "universality classes" and the renormalisation group.

Finally in the 3rd part of these lectures (chapter 4) I turn to some real down-to-earth physics, and discuss recent advances in our understanding of quantum magnets, particularly at the nano/mesoscopic level, where all sorts of interesting tunneling phenomena occur. These include simple dissipative tunneling and quantum relaxation; one also gets resonant tunneling relaxation and there is evidence for quantum coherence at the mesoscopic level. Although not all experiments are properly understood at present, it is becoming very clear that much of the physics is controlled by the combined effect of nuclear spins and magnetic dipolar fields on the tunneling entities; moreover, it is essential to take proper account of the nuclear dynamics, which justifies a posteriori the interest in the spin bath model. There is a rich experimental literature here, on magnetic molecules and particles, and on magnetic wires, as well as related experiments on spin chains and on the "colossal resistance" Mn- perovskite materials.

¿From this summary the reader will see that I neglect discussion of other currently-used models of quantum environments such as the "Landau-Zener" model 19, 25, 26. This is unfortunate, because it is much easier to connect this model with studies in quantum chaos, then the "bath" models I have described. My only excuses are (i) lack of space, and (ii) lack of experience - condensed matter physicists are typically forced to deal with real solids, liquids, or glasses, in which statistical averages over environmental modes are unavoidable. 


\section{EFFECTIVE HAMILTONIANS: SPIN BATHS AND OSCILLATOR BATHS}

In this first lecture I discuss, from both the physical and mathematical points of view, a rather extraordinary simplifying feature of almost all physical problems, which is fundamental to our understanding of complex systems having many degrees of freedom. It is simply this - that provided we are only interested in a certain energy scale (or a limited range of energies), we may throw away most of the degrees of freedom - they do not play any direct role in the physics in the energy range of interest. Of course this can't be done willy-nilly; it must be done carefully, and those degrees of freedom which have been dumped will still play an indirect role in the physics.

Readers will of course be familiar with this idea in many forms - one's first encounter with it, as a student, is often in the form of the "coarse-graining" assumptions inherent in statistical mechanics or thermodynamics. In this lecture we begin by showing how one actually carries out the truncation of the Hamiltonian of a complex system down to a low-energy "effective Hamiltonian". This is done explicitly for one real physical example, in some detail, so that readers can see what is involved.

Another important idea, also rather extraordinary, is that if one carries out such truncations for many different physical systems, the same kinds of low-energy effective Hamiltonian recur again and again. These "universality classes" thus become very important, and the corresponding effective Hamiltonians become worthy of study in their own right. In this lecture we study 2 such Hamiltonians. One is the "oscillator bath" model, in which some simple central system is coupled to an environment of harmonic oscillators. The simplest such central system is a single 2-level system; the model is then the famous "spin-boson" model. We will also look at a model in which two central spin systems couple to the oscillator bath (the "PISCES" model, where "PISCES" is an acronym for "Pair of Interacting Spins Coupled to an Environmental Sea"); and I mention a number of other such "canonical" models.

The other model effective Hamiltonian we shall study is the "spin-bath" model, where now the environment itself is represented by a set of 2-level systems. The simplest model of this kind is the "central spin" model, where the central system is also represented by a 2-level system. In the same way as for the oscillator bath environments, one can also develop a number of canonical spin bath models. Typically the spin bath models arise for environments of localised modes, whereas the oscillator baths represent delocalised modes.

I will discuss various examples in this lecture, to illustrate how these simple archetypal models really do represent the low-energy physics of a huge variety of real systems. What I will not do here is discuss the dynamics of these models- this is saved for the next lecture (section 3).

\subsection{Truncation to Low Energies}

The idea of a low-energy "truncated" effective Hamiltonian (or Lagrangian) goes back a long way - in classical mechanics to hydrodynamics, and in quantum theory to the old spin Hamiltonians. In its modern form (partially inspired by Landau's treatment of turbulence ) it is often discussed in the renormalization group (RNG) framework [27]. For the purposes of these lectures, we can formulate the problem as follows.

Typically one is presented with a reasonably accurately known "high-energy" or "bare" Hamiltonian (or Lagrangian) for a quantum system, valid below some "ultraviolet" upper energy cut-off energy $E_{c}$, and having the form

$$
\tilde{H}_{\text {Bare }}=\tilde{H}_{o}(\tilde{P}, \tilde{Q})+\tilde{H}_{\text {int }}(\tilde{P}, \tilde{Q} ; \tilde{p}, \tilde{q})+\tilde{H}_{\text {env }}(\tilde{p}, \tilde{q}) \quad\left(E<E_{c}\right),
$$

where $\tilde{Q}$ is an $\tilde{M}$-dimensional coordinate describing that part of the system we are interested in (with $\tilde{P}$ the corresponding conjugate momentum), and $(\tilde{p}, \tilde{q})$ are $\tilde{N}$-dimensional coordinates describing all other degrees of freedom 
which may couple to $(\tilde{P}, \tilde{Q})$. Conventionally one refers to $(\tilde{p}, \tilde{q})$ as environmental coordinates.

What is important about $\tilde{H}_{\text {Bare }}$ is that its form is known well (it can of course be regarded as a low-energy form of some other even higher-energy Hamiltonian, in a chain extending ultimately back to quarks and leptons). If, however, one is only interested in physics below a much lower energy scale $\Omega_{o}$, then the question is - how can we find a new effective Hamiltonian, having the form

$$
H_{\mathrm{eff}}=H_{o}(P, Q)+H_{\mathrm{int}}(P, Q ; p, q)+H_{\mathrm{env}}(p, q) \quad\left(E<\Omega_{o}\right)
$$

in the truncated Hilbert space of energies below $\Omega_{o}$ ? In this $H_{\text {eff }}, P$ and $Q$ are generalised $M$-dimensional coordinates of interest, and $p, q$ are $N$-dimensional environmental coordinates coupled to them. Since we have truncated the total Hilbert space, we have in general that $M<\tilde{M}$ and $N<\tilde{N}$. This truncation is illustrated in Fig. 1.

FIG. 1: The truncation Procedure (schematic). In (a) we see how the Hilbert space of the high- energy Hamiltonian (with UV cut-off $E_{c}$ ) is truncated down to low energies (UV cut-off $\Omega_{o}$ ), both for the system and the environment. In (b) the corresponding flow in the coupling constant space of effective Hamiltonians (here shown for 2 couplings $\alpha_{1}$ and $\alpha_{2}$ ).

At first glance it is not at all obvious why anyone would want to make this truncation, because its inevitable effect is to generate various couplings between the low-energy modes which were not there before. However there are 2 very good reasons for truncating. First, in spite of the new couplings appearing at low energy, it almost always turns out that $H_{\text {eff }}$ is easier to handle than $\tilde{H}_{\text {Bare }}$ (particularly in predicting the dynamics of the low-energy variables $P, Q$ of interest), simply because there are fewer variables to deal with. Second, and much more fundamental, the truncation pushes the new $H_{\text {eff }}$ towards some low-energy "fixed point" Hamiltonian (Fig. 1 again). Moreover, many different physical systems may "flow" to the same fixed point. This is particularly true for that part of $H_{\text {eff }}$ which describes the environment, and this allows theorists to speak of "universality classes" of quantum environment, each describing many different physical systems. Each of these different physical systems will then flow towards the same fixed point, and they will all be described by the same form for $H_{\text {env }}(p, q)$, albeit with different values for the 
couplings. In fact the low-energy couplings parametrize the position of a given system, with a given UV cut-off, in the space of available couplings ("effective Hamiltonian" space). As one varies the UV cut-off, the couplings change and any given system moves in the coupling space; but all systems in a given universality class move towards one fixed point as the UV cutoff $\Omega_{0}$ is reduced.

In this context one understands the various coupling terms in $H_{e f f}$, as a parametrisation of the path taken by $H_{e f f}$, as it approaches the fixed point.

¿From this point of view it is not so surprising that the description of quantum environments reduces to the discussion of just a few "universality classes". I emphasize this just because physicists are often quite surprised that one can discuss such a wide variety of, eg., tunneling problems with such a restricted class of models. Of course, in asserting that these fixed points exist, I have simply swapped one mystery for another - instead of scratching our heads over the way in which so many physical systems resemble each other in low energies, we are now left wondering why there must be a flow of $H_{\text {eff }}$ towards one or other fixed point (ie., why fixed points?). This is often how physics proceeds, by substituting one conundrum for another (albeit a more precisely formulated one!).

Let us leave this question hanging for now, and simply note here the enormous pragmatic interest of this result. We shall see, in the 2 universality classes of interest, that we can parametrise very simply the form of the low-energy physics. In the case of oscillator bath models, this will be in terms of a function $J(\omega)$, where $\omega$ is a frequency - this is the famous Caldeira-Leggett spectral function 13, which convolves the coupling to the bath with the bath density of states. In the case of spin bath models, one has a density of states function $W(\epsilon)$ for the spin bath (usually Gaussian), and parameters which describe both the coupling to the spin bath, and spin diffusion within the spin bath. At low temperatures and low applied fields, we will only care about the low-energy behaviour of $\omega$ or $\epsilon$. This will allow us in some cases to make rather sweeping statements about whole classes of physical systems (as well as calculating their detailed dynamics, for which see the next lecture, in Ch. 3). To take an example, we will be able to make remarks about quantum measurements which apply to large classes of measuring devices and measured systems, and yet which are still realistic (in the sense that they apply to real physical systems, instead of just to some idle toy model).

Having said this, I emphasize that a lot of the physics is in the details (a fact often ignored in the quantum measurement literature!). Thus, rather than giving some (necessarily rather abstract) attempt at a general discussion of the derivation and validity of these 2 models, I will instead show how it is done for some particular real systems. After this, some at least of the general features will be evident (as well as the pitfalls in a too general approach!).

\subsection{Spin Baths and Nanomagnets- a worked example}

Let's begin our formal approach to the truncation procedure, by picking a particular example of a physical system which reduces to a spin bath model at low energies. We shall look at a "nanomagnet", by which we mean a magnetically-ordered particle or molecule which is sufficiently small that all the electronic spins causing the magnetism are aligned along one axis (note that a macroscopic magnet does not satisfy this criterion - it is often full of domains, with spins pointing in various directions; and spin waves or other spin fluctuations can also cause the spin axis to wander around a macroscopic sample). The size of such monodomain particles does not often exceed $2000 \AA$; yet a particle this large can contain as many as $10^{8}$ electronic spins! Because all microscopic spins are aligned, the total system then behaves like a "giant spin", denoted by $\vec{S}$.

As we shall see, the most important environment to which $\vec{S}$ is coupled is the set of nuclear spins inside it (and often to nuclear and paramagnetic impurity spins outside it as well). This is how the spin bath arises. Our aim in this section is to truncate this physical system to low energies. Recall that the reason for going through this is so 
you can see how it works on a realistic example. You don't have to care about nanomagnets to follow the exercise the point is to understand the truncation procedure, and see how to apply it elsewhere.

\section{2(a) HIGH ENERGY HAMILTONIAN}

Suppose we start by looking for a high-energy effective Hamiltonian, analogous to (2.1), but for a magnetic system which is coupled to a set of weakly-coupled spin-1/2 spins (ie., a "spin bath", instead of an "oscillator bath"). If the magnetic degrees of freedom are described by some continuous variable $Q$, and the oscillator coordinates $(\tilde{p}, \tilde{q})$ are replaced by a set of $N$ spin-1/2 variables $\left\{\hat{\vec{\sigma}}_{k}\right\}$, i.e., two-level systems, we get a high-energy Hamiltonian of general form

$$
\begin{gathered}
H=H_{o}(P, Q)+H_{\mathrm{int}}(P, Q ;\{\hat{\vec{\sigma}}\})+H_{\mathrm{env}}(\{\hat{\vec{\sigma}}\}) ; \\
H_{\mathrm{int}}(P, Q ;\{\hat{\vec{\sigma}}\})=\sum_{k=1}^{N}\left[F_{\|}(P, Q) \hat{\sigma}_{k}^{z}+\left(F_{\perp}(P, Q) \hat{\sigma}_{k}^{-}+h . c .\right)\right] ; \\
H_{\mathrm{env}}(\{\hat{\vec{\sigma}}\})=\sum_{k=1}^{N} \vec{h}_{k} \cdot \hat{\vec{\sigma}}_{k}+\frac{1}{2} \sum_{k=1}^{N} \sum_{k^{\prime}=1}^{N} V_{k k^{\prime}}^{\alpha \beta} \hat{\sigma}_{k}^{\alpha} \hat{\sigma}_{k^{\prime}}^{\beta},
\end{gathered}
$$

for energy scales $E<E_{c}$. In this Hamiltonian, we have a "central system", moving in a space described by the continuous coordinate $Q$, which couples simultaneously to the environmental spin variables $\left\{\hat{\vec{\sigma}}_{k}\right\}$. An example of such a Hamiltonian, which has been worked out in detail recently, is that for a magnetic soliton, like a domain wall, at position $Q$, coupled by hyperfine interactions to a set of $N$ spin-1/2 nuclear spins [28]; this example will be discussed in section 2.3(b). In the case of nuclear spins $V_{k k^{\prime}}^{\alpha \beta}$ describes the extremely weak internuclear dipolar coupling; typically $\left|V_{k k^{\prime}}^{\alpha \beta}\right| \leq 10^{-7} K$; and $\vec{h}_{k}$ is any external field that might influence these nuclei.

However in the case of nanomagnetic grains or magnetic macromolecules things simplify a great deal, because the continuous coordinate $Q$ can be replaced by a "giant spin" vector moving in the compact space of the surface of a sphere; we get a high-energy Hamiltonian of form

$$
H(\vec{S} ;\{\hat{\vec{\sigma}}\})=H_{o}(\vec{S})+\frac{1}{S} \sum_{k=1}^{N} \omega_{k} \vec{S} \cdot \hat{\vec{\sigma}}_{k}+H_{\mathrm{env}}(\{\hat{\vec{\sigma}}\}) ;
$$

where $H_{o}(\vec{S})$ is a "giant spin" Hamiltonian, describing a quantum rotator with spin quantum number $S=|\vec{S}| \gg 1$, and $H_{\text {env }}(\{\hat{\vec{\sigma}}\})$ is the same as in (5).

The assumption here is that we have a "monodomain" magnetic particle or macromolecule, in which the very strong exchange interactions lock the microscopic electronic spins $\vec{s}_{j}$ into either a giant ferromagnetic (FM) moment, with $\vec{S}=\sum_{j} \vec{s}_{j}$ (summed over ionic spin sites) or a giant antiferromagnetic (AFM) Néel vector, with $\vec{N} \equiv \vec{S}=\sum_{j}(-1)^{j} \vec{s}_{j}$ (for a simple staggered Néel order). With this assumption, the usual hyperfine coupling to the nuclear spins $\vec{I}_{k}$ can be written

$$
H_{H y p}=\sum_{k=1}^{N} \omega_{k} \underline{\mathbf{s}}_{k} \cdot \underline{\mathrm{I}}_{k} \rightarrow \frac{1}{S} \sum_{k=1}^{N} \omega_{k} \underline{\mathrm{S}} \cdot \underline{\mathrm{I}}_{k}
$$

(or a more complicated tensor interaction if we wish). In these lectures I will assume that $\left|\vec{I}_{k}\right|=I=\frac{1}{2}$, and write $\vec{I}_{k} \rightarrow \sigma_{k}$, i.e. the nuclear spins will be described by spin- $\frac{1}{2}$ Pauli matrices. In many cases even if $I \neq \frac{1}{2}$, the 
low-energy nuclear spin dynamics is well described by a 2-level system; the effect of the other levels appears in the field $\vec{h}_{k}$ in (5).

The "giant spin" Hamiltonian $H_{o}(\vec{S})$ itself can either be written as a continuous function of the vector $\vec{S}$ (assuming that $S$ is constant), or in terms of the usual spin operators for $\vec{S}$, for which we have the general form

$$
H_{o}(\underline{\mathrm{S}})=\sum_{l=1}^{S} \frac{{ }^{\|} K_{l}}{S^{l-1}} \hat{S}_{z}^{l}+\frac{1}{2} \sum_{r=1}^{S} \frac{{ }^{\perp} K_{r}}{S^{r-1}}\left(\hat{S}_{+}^{r}+\hat{S}_{-}^{r}\right)
$$

This form of the Hamiltonian separates the longitudinal part $\| \mathcal{H}\left(\hat{S}_{z}\right)$ (which conserves $S_{z}$ ) from the transverse part ${ }^{\perp} \mathcal{H}\left(\hat{S}_{+}, \hat{S}_{-}\right)$. In the symmetric case (all $l$ even) a pioneering semi-classical study of (1.8) was carried out by van Hemmen et al. 29, 30. A very simple example of the giant spin Hamiltonian in the symmetric case is the biaxial form:

$$
H_{o}(S)=\frac{1}{S}\left[{ }^{\|} K_{2} \hat{S}_{z}^{2}+{ }^{\perp} K_{2} \hat{S}_{y}^{2}\right]
$$

in which tunneling between the classical minima (at $S_{z}= \pm S$ ) is accomplished by the symmetry-breaking transverse ${ }^{\perp} K_{2}$ term, if ${ }^{\|} K_{2}$ is negative.

FIG. 2: The excited states of a nanomagnet (schematic): The $2 S+1$ states of the giant spin appear at low energies. All states that break the constraint $\sum_{j} \vec{s}_{j}=\vec{S}$, with $|\vec{S}|=s$, are referred to as internal magnon states. As $S \rightarrow 0$, the energy at the bottom of the magnon band tends to the interspin exchange $J$, but it falls as $S$ increases, because the magnons can spread out. When it reaches the top of the giant spin manifold, the two manifolds mix strongly in the overlap region shown.

I refer here to the giant spin Hamiltonian for a nanomagnet as a "high-energy" Hamiltonian, but of course it is obvious that Hamiltonians like (8) or (9) are themselves the result of the truncation of an even higher energy 
(more "microscopic") electronic spin Hamiltonian like, eg.,

$$
H_{o}\left(\left\{\underline{\mathbf{s}}_{j}\right\}\right)=\frac{1}{2} \sum_{<i j>} J_{i j}^{\alpha \beta} \hat{s}_{i}^{\alpha} \hat{s}_{j}^{\beta}+\frac{1}{2} \sum_{j} K_{j}^{\alpha \beta} \hat{s}_{j}^{\alpha} \hat{s}_{j}^{\beta}
$$

If $\left|s_{j}\right|=s$ (so there are $L=S / s$ electronic spins), this Hamiltonian acts in a huge Hilbert space of dimension $(2 s+1)^{L}$. I would like to strongly emphasize here a practical point of some importance (which will be familiar to anyone working in mesoscopic or nanoscopic physics). Even if we knew all the couplings $J_{i j}^{\alpha \beta}, K_{j}^{\alpha \beta}$ (which is hardly likely given the internal complexity of most nanomagnets), truncation of $H_{o}(\underline{S})$ at low energies is practically impossible if $L>5-10$, even with supercomputers. Instead one attempts to measure the parameters in (8) or (9), thereafter treating them as "fundamental" (similar situations are encountered in most problems involving strong interactions, ranging from QCD to Fermi liquid theory). Moreover, even this is not easy - experiments such as ESR (Electron Spin Resonance) can only parametrize terms with small $\ell$ or $r$ in (8). Unfortunately the tunneling matrix element $\Delta_{S}$, between levels $\left|S_{z}>= \pm\right| S>$ of the longitudinal part of (8) is just as much influenced by higher transverse couplings as by lower ones (a point to be discussed in more detail in the last section). Thus in any practical situation, we will probably not know all of the important terms in our giant spin Hamiltonian.

In any case it is clear that $H_{o}(\vec{S})$ is only strictly meaningful if the higher states in $H_{o}\left(\left\{\vec{S}_{j}\right\}\right)$ are not excited; these "internal magnon" states (of which there are a huge number) break the constraint $\sum_{j} \vec{s}_{j}=S$ (or $\left.\sum_{j}(-1)^{j} \vec{s}_{j}=\vec{N}\right)$. What this implies is shown schematically in Fig. 2; as the nanomagnet gets bigger, internal magnon states (where internal spin flips overcome the powerful $J_{i j}$ exchange) can lower their energy by spreading over the whole sample. Once these start impinging on the $(2 S+1)$ - dimensional giant spin manifold, we may be in trouble. Note that the giant spin manifold is spread over $\sim S K$ in energy (where $K$ is the dominant anisotropy term in $H_{o}(\vec{S})$ ); values of $K$ are typically in the range $0.1-10 \mathrm{~K}$.

\section{2(b) TRUNCATION to LOW ENERGIES (QUANTUM REGIME)}

Suppose however that one is interested in energies or temperatures where only the 2 lowest levels of $H_{o}(\vec{S})$ are involved. This arises in particular when one is interested in quantum phenomena, since the dynamics of the system go over into a temperature-independent quantum regime once the higher levels can no longer be thermally activated. We are then at liberty, in the absence of the nuclear spins, to truncate $H_{o}(\vec{S})$ to a simple 2-level Hamiltonian $H_{o}(\vec{\tau})$, which is typically written as

$$
H_{o}(\vec{\tau})=\Delta_{o} \hat{\tau}_{x}+\xi \hat{\tau}_{x}
$$

where $\vec{\tau}$ is a Pauli spin. It is very important, in the context of macroscopic quantum phenomena, that $H_{o}(\vec{\tau})$ is meaningful below a UV cut-off energy $\Omega_{0} \sim K$ which is independent of $S$ (at least until the monodomain assumption fails, and we return to (10)). This means that we enter the quantum regime at temperatures which are often as high as several degrees Kelvin, even for very large spins.

Our essential task now becomes clear - we need to continue our truncation of (6) down to low T. For the simple giant spin Hamiltonian (no nuclear spins), the passage from (8) to (11) has been discussed by many authors for $S \gg 1$. Korenblit and Shender [31] used perturbation expansions (valid if ${ }^{\perp} \mathcal{H} / \|_{\mathcal{H}} \ll 1$ ); van Hemmen and Sutő [29] used WKB methods (valid for arbitrary ${ }^{\perp} \mathcal{H} / \|^{\|} \mathcal{H}$, provided $S$ is large enough), and Enz and Schilling introduced instanton methods [32] (in many cases, formally equivalent to the WKB method, but not so general - one requires a semiclassical path or paths). Quite a lot can (and has been) said about this kind of truncation, but the problem is now solved, and you can read about it in the literature. What interests us here is the fact that such calculations 
are basically irrelevant to the real world, because of the strong coupling to the nuclear spins. This is a much more complicated problem, involving many more degrees of freedom- yet such problems are quite generic in this game, since there is always going to be some environment which relaxes the system.

In what follows, I will describe how the truncation can be done using instanton methods. This is certainly not the only way one could imagine it being done, and in fact it would be very interesting to see it done otherwise. Since this course is pedagogical, I will not try to to the reduction for a general Hamiltonian; instead it is done for the simple biaxial Hamiltonian given above.

(i) Free Giant Spins: To warm up, lets briefly recap how the instanton analysis works for the simple isolated biaxial Hamiltonian (1.9) (cf. ref [32, 33, 34, 35]). We start by choosing a basis in the truncated (2-level) space such that the eigenstates of $\hat{\tau}_{z}$ correspond to the 2 semiclassical minimum states of $H_{o}(\vec{S})$, defined by coherent state vectors $\left|\vec{n}_{1}\right\rangle$ and $\left|\vec{n}_{2}\right\rangle$, such that $\left.\vec{n}_{1}|\vec{S}| \vec{n}_{1}\right\rangle=S \vec{n}_{1}$ and $\left.\vec{n}_{2}|\vec{S}| \vec{n}_{2}\right\rangle=S \vec{n}_{2}$; the eigenstates of $H_{\text {eff }}^{o}(\vec{\tau})$ are then linear combinations of $\left|\vec{n}_{1}\right\rangle$ and $\left|\vec{n}_{2}\right\rangle$, which we can determine once we have found the four matrix elements $\left\langle\vec{n}_{\alpha}\left|H_{e f f}^{o}\right| \vec{n}_{\beta}\right\rangle$ with $\alpha, \beta=1,2$.

Formally one can do this as follows, for the free spin. Consider the path integral expression for the transition amplitude $\Gamma_{\alpha \beta}^{o}(t)$, during the time $t$; this is given by []:

$$
\Gamma_{\alpha \beta}^{o}(t)=\left\langle\vec{n}_{\alpha}\left|e^{-i H_{o}(\vec{S}) t}\right| \vec{n}_{\beta}\right\rangle=\int_{\vec{n}(\tau=0)=\vec{n}_{\beta}}^{\vec{n}(\tau=t)=\vec{n}_{\alpha}} \mathcal{D} \vec{n}(\tau) \exp \left\{-\int_{0}^{t} d \tau \mathcal{L}_{o}(\tau)\right\},
$$

where the free spin Euclidean Lagrangian is

$$
\mathcal{L}_{o}=-i S \dot{\theta} \varphi \sin \theta+H_{o}(\vec{n})
$$

Here $\theta$ and $\varphi$ are the usual polar and azimuthal angles for the unit vector $\vec{n}(\tau)$.

Now in the semiclassical approximation there are two fundamental time scales in the paths $\vec{n}(\tau)$ in $(12)$; these are $\Omega_{o}^{-1}$, the time required for the instanton traversal to be made between states, and $\Delta_{o}^{-1}$, the typical time elapsing between instantons. By definition, an effective Hamiltonian is supposed to reproduce the slow dynamics of the system in the truncated Hilbert space of the two lowest levels, i.e., for long time scales an evolution operator is approximated as

$$
\Gamma_{\alpha \beta}^{o}(t) \approx\left(e^{-i H_{e f f} t}\right)_{\alpha \beta}
$$

Since $\Delta_{o}$ is exponentially smaller than $\Omega_{o}$, and the nondiagonal elements are $\sim \Delta_{o}$, we can write

$$
\begin{aligned}
\Gamma_{\alpha \beta}^{o}(t) & =\left\langle\vec{n}_{\alpha}\left|e^{-i H_{e f f} t}\right| \vec{n}_{\beta}\right\rangle \\
& \approx \delta_{\alpha \beta}-i t\left\langle\vec{n}_{\alpha}\left|H_{e f f}^{o}\right| \vec{n}_{\beta}\right\rangle ; \quad\left(\Omega_{o}^{-1} \ll t \ll \Delta_{o}^{-1}\right) ;
\end{aligned}
$$

Then we immediately find the matrix elements of $H_{\text {eff }}^{o}(\vec{\tau})$ for $\alpha \neq \beta$ as

$$
\left(H_{e f f}^{o}(\vec{\tau})\right)_{\alpha \beta}=\frac{i}{t} \Gamma_{\alpha \beta}^{o}(t) ; \quad\left(\Omega_{o}^{-1} \ll t \ll \Delta_{o}^{-1}\right)
$$

As a concrete example, consider the easy-axis/easy-plane Hamiltonian (9), where

$$
H_{o}(\vec{n})=S K_{\|}\left[\sin ^{2} \theta+\frac{K_{\perp}}{K_{\|}} \sin ^{2} \theta \sin ^{2} \varphi\right]
$$


The two lowest states are $\vec{n}_{1}=\hat{\vec{z}}$ and $\vec{n}_{2}=-\hat{\vec{z}}$; henceforth we write these states as $|\Uparrow\rangle$ and $|\Downarrow\rangle$. In the usual case where $K_{\perp} / K_{\|} \gg 1$ (so that the tunneling amplitude is appreciable) one has only small oscillations of $\varphi$ about the semiclassical trajectories $\varphi=0$ or $\pi$, and by eliminating $\varphi$ one has

$$
\mathcal{L}_{o}(\theta)=\frac{S}{4 K_{\perp}} \dot{\theta}^{2}+S K_{\|} \sin ^{2} \theta
$$

giving a classical equation of motion $\dot{\theta}=\Omega_{o} \sin \theta$, and instanton solution [36, 37], going from $|\Uparrow\rangle$ to $|\Downarrow\rangle$

$$
\sin \theta(\tau)=1 / \cosh \left(\Omega_{0} \tau\right)
$$

(with the instanton centered at $\tau=0$ ); in this system the "bounce" or "small oscillation" frequency is

$$
\Omega_{o}=2\left(K_{\|} K_{\perp}\right)^{1 / 2} .
$$

The frequency $\Omega_{o}$ then sets the ultraviolet cut-off for the Hilbert space of $H_{\text {eff }}^{o}(\vec{\tau})$, and one finds, by substituting the semiclassical solution into (12) and evaluating the determinant over the quadratic fluctuations around the semiclassical solution 38 (the zero mode contribution gives a factor $i t$, in the usual way), that from (16) we get [29, 32, 33, 34, 36, 37, 39]:

$$
\begin{gathered}
\hat{H}_{\text {eff }}^{o}(\vec{\tau})=\frac{\Delta_{o}(S)}{2} \hat{\tau}_{x} \\
\Delta_{o}(S)=-\sum_{\eta= \pm} \sqrt{\frac{2}{\pi} \operatorname{Re} A_{o}^{(\eta)}} \Omega_{o} \exp \left\{-A_{o}^{(\eta)}\right\} \equiv 2 \Delta_{o} \cos \pi S \\
A_{o}^{(\eta)}=2 S\left(K_{\|} / K_{\perp}\right)^{1 / 2}+i \eta \pi S
\end{gathered}
$$

where the action $A_{o}^{(\eta)}$ is that for the transition between the two semiclassical minima, either clockwise $(\eta=+)$ or anticlockwise $(\eta=-)$; the phase $\eta \pi S$ is the Kramers/Haldane phase, coming from the linear in time derivatives kinetic term in (13). Without this phase, we would simply have a splitting $\left|\Delta_{o}\right|=\sqrt{2 R e A_{o} / \pi} \Omega_{o} \exp \left\{-A_{o}\right\}$ with $A_{o}=2 S\left(K_{\|} / K_{\perp}\right)^{1 / 2}$.

For this symmetric problem (where the 2 semiclassical states $|\Uparrow\rangle$ and $|\Downarrow\rangle$ are degenerate), the diagonal matrix elements in $\hat{H}_{e f f}^{o}$ are zero (in fact if we computed them directly, again by evaluating the derminant, we would find a value $\Omega_{o}$ for each- but this is just the energy of the 2 states measured from the bottom of each well, and it makes sense to redefine the energy zero to make these diagonal elements zero). If instead of considering the symmetric problem, I had also added an external bias field, causing an energy difference $\xi$ in the absence of tunneling, then the diagonal elements would simply be $\pm \xi / 2$, and we would end up with an effective Hamiltonian

$$
H_{\text {eff }}^{o}=\frac{\Delta_{0}}{2} \hat{\tau}_{x}+\frac{\xi}{2} \hat{\tau}_{z}
$$

with eigenvalues

$$
E_{ \pm}= \pm|E|= \pm \frac{1}{2} \sqrt{\Delta^{2}+\epsilon^{2}}
$$

and eigenfunctions

$$
\psi_{ \pm}=A_{ \pm}\left[\left(E_{ \pm}+\epsilon\right)|\Uparrow\rangle-\Delta_{0}|\Downarrow\rangle\right],
$$




$$
A_{ \pm}=\left[\frac{1}{\left(E_{ \pm}+\epsilon\right)^{2}+\left(\Delta_{0}\right)^{2}}\right]^{1 / 2}
$$

Suppose this 2-level system starts off at $t=0$ in the state $|\Uparrow\rangle$. Then after a time $t$, the system is described by a density matrix in the same basis, given by

$$
\rho(t)=\left(\begin{array}{cc}
1-\frac{\Delta_{0}^{2}}{E^{2}} \sin ^{2} E t & -i \frac{\Delta_{0}}{E} \sin E t \\
i \frac{\Delta_{0}}{E} \sin E t & \frac{\Delta_{0}^{2}}{E^{2}} \sin ^{2} E t
\end{array}\right)
$$

In the equivalent 2-well problem, the initial "wave-packet" $|\Uparrow\rangle$ partially oscillates between the 2 wells- the diagonal elements give the occupation probability of the wells, and the off-diagonal elements describe oscillatory quantum interference between them, which is suppressed by the bias; when $\xi \gg \Delta_{0}$, the system stays in one well, in the absence of any coupling to the environment, even if this means it is in an excited (high-energy) state. To remove this "blocking" of transitions to the lower energy state (and to give irreversible relaxation, as opposed to just oscillations), we need some kind of dynamic environment.

Notice in passing the limitations of this instanton derivation - it would not work in the absence of a few well-defined semiclassical paths. Thus if we replaced the transverse term ${ }^{\perp} K_{2} S_{y}^{2}$ by the rotationally invariant ${ }^{\perp} K_{2}\left(S_{x}^{2}+S_{y}^{2}\right) / 2$, there would be no favoured semiclassical path, and we would be forced back onto the WKB or perturbative analyses. However when we can use the instanton formalism, it is easily adaptable to include the spin bath as well; we now turn to this task.

(ii) Including the Spin Bath: I will not give all the details here (for which see Tupitsyn et al. [35]), just the main ideas. First, the basic physics. Before we couple the giant spin to the spin bath, the spin bath spectrum, containing $2^{N}$ lines, is almost completely degenerate - only the tiny internuclear coupling splits these lines. However the hyperfine coupling is very large (it ranges from $1.4 \mathrm{mK}$, in $\mathrm{Ni}$, to nearly $0.5 \mathrm{~K}$, in Ho, per nuclear spin, and it drastically alters the environmental spectrum. The nuclear levels, for a given giant spin state, now find themselves spread in a Gaussian multiplet of half-width $E_{o} \sim \omega_{o} N^{\frac{1}{2}}$ in energy (where $\omega_{o}$ is a typical hyperfine coupling) around the giant spin state; $E_{o}$ is many orders of magnitude greater than the width $T_{2}^{-1} \sim 10^{4}-10^{5} \mathrm{~Hz}$ of the nuclear multiplet before coupling. In this sense the nuclear spin bath degrees of freedom are slaved to the giant spin. Notice also that if the giant spin was formerly able to tunnel (because of near resonance between states $\mid S_{z}>$ and $\mid-S_{z}>$ ), this is unlikely now, because an extra internal bias field $\epsilon=\tau_{z} \Sigma_{k} \omega_{k} \sigma_{k}^{z}$ acts on $\underline{\tau}$ (indeed on $\vec{S}$ itself), and typically $\epsilon \gg \Delta_{o}$, pushing the giant spin way off resonance. Thus the hyperfine coupling to even a single nuclear spin drastically alters the giant spin dynamics as well. before doing anything formal, lets see qualitatively what must happen.

(i) In the absence of any nuclear dynamics this "degeneracy blocking" would only allow a tiny fraction of giant spins to make any tunneling transitions at all [14, 16, 17, 40] (Fig. 3). We will see in Chapter 4 that in the low temperature limit, it has been recently discovered that such giant spins can have very fast dynamics, which is (to me at any rate!) a very convincing demonstration of the role that the nuclear spin dynamics must play in the relaxation (since at these temperatures this is the only environment left with any dynamics at all!). This justifies $a$ posteriori the theoretical effort that has been put into understanding these effects!

(ii) A second effect of the spin bath is "topological decoherence" [14, 15, 16]. Each transition of the giant spin causes a time-dependent perturbation (via $H_{h y p}$ ) on the nuclear bath, which can cause transitions in the bath moreover, this perturbation causes a phase change in the nuclear bath state. Since this phase change varies from one transition of $\vec{S}$ to the next, the net effect is to randomize the phase of $\vec{S}$, ie., to cause phase decoherence. From the measurement point of view the nuclear spins are measuring time-varying fields due to $\vec{S}$ (ie., inhomogeneous in time 
rather than in space, as in the usual Stern-Gerlach experiment); this is a kind of "reverse Stern-Gerlach measuring apparatus" 22].

(iii) A final effect of the nuclear spins on the tunneling is that of "orthogonality blocking". Suppose that, semiclassically speaking, all nuclear spins are parallel/antiparallel to the net field acting on them (due to $\vec{S}$ and/or an external field), before $\vec{S}$ tunnels. What happens to them afterwards? The answer is that if they are still parallel/antiparallel to the new field, nothing at all. But in general the new field is not parallel/antiparallel to the old one, and so the bath spins must now precess (or quantum mechanically, make transitions) in the new field. The "mismatch" between old and new nuclear eigenstates causes a severe suppression of the tunneling of $\vec{S}$ (as well as decohering it).

Well, this all sounds very nice; but how do we deal formally with this physics? We wish to truncate to a low energy Hamiltonian $H_{\text {eff }}\left(\vec{\tau},\left\{\vec{\sigma}_{k}\right\}\right)$, valid for energies $\ll \Omega_{o}$ (cf. Fig. 1 ), where $\Omega_{o} \sim K$. First, let's cheat and look at the final answer! One finds for the general problem of a giant spin coupled to a spin bath that 14, 16, 35]

$$
\begin{aligned}
H_{\mathrm{eff}} & =\left\{2 \Delta_{o} \hat{\tau}_{-} \cos \left[\pi S-i \sum_{k}\left(\alpha_{k} \vec{n} \cdot \hat{\vec{\sigma}}_{k}+\beta_{o} \vec{n} \cdot \vec{H}_{o}\right)\right]+H . c .\right\} \\
& +\frac{\hat{\tau}_{z}}{2} \sum_{k=1}^{N} \omega_{k}^{\|} \vec{l}_{k} \cdot \hat{\vec{\sigma}}_{k}+\frac{1}{2} \sum_{k=1}^{N} \omega_{k}^{\perp} \vec{m}_{k} \cdot \hat{\vec{\sigma}}_{k}+\sum_{k=1}^{N} \sum_{k^{\prime}=1}^{N} V_{k k^{\prime}}^{\alpha \beta} \hat{\sigma}_{k}^{\alpha} \hat{\sigma}_{k^{\prime}}^{\beta} .
\end{aligned}
$$

I have also added an external field $\vec{H}_{o}$. This is the general form; we shall see below that for the biaxial nanospin, with the simple contact hyperfine interaction in (7), it simplifies somewhat. This effective Hamiltonian looks forbidding (although we shall see it is not so difficult to use); let us now look at each term in turn.

The first thing to notice is the separation into a diagonal term (in $\hat{\tau}_{z}$ ) and a non-diagonal one (in $\hat{\tau}_{+}, \hat{\tau}_{-}$). The non-diagonal term operates during transitions of $\vec{S}$, and it also causes transitions in the nuclear bath, because of the time-dependent field $\omega_{k} \vec{S} / S$ acting on each $\hat{\vec{\sigma}}_{k}$ during a transition. The diagonal term operates when $\vec{S}$ is in one of its two quiescent states $\vec{S}_{1}$ and $\vec{S}_{2}$. Defining as before the basis states $\left|\vec{n}_{1}>\equiv\right| \Uparrow>$ and $\left|\vec{n}_{2}>\equiv\right| \Downarrow>$, we have corresponding fields $\vec{\gamma}_{k}^{(1)}$ and $\vec{\gamma}_{k}^{(2)}$ acting on $\vec{\sigma}_{k}$. We define the sum and the difference terms as

$$
\begin{aligned}
\omega_{k}^{\|} \vec{l}_{k} & =\vec{\gamma}_{k}^{(1)}-\vec{\gamma}_{k}^{(2)} \\
\omega_{k}^{\perp} \vec{m}_{k} & =\vec{\gamma}_{k}^{(1)}+\vec{\gamma}_{k}^{(2)} .
\end{aligned}
$$

where the $\vec{l}_{k}$ and $\vec{m}_{k}$ are unit vectors. Then the truncated diagonal interaction takes the form (we project on states $|\Uparrow\rangle$ and $|\Downarrow\rangle$ using standard $\left(1+\hat{\tau}_{z}\right) / 2$ and $\left(1-\hat{\tau}_{z}\right) / 2$ operators $)$

$$
H_{\text {eff }}^{D}=\sum_{k=1}^{N}\left\{\vec{\gamma}_{k}^{(1)} \frac{1+\hat{\tau}_{z}}{2}+\vec{\gamma}_{k}^{(2)} \frac{1-\hat{\tau}_{z}}{2}\right\} \cdot \hat{\vec{\sigma}}_{k} \equiv \frac{1}{2}\left\{\hat{\tau}_{z} \sum_{k=1}^{N} \omega_{k}^{\|} \vec{l}_{k} \cdot \hat{\vec{\sigma}}_{k}+\sum_{k=1}^{N} \omega_{k}^{\perp} \vec{m}_{k} \cdot \hat{\vec{\sigma}}_{k}\right\},
$$

i.e., one term which changes when $\vec{S}_{1} \rightarrow \vec{S}_{2}$, and one which does not. Usually $\omega_{k}^{\|} \gg \omega_{k}^{\perp}$, and $\omega_{k}^{\|} \sim \omega_{o}$. Thus we get the diagonal term in (29). For the biaxial system with contact hyperfine interaction, the diagonal term is trivial; we get

$$
\omega_{k}^{\|}=\omega_{k} ; \quad \omega_{k}^{\perp}=0 ; \quad \text { (biaxial system) }
$$

Notice that it is this diagonal term which is responsible for the degeneracy blocking just mentioned- if we look at the combined density of states for the giant spin plus the nuclear levels, we see that there will be a huge multiplet 
of $2^{N}$ levels around each giant spin level. We will see later (section 3.4(a)) under what circumstances this takes the Gaussian form mentioned above.

Turning now to the non-diagonal term, we notice that unless $\omega_{k} \ll \Omega_{o}$, the hyperfine coupling itself can mediate interactions between the 2 lowest giant spin levels, and the higher levels. In practise $\omega_{k} / \Omega_{o} \ll 1$ almost always and we have a small parameter. Then instead of the bare transition matrix $\Gamma_{\alpha \beta}^{o}$ in (14), we calculate

$$
\begin{gathered}
\Gamma_{\alpha \beta}\left(\left\{\sigma_{k}^{(\alpha)}, \sigma_{k}^{(\beta)}\right\} ; t\right)= \\
\prod_{k=1}^{N} \int_{\vec{\sigma}_{k}^{(\alpha)}}^{\vec{\sigma}_{k}^{(\beta)}} \mathcal{D} \vec{\sigma}_{k}(\tau) \quad \int_{\vec{n}_{\alpha}}^{\vec{n}_{\beta}} \mathcal{D} \vec{n}(\tau) \exp \left\{-\int d \tau\left[\mathcal{L}_{o}(\tau)+\sum_{k=1}^{N} \mathcal{L}_{k}^{o}(\tau)+\delta \mathcal{L}_{\sigma}(\tau)\right]\right\} .
\end{gathered}
$$

where $\mathcal{L}_{k}^{o}(\tau)=-(i / 2) \dot{\Theta}_{k} \varphi_{k} \sin \Theta_{k}$ is the nuclear spin Lagrangian, and $\delta \mathcal{L}_{\sigma}(\tau)=\Sigma_{k=1} \vec{\gamma}_{k}(\tau) \cdot \vec{\sigma}_{k}(\tau)$, with $\vec{\gamma}_{k}(\tau)=$ $\omega_{k} \vec{S}(\tau) / 2 S$ the time-dependent field, from $\vec{S}$, acting on $\vec{\sigma}_{k}$. During the transition, $\vec{S}(\tau)$ varies over a timescale $\Omega_{o}^{-1}$, and so we get a time-dependent perturbation $\sim \omega_{k} / \Omega_{o}$ (ie., a sudden perturbation).

To calculate $\Gamma_{\alpha \beta}$ one first finds the new instanton trajectory by minimizing $\mathcal{L}_{o}(\tau)+\delta \mathcal{L}(\tau)$, and then calculates the transition amplitude from $\mid \vec{n}_{\alpha}>$ to $\mid \vec{n}_{\beta}>$; for details see Tupitsyn et al. [35. For the simple biaxial Hamiltonians (1.9), one gets for the transition element $\Gamma_{\Uparrow \Downarrow}(t)$ after a time $t$ (the "non-diagonal" amplitude):

$$
\hat{\Gamma}_{\Downarrow \Uparrow}(t)=i t \sum_{\eta= \pm} \sqrt{\frac{2}{\pi} \operatorname{Re} A_{o}} \Omega_{o} \exp \left\{-A_{o}^{(\eta)}-\eta \sum_{k}\left(\alpha_{k} \vec{n} \cdot \hat{\vec{\sigma}}_{k}+\beta_{o} \vec{n}_{o} \cdot \vec{H}_{o}\right)\right\} ; \quad\left(\Omega_{o}^{-1} \ll t \ll \Delta_{o}^{-1}\right)
$$

where the vectors $\alpha_{k} \vec{n}$ and $\beta_{o} \vec{n}_{o}$ have to be determined in terms of the parameters in the original high-energy Hamiltonian, in the course of minimizing the action. For the case of the biaxial giant spin Hamiltonian with contact hyperfine interactions, one easily gets

$$
\begin{aligned}
\alpha_{k} \vec{n} & =\frac{\pi \omega_{k}}{2 \Omega_{o}}\left(\hat{\vec{x}}, i \sqrt{K_{\|} / K_{\perp}} \hat{\vec{y}}\right) ; \\
\beta_{o} \vec{n}_{o} & =\frac{\pi \gamma_{e} S}{\Omega_{o}}\left(\hat{\vec{x}}, i \sqrt{K_{\|} / K_{\perp}} \hat{\vec{y}}\right),
\end{aligned}
$$

¿From this result the non-diagonal term in (29) follows immediately. The dimensionless parameter $\alpha_{k}$ is particularly interesting, since it parameterizes the inelastic effect, on the nuclear spin $\underline{\sigma}_{k}$, of transitions made by $\vec{S}$ (and it leads to topological decoherence [14). Note that it is complex, and that for $\omega_{k} / \Omega_{o} \ll 1$, we have $\left|\alpha_{k}\right| \sim \pi \omega_{k} / 2 \Omega_{o}$. Since the probability that $\vec{\sigma}_{k}$ will flip is $\frac{1}{2}\left|\alpha_{k}\right|^{2}$, this result is what we would expect (it follows from time-dependent perturbation theory in the sudden approximation). The only subtlety is that we cannot apply perturbation theory directly, since (at least in the instanton formalism), the tunneling takes place in imaginary time $\tau$ (not real time $t$ ); this is why the more detailed treatment of Tupitsyn et al. [35] is necessary. Roughly speaking, the real part of $\alpha$ adds an extra phase to the existing giant spin Berry phase (leading to topological decoherence), and the imaginary part is a renormalisation of the tunneling action caused by the nuclear spins.

This completes the discussion of how this particular spin bath effective Hamiltonian is derived - I have given details so you can see some of what is involved. In Chapter 3 we will see how to extract the dynamics of the giant spin (and we will then see the quantitative realisation of the concepts of topological decoherence, degeneracy blocking, and orthogonality blocking, discussed above). But first we look at a few other examples of systems coupled to a spin bath. 


\subsection{Some Other Systems Coupled To Spin Baths}

The previous section attempted to explain the details of a particular truncation procedure in some pedagogical detail. However there are other important physical examples of systems coupled to spin baths, which can be profitably studied if you wish to understand the truncation procedure better. I shall briefly recount how two such systems work, and then mention some other examples which can be found in the literature. The first example involves a Josephson superconducting ring, coupled to nuclear and paramagnetic spins; and the second involves a magnetic soliton (a domain wall) coupled to nuclear spins.

\section{3(a) MQC in SUPERCONDUCTORS, and the SPIN BATH}

Let us start with a system which, in the absence of the spin bath, is thought to truncate at low energies to a 2-level system, just like the giant spin system. I am thinking here of a superconducting "ring" with a single Josephson "weak link"; a typical geometry is shown in Fig.3.

FIG. 3: The geometry chosen for the analysis of the Josephson ring. Topologically, the system is a torus, with the weak link allowing the passage of a fluxon between the inside and the outside of the ring, if the current through the weak link exceeds $I_{c}$. The geometry is discussed in the text.

Following the work of a number of authors, it is understood that the flux $\Phi$ passing through the ring moves in an adiabatic ("high-energy") potential $V(\Phi)$ of form

$$
V(\Phi)=\frac{1}{2 L}\left(\Phi-\Phi_{e}\right)^{2}-2 \pi E_{J} \cos \left(2 \pi \Phi / \Phi_{o}\right)
$$

where $\Phi_{o}$ is the flux quantum $h / 2 e, L$ is the ring inductance, $\Phi_{e}$ is the external imposed flux, $\Phi$ the total flux (both of these through the ring) and $E_{J}=I_{c} \Phi_{o} / 2 \pi$ is the weak link "Josephson coupling energy", with $I_{c}$ the critical current through the ring. This "RF SQUID", and the possibility of macroscopic tunneling of $\Phi$ through the Josephson cosine 
potential, has been discussed in great detail in the literature [13, 41] (and see also Leggett's chapter in this volume). There is also a kinetic term $T=\frac{1}{2} C \dot{\Phi}^{2}$ in the system Hamiltonian; and as a result at low energies it finds itself near the bottom of a potential well, able both to oscillate (small oscillation frequency $\Omega_{o} \sim 2 \pi\left(E_{j} / \pi C\right)^{1 / 2} / \Phi_{o}$ ) or to tunnel to the nearest potential well. If these 2 wells are the 2 lowest in energy (with others at energies higher by $\Omega_{o}$ or more) then when $k T<\Omega_{o} / 2 \pi$, we can again model the system by a 2 -level system.

There is also a resistive coupling to normal electrons and Bogoliubov quasiparticles, which can be understood in terms of a coupling to a bath of oscillators (see next section). What we are interested in here is - what will be the coupling of the flux $\Phi$ to any spins in the ring? These may be either nuclear (of which there is a very large number) or paramagnetic spins (coming from magnetic impurities). We shall see in this and the next chapter that this question is of fundamental importance for the search for "Macroscopic Quantum Coherence" in RF SQUID rings. In what follows I give a qualitative discussion only, based on a detailed calculation by myself and Prokof'ev. 18]

Suppose to start with we consider the geometry shown in Fig. 3. We assume a cube of dimension $1 \times 1 \times 1 \mathrm{~cm}$ $(L=1 \mathrm{~cm})$ of type-I superconducting material, with London penetration depth $\lambda_{L}=5 \times 10^{-6} \mathrm{~cm}$, and a hole in the center of radius $R=0.2 \mathrm{~cm}$, and surface area $S=\pi R^{2}=0.1 \mathrm{~cm}^{2}$. The magnetic field inside the hole corresponding to a half-flux quantum is

$$
B_{o}=\frac{\pi \hbar c}{e \pi R^{2}}=2 \times 10^{-6} G .
$$

There is a slit in the cube, bridged by the wire-shaped junction, of length $l=10^{-4} \mathrm{~cm}$ and diameter $d=2 \times 10^{-5} \mathrm{~cm}$. The current density in the junction is enhanced over that elsewhere on the system surface by a factor $L / d=5 \times 10^{4}$, and correspondingly the magnetic field in the junction is as high as $B_{j u n} \sim B_{o} L / d=10^{-1} G$.

There are both nuclear spins and paramagnetic impurities in the spin bath. Consider first the nuclear spins; assuming all nuclei have spins, we find that in the bulk of the ring, within a penetration depth of the surface, there is a number

$$
N_{\text {ring }}=\left(L \times 2 \pi R \times \lambda_{L}\right) \times 10^{23} \approx 5 \times 10^{17},
$$

of nuclear spins coupling to the ring current; and in the junction itself, a number

$$
N_{\text {jun }}=\left(l \times \pi d \times \lambda_{L}\right) \times 10^{23} \approx 3 \times 10^{9} .
$$

coupling to the junction current. In the "high-temperature" limit the average nuclear polarisation is $M=\sqrt{N}$, and so the typical coupling between the junction current and the nuclear spins is $\Gamma_{\text {ring }} \sim \omega_{\text {ring }} M=2 \times 10^{-13} \mathrm{~K} \times 7 \times 10^{8} \approx$ $10^{-4} K$, where $\omega_{\text {ring }}=\mu_{n} B_{o}$, and $\mu_{n}$ is the nuclear Bohr magneton. For the junction nuclei one has a coupling $\Gamma_{\text {jun }} \sim \omega_{\text {jun }} \sqrt{N_{\text {jun }}} \approx 5 \times 10^{-4} \mathrm{~K}$, where $\omega_{\text {jun }}=\mu_{n} B_{\text {jun }}$, which is actually similar, because there is a linear increase in the magnetic field (since $B_{j u n} \sim 1 / d$ ), but a quadratic suppression in the number of spins (ie., $N_{j u n} \sim l d$ ). Notice the magnetic coupling is $\ll k T$, justifying the high- $T$ assumption. Notice also we have taken no account of a possible coupling to substrate spins (the ring is in superfluid He-4!), which would have a much larger coupling again to the current. We have also assumed perfect screening from external fields.

This coupling gives a longitudinal bias energy $\xi \sim 10^{-4}-10^{-3} \mathrm{~K}$, acting on the tunneling flux coordinate $\Phi$, which is rather bigger than any presently realistic numbers for the SQUID tunneling matrix element $\Delta_{o}$; we are again in a situation of strong degeneracy blocking. However, it also turns out that we have very strong orthogonality blocking! This is because each nuclear spin feels the dipolar fields from the other nuclear spins; in general there will be some component perpendicular to the field from the SQUID, of strength $\sim 1 G$, and associated energy $\omega_{k}^{\perp} \sim V_{N N} \sim 10^{-7} \mathrm{~K}$, where $V_{N N}$ is the nearest neighbour internuclear dipolar coupling energy. Physically, when the SQUID flips, the field on each nuclear spin hardly changes its direction, being dominated by the more slowly varying (but much stronger) nuclear dipolar field. 
We may summarize this analysis of the nuclear spin effects on the flux dynamics in the form of the effective Hamiltonian

$$
H_{\mathrm{eff}}=\Delta_{o} \hat{\tau}_{x}+\frac{1}{2} \sum_{k=1}^{N}\left(\omega_{k}^{\perp} \hat{\sigma}_{k}^{z}+\hat{\tau}_{z} \omega_{k}^{\|} \hat{\sigma}_{k}^{z}\right)
$$

where $\omega_{k}^{\|}=\omega_{\text {ring }}$ or $\omega_{\text {jun }}$ (with values $\omega_{\text {ring }} \sim 2 \times 10^{-13} K$ and $\omega_{\text {jun }} \sim 10^{-8} K$ respectively), whilst $\omega_{k}^{\perp} \sim 10^{-7} K$ as above. Thus $\omega_{k}^{\|} / \omega_{k}^{\perp} \ll 1$, which is the opposite limit considered to that for the giant spin! Notice further that both these couplings are much less than $\Delta_{o}$ ( $\xi$ is bigger than $\Delta_{o}$ only because there are so many nuclei involved). Thus the nuclear bath is no longer slaved to the central system at all! In the next chapter we will show that this allows us to map this problem to that of an oscillator bath, coupled to $\Phi$.

Consider now the effect of paramagnetic impurities, with concentration $n_{p m}$, a single-spin coupling $\omega_{p m} \sim$ $10^{3} \times \omega_{\text {ring }}$ to the current, and hence a total coupling energy $\Gamma_{p m} \sim 10^{6} n_{p m} \Gamma_{\text {ring }} \sim n_{p m} \times 100 \mathrm{~K}$. This is obviously bigger still, unless the superconductor is very pure indeed! The dynamics of these will come either from the Kondo effect (in the superconductor), or from flip-flop processes between these impurities. The former go at a rate $\sim T_{K}$, the Kondo temperature (which varies over many orders of magnitude depending on the impurity; the latter would go at a rate $\left(T_{2}^{-1}\right)_{p m} \sim 10^{9} n_{p m} \mathrm{~Hz}$, except that in pure samples these flip-flop processes will themselves be blocked by the local dipolar coupling between the impurity abd nearby nuclear spins (of strength $\sim 10^{-4} K$ ); thus this will happen once $n_{p m} \ll 10^{-3}$.

Just as for the Giant spins, to properly understand the flux dynamics we must then consider the nuclear bath dynamics, which can in principle relieve this blocking. We will deal with this in the next chapter.

\section{3(b) MAGNETIC DOMAIN WALL TUNNELING}

Let us now move to a quite different example, in which at low energies the central system does not behave at all like a 2-level system, but instead like a particle moving continuously in a one-dimensional potential. Of course we expect that many physical systems will behave like this at low energies, and many of them will couple to spin environments - but the following example is the only one which has so far been worked out in detail [28, 42].

The reason that a magnetic domain wall moves like a 1-dimensional particle in many realistic situations is that it is a membrane-like soliton whose "flexural" oscillations are hindered by the strong magnetic dipolar field, which tries to keep the wall flat. As an example we take the famous "Bloch wall", which has a Hamiltonian

$$
\begin{aligned}
\mathcal{H} & =\int d \mathbf{r}\left[J(\nabla \mathbf{m})^{2}-K_{\|} m_{z}^{2}+K_{\perp} m_{x}^{2}\right] \\
& =\int d \mathbf{r}\left[J\left((\nabla \theta)^{2}+\sin ^{2} \theta(\nabla \phi)^{2}\right)-K_{\|} \cos ^{2} \theta+K_{\perp} \cos ^{2} \phi \sin ^{2} \theta\right]
\end{aligned}
$$

representing a ferromagnet with easy axis along the $z$ axis and the $z-y$ plane being easy. In 3-dimensions, the units of $J$ are $\mathrm{J} / \mathrm{m}$ and the units of the anisotropy constants are in $\mathrm{J} / \mathrm{m}^{3}$.

The domain wall corresponding to this Hamiltonian is perpendicular to the $x$ axis, with the magnetisation rotating in the $z-y$ plane. We refer to the wall by its center, located at a position $Q$ along the $x$ axis The new frame of reference is thus $\left(x_{1}, x_{2}, x_{3}\right)=(z, y, x)$. This is represented in Fig.4 below.

The components of the magnetisation are given by:

$$
\hat{m}_{1}^{B}=C \tanh \left(\frac{x_{3}-Q(t)}{\lambda_{B}}\right)
$$




$$
\begin{aligned}
& \hat{m}_{2}^{B}=\chi\left(1-\frac{\dot{Q}^{2}(t)}{8 c_{0}^{2}}\right) \operatorname{sech}\left(\frac{x_{3}-Q(t)}{\lambda_{B}}\right) \\
& \hat{m}_{3}^{B}=C \frac{\dot{Q}(t)}{2 c_{0}} \operatorname{sech}\left(\frac{x_{3}-Q(t)}{\lambda_{B}}\right)
\end{aligned}
$$

$C= \pm 1$ is the "topological charge" of the wall and $\chi= \pm 1$ is the "chirality". The topological charge corresponds to the direction along which the wall moves under the application of an external magnetic field in a direction parallel to the easy axis, while the chirality refers to the sense of the rotation of the magnetisation inside the wall. A static Bloch wall only rotates in the easy plane. However, as soon as it moves it creates a demagnetising field which causes the spins to precess and the appearance of a component of the magnetisation out of the plane, directly proportional to the wall velocity. The precession of the spins also causes the appearance of an inertial term, the Döring mass, given by

$$
M_{w}=\frac{S_{w} M_{0}^{2}}{\gamma_{g}^{2}\left(J K_{\|}\right)^{1 / 2}}\left[\frac{1}{\left(1+K_{\perp} / K_{\|}\right)^{1 / 2}-1}\right]^{2}
$$

where $S_{w}$ is the surface area of the wall.

FIG. 4: A standard Bloch wall in a uniaxial ferromagnet, showing the direction of the electronic spins as one passes along a path perpendicular to the wall plane. The chirality is defined by the sense in which the spins turn, and the topological charge by the difference in magnetisation along the easy axis, between the 2 sides of the wall.

Now from this phenomenological picture of the wall, one can proceed to a treatment which eliminates the details of the wall profile altogether, and simply describes everything in terms of the coordinate $Q$ of the wall centre (assuming a wall held flat by the dipolar or "demagnetisation fields- in realistic situations wall curvature is small and has little effect on what follows). In this case the wall will have a kinetic energy (parametrised by the Döring mass), and there will also be potential terms. There are 2 obvious sources for these: 
(i) If defects are present in the sample, it will be energetically favourable for the magnetisation to rotate at this site since there is no associated energy cost. We will assume that the radius $R_{d}$ corresponding to the defect volume is much smaller than $\lambda_{B}$, the domain wall width. The wall thus becomes pinned by a potential of the form 42

$$
V(Q)=V_{0} \operatorname{sech}^{2}\left(Q / \lambda_{B}\right)
$$

with $V_{0}$ proportional to the volume of the defect. We further assume that there is a very small concentration of defects, so that there is only 1 important pinning center for the wall. This would correspond to an ideal experimental situation.

(ii) The application of an external magnetic field $\mathbf{H}_{e}$ in the direction of the easy axis couples to the magnetisation to give a potential term linear in $Q$.

We can then write a "bare" Hamiltonian (ie., neglecting the environment) for the wall 42 ]

$$
H_{w}=\frac{1}{2} M_{w} \dot{Q}^{2}-V(Q)-2 S_{w} \mu_{B} M_{0} H_{e} Q
$$

where we put the topological charge $C=1$ for brevity.

What now of the environment? In the literature you will find extensive discussion of the effect of magnons 37, 42] (ie., spin waves), electrons [43], and phonons [28] on the wall dynamics. However these are all oscillator baths. What we are interested in here is any spin bath effects. It turns out here that, just as for the nanomagnets, these are far more important than any oscillator bath effects. To see why this is we recall again the strength of hyperfine interactions to nuclear spins. Nuclear hyperfine effects vary enormously between magnetic systems. The weakest is in $\mathrm{Ni}$, where only $1 \%$ of the nuclei have spins, and the hyperfine coupling is only $\omega_{0}=28.35 \mathrm{MHz}(\sim 1.4 \mathrm{mK})$. On the other hand, in the case of rare earths, $\omega_{0}$ varies from 1 to $10 \mathrm{GHz}(0.05$ to $0.5 \mathrm{~K})$. In this latter case the hyperfine coupling energy to a single nucleus may be comparable to the other energy scales in the problem !

To derive an effective interaction Hamiltonian, we consider our system of ferromagnetically ordered spins to be coupled locally to $N$ nuclear spins $\mathbf{I}_{k}$ at positions $\mathbf{r}_{k}(k=1,2,3, \ldots N)$, which for a set of dilute nuclear spins (where only one isotope has a nuclear spin) will be random. The total Hamiltonian for the coupled system is then

$$
H=H_{m}+\sum_{k=1}^{N} \omega_{k} \mathbf{s}_{k} \cdot \mathbf{I}_{k}+\frac{1}{2} \sum_{k} \sum_{k^{\prime}} V_{k k^{\prime}}^{\alpha \beta} I_{k}^{\alpha} I_{k^{\prime}}^{\beta}
$$

where $H_{m}$ is the electronic Hamiltonian for the magnetisation (Eq. (42)), written in terms of the electronic spins $\mathbf{s}_{k}$ at the sites where there happen to be nuclear spins, and $\omega_{k}$ is the hyperfine coupling at $\mathbf{r}_{k} ; V_{k k^{\prime}}^{\alpha \beta}$ is the internuclear dipolar interaction, with strength $\left|V_{k k^{\prime}}^{\alpha \beta}\right| \sim 1-100 \mathrm{kHz}(0.05-0.5 \mu \mathrm{K})$. In terms of the continuum magnetisation $\mathbf{M}(\mathbf{r})$, we have

$$
H=H_{m}+\sum_{k=1}^{N} \omega_{k} \int \frac{d^{3} r}{\gamma_{g}} \delta\left(\mathbf{r}-\mathbf{r}_{k}\right)\left[M_{z}(\mathbf{r}) I_{k}^{z}+\left(M_{x}(\mathbf{r}) I_{k}^{x}+M_{y}(\mathbf{r}) I_{k}^{y}\right)\right]+\frac{1}{2} \sum_{k} \sum_{k^{\prime}} V_{k k^{\prime}}^{\alpha \beta} I_{k}^{\alpha} I_{k^{\prime}}^{\beta}
$$

I would like to draw your attention to the similarity between this equation and that quoted at the very beginning of section 2.2. We have in essence reduced our problem to the coupling of a single 1-dimensional coordinate to a bath of spins. I will not go into any further detail here as to how one can rewrite this Hamiltonian in terms of "degeneracy blocking" diagonal terms, plus non-diagonal terms operating when the domain wall moves (for which 
see the original paper [28]). Suffice it to say that the longitudinal term gives a total "hyperfine potential field" acting on the wall, of diagonal form

$$
U(Q)=\frac{\omega_{0} M_{0}}{\gamma_{g}} \sum_{k=1}^{N} \int d^{3} r \delta\left(\mathbf{r}-\mathbf{r}_{k}\right)\left(1-C \tanh \left(\frac{x_{3}-Q}{\lambda_{B}}\right)\right) I_{k}^{w}
$$

where the nuclear spins now have their axis of quantisation defined according to the local magnetisation orientation; the axis $w$ is along $\vec{M}(r)$, and so the component $I_{k}^{w}$ is diagonal. This potential has the spatial form of a random walk (Fig.5); however it also fluctuates in time (because of nuclear spin diffusion). There are of course non-diagonal terms as well, for which see Dubé and Stamp [28].

As discussed in detail in the original paper, the effect of this potential on the wall dynamics can be very large indeed, particularly for rare earth magnets.

FIG. 5: Typical form of the "random walk" potential $U(Q)$ acting on a magnetic domain wall, due to the hyperfine coupling to disordered nuclear spins. We assume that the temperature is higher than the hyperfine coupling to the nuclei. In reality this potential also fluctuates in time, because of nuclear spin diffusion.

This concludes our detailed discussion of the spin bath models, and where they come from. I should emphasize that there are other "canonical" spin bath models which can be studied (and which for the most part have not been, at least not at the time of writing). Two obvious ones are

(i) A model in which a simple oscillator is coupled to a spin bath. There are many obvious systems for which such a model applies- two of them are immediately apparent, for we can simply put either the SQUID flux or the domain wall mentioned above, into a harmonic potential. This would describe the SQUID trapped in one well, near the bottom, or a domain wall trapped deep in a pinning potential.

(ii) The "Landau-Zener plus spin bath model", in which a 2-level system, coupled to a spin bath, is also driven by some external ( $c$ - number) field. This problem is of considerable interest for experiments on quantum nanomagnets 
(where one applies an AC field to the magnets, and looks at the absorption), and it is also of theoretical interest, as an example of a dissipative landau-Zener model. The simplest such model involves the single passage of the 2-level system through resonance.

The reader can certainly think of more such examples (particularly after referring forward to the discussion of canonical oscillator bath models, which have been much more thoroughly studied- see section 2.4(c)).

\subsection{Coupling to The Oscillator Bath}

Let us now turn to that class of effective Hamiltonians $H_{\text {eff }}$ which can be written in terms of an environment of oscillators (the "oscillator bath" models). These models are not only very old (early examples include QED, as well as Tomonaga's model of 1-dimensional electrons, and early theories of both spin waves and the electron gas [44]), but also of great generality - they apply to almost any problem in which the central system is coupled to a set of delocalized modes (whether these be bosonic or fermionic, by the way). The lengthy history of this model means that I will refrain from giving a detailed treatment of truncation to its various low-energy forms - instead, I refer the reader to relevant original references or reviews. The reader is urged to consult these - the truncation is not without interesting subtleties, particularly where non-linear interactions to the environmental modes are involved.

Many physicists (and even more chemists) are suspicious of the claimed generality for the oscillator bath model for quantum environments, despite the justifications of Feynman and Vernon 12], and Caldeira and Leggett [13]. The essential claim is that at low energies, our general effective Hamiltonian (2) can very often be written as

$$
\begin{aligned}
H_{\mathrm{eff}}^{\mathrm{osc}} & =H_{o}(P, Q)+\sum_{k=1}^{N}\left[F_{k}(P, Q) x_{k}+G_{k}(P, Q) p_{k}\right] \\
& +\frac{1}{2} \sum_{k=1}^{N}\left(\frac{p_{k}^{2}}{m_{k}}+m_{k} \omega_{k}^{2} x_{k}^{2}\right),
\end{aligned}
$$

where $P, Q$ are the momentum and coordinate of interest, and the $\left\{x_{k}\right\}$ are harmonic oscillators with frequency $\omega_{k}<\Omega_{o}$, the UV cut-off. The crucial point is that the couplings $F_{k}$ and $G_{k}$ are weak $\left(\sim N^{-\frac{1}{2}}\right)$, and this justifies one in stopping at linear coupling. In most cases the oscillators represent delocalized modes of the environment, and the $N^{-\frac{1}{2}}$ factor then just comes from normalisation of their wavefunctions.

Now in fact physicists should not be surprised at the generality of (1.31), since it embodies precisely the same assumptions as conventional response function theory 45 - that the effect of the central coordinates $P, Q$, on each environmental mode, is weak $\left(\sim N^{-\frac{1}{2}}\right)$ and may be treated in 2 nd order perturbation theory. Then, by an appropriate diagonalisation to normal modes [46, 13], one may always write (1.31). ¿From this point of view it is clear that this should work for, say electrons - although electrons are fermions, the low-energy modes of the Fermi liquid are incoherent particle-hole pairs, plus collective modes 45. It also works nicely for superconductors 113, 41, 47 (although in this case one really needs 2 oscillator baths 447), and in fact oscillator bath models have been applied to a vast array of electronic excitations, ranging from Luttinger liquid quasiparticles to magnons.

In what follows I will attempt to convince you that the "oscillator bath' models really are very generally applicable to environments of delocalised modes. The discussion will be fairly informal- readers wanting rigour should go to the literature.

\section{4(a) SPIN-BOSON MODELS}


Consider the case where $H_{o}(P, Q)$ describes a system moving in a 2-well potential, at which point, as with the giant spin, one truncates down to the 2 lowest levels therein, to get the celebrated "spin-boson" model [48]:

$$
\begin{aligned}
H_{\mathrm{SB}} & =\Delta_{o} \hat{\tau}_{x}+\xi_{H} \hat{\tau}_{z}+\sum_{k=1}^{N}\left[\left(c_{k}^{\|} \hat{\tau}_{z}\right) x_{k}+\left(c_{k}^{\perp} \hat{\tau}_{-} q_{k}+H . c .\right)\right] \\
& +\frac{1}{2} \sum_{k=1}^{N}\left(\frac{p_{k}^{2}}{m_{k}}+m_{k} \omega_{k}^{2} x_{k}^{2}\right)
\end{aligned}
$$

with couplings $c_{k}^{\|}, c_{k}^{\perp} \sim O\left(N^{-\frac{1}{2}}\right)$, and $\vec{\tau}$ again describing the 2-level central tunneling system. Often the term $c_{k}^{\perp}$ is dropped, since formally $c_{k}^{\perp} / c_{k}^{\|} \sim \Delta_{o} / \Omega_{o}$; however sometimes one has $c_{k}^{\|}=0$ (eg. for symmetry reasons), and $c_{k}^{\perp}$ survives and is important 49 ].

Now at this point we could go through the same kind of exercise as before, and derive a spin-boson Hamiltonian at low energy for some model system. This has in fact been done by many authors for systems like SQUID's, or the Kondo problem, etc. However, to give some continuity to the discussion, let's stick to the giant spin model for the moment, since we have already seen how it truncates to a 2-level system in isolation. To see how it also couples to oscillators, we will consider a giant spin in an environment of phonons and electrons. Readers interested in SQUID's should go to Leggett's lectures; and there is of course a huge literature on the spin-boson model.

(i) Giant Spin coupled to Phonons: We thus go back to our giant spin model (eg. (8)), and ask how one should now incorporate the coupling of phonons and electrons to $\vec{S}$. Once we have done this, we can also ask how one reduces to a form like (51), once we truncate down to the lowest 2 levels of $\vec{S}$.

Let us start with phonons - this case is almost trivial, since the phonon representation is already an oscillator one. The coupling between $\vec{S}$ and the phonon variables $b_{q}, b_{q}^{+}$is just the standard magnetoacoustic one, which can either be understood from macroscopic considerations [50, 51], or at a microscopic level [52]. In either case, one has couplings like

$$
\mathcal{H}_{2}^{\phi} \sim \frac{\Omega_{o}}{S} \hat{S}_{x} \hat{S}_{z}\left(\frac{m_{e}}{M_{a}}\right)^{1 / 4} \sum_{\vec{q}}\left(\frac{\omega_{q}}{\Theta_{D}}\right)^{1 / 2}\left[b_{\vec{q}}+b_{\vec{q}}^{\dagger}\right],
$$

where $\mathrm{m}_{e}$ is the electron mass, $M_{a}$ the mass of the molecule, and $\Theta_{D} \sim c_{s} a^{-1}$ is the Debye temperature (with $a$ the lattice spacing in a molecular crystal of giant spins, and $c_{s}$ the sound velocity). This interaction describes a non-diagonal process in which a phonon is emitted or absorbed with a concomitant change of \pm 1 in $S_{z}$ (since $\left.S_{x}=\frac{1}{2}\left(S_{+}+S_{-}\right)\right)$. One also has diagonal terms of similar form, in which $S_{z} S_{x}$ is replaced by, eg. $S_{z}^{2}$; and there are also higher couplings to, eg, pairs of phonons. [50]. The bare phonon Hamiltonian is just the usual form

$$
H_{\phi}=\sum_{q} \omega_{q}\left(b_{q}^{+} b_{q}+\frac{1}{2}\right)
$$

and can be written in the usual oscillator form with the transformation $x_{q}=\left(2 m_{q} \omega_{q}\right)^{-1 / 2}\left[b_{q}^{+}+b_{-q}\right]$. Armed with (1.33) and (1.34), one can discuss the dynamics of a tunneling giant spin coupled to phonons, as has been done recently by the Grenoble-Firenze group [53, 54. At low $\mathrm{T}\left(k T \ll \Omega_{o}\right)$ one can truncate the giant spin to a 2-level system as before. The general technique for doing this is straightforward [17, 49]. 
(ii) The "Giant Kondo" Problem: Consider now a much more interesting example of a giant spin coupled to electrons. Many situations can be envisaged, depending on whether the nanomagnet, or the matrix/substrate in which it is embedded, or both, are conducting; and a great deal of physics revolves around how the electrons move across the boundary between the nanomagnet and the background matrix. Some of the this is discussed in ref. [17] (but a lot more is not!).

Here we take a simplified example which has the virtue of bringing out the essential physics. We assume that an electronic fluid freely permeates both the conducting nanomagnet (of volume $V_{o}$ ), and the background matrix; and we assume that the individual electronic spins couple to the mobile electrons via a Kondo exchange, so that at the giant spin level we have

$$
H=H_{o}(\vec{S})+\sum_{\vec{k}, \sigma} \epsilon_{k} c_{\vec{k} \sigma}^{+} c_{\vec{k} \sigma}+\frac{1}{2} \sum_{i \in V_{o}} J_{i} \overrightarrow{\hat{s}}_{i} \cdot \overrightarrow{\hat{\sigma}}^{\alpha \beta} \sum_{\vec{k} \vec{q}} e^{i \vec{q} \cdot \vec{r}_{j}} c_{\vec{k}+\vec{q}, \alpha}^{+} c_{\vec{k} \beta}
$$

where $H_{o}(\vec{S})$ is the usual Giant spin Hamiltonian, whereas $\vec{s}_{i}$ is an individual electronic spin at site $i$, and position $\vec{r}_{i}$ (cf. eq. (10)); $c_{k \sigma}^{+}$creates a conduction electron in momentum state $\mid \vec{k}>$, with spin projection $\sigma= \pm 1$ along $\overrightarrow{\hat{z}}$; and $\vec{\sigma}^{\alpha \beta}$ is a Pauli matrix. I have written this interaction in terms of the individual electronic spins in order to show its microscopic origin as an exchange interaction; but of course the locking together of all the $\vec{s}_{i}$ into a giant $\vec{S}$ means that we can immediately rewrite the interaction as

$$
H_{\text {int }}^{G K}=\frac{1}{2} \bar{J} \underline{\hat{S}} \cdot \underline{\hat{\sigma}}^{\alpha \beta} \sum_{\vec{k} \vec{q}} F_{q} c_{\vec{k}+\vec{q}_{i} \alpha}^{+} c_{\vec{k}_{i} \beta}
$$

where $\bar{J}$ is the mean value of the $J_{i}$, and $F_{q}=\int\left(d^{3} r / V_{o}\right) \rho(\vec{r}) e^{i \vec{q} \cdot \vec{r}}$ is a "form factor" which integrates the number density $\rho(\vec{r})$ of electronic spins over the volume $V_{o}$ of the nanomagnet.

This form of the coupling is very famous in condensed matter physics - it is a "Kondo coupling", but instead of being between a single spin- $1 / 2$ and conduction electrons, it couples a spin $\vec{S}$ (with $2 S+1$ levels) to the electrons. It is an unusual form of what has come to be known as the "multi-channel Kondo problem". In the energy regime over which the giant spin description is valid, one may use the "Giant Kondo" interaction in (55). At low $T$, a truncation to 2 levels produces the simple "spin-boson" Hamiltonian, with only the diagonal coupling $\hat{\tau}_{z} c_{k} x_{k}$, and with a Caldeira-Leggett spectral function

$$
\begin{gathered}
J(\omega)=\pi \alpha_{\kappa} \omega \\
\alpha_{\kappa} \sim 2 g^{2} S^{2} \int_{V_{o}} \frac{d^{3} r d^{3} r^{\prime}}{V_{o}^{2}}\left(\frac{\sin k_{F}|\underline{r}-\underline{r} \prime|}{k_{F}\left|r-r^{\prime}\right|}\right)^{2} \sim g^{2} S^{4 / 3}
\end{gathered}
$$

where $k_{F}$ is the Fermi momentum, $g=\bar{J} N(0)$ is the dimensionless Kondo coupling, and $N(0)$ the Fermi surface density of states; typically $g \sim 0.1$, so that $\alpha_{\kappa}$ is not necessarily small. Consider, eg., a very small FM particle made from $F e$, embedded in a conducting film or similar substrate/background matrix. Suppose it is only 15 Angstroms across; such a particle could still have a spin $S \sim 300$. If the conductor were, eg., $C u$, then with a $g \sim 0.1$, we would have $\alpha_{\kappa} \sim 20$. As we will see in Chapter 3 , the dynamics of this system would then be not only overdamped but frozen, in the quantum regime.

The reason for this remarkably simple effective Hamiltonian is that the number of microscopic electronic spins $\vec{s}_{i}$ in the nanomagnet $\sim O(S)$, whereas the number of interaction channels (essentially angular momentum channels for electrons scattering off $\vec{S}$ ) is $\sim S^{2 / 3}$ (proportional to the cross-sectional area of the nanomagnet), which 
means that the effective coupling per microscopic electronic spin is weak $\left(\sim S^{-1 / 3}\right)$. A renormalization group analysis of the problem substantiates this result 17 .

We see that, as previously advertised, a coupling to fermionic electrons has now been reduced to an effective coupling to bosonic oscillators. Why this happens can already be guessed from the bilinear form of the fermionic terms in (55). To an incoherent particle-hole superposition $\sum_{k, \sigma} c_{k+q, \sigma}^{+} c_{k \sigma}$ we associate a bosonic operator $b_{q}^{+}$. If we treat the coupling perturbatively (and we have just seen we can) then it is obvious that using the same transformation between $b_{q}^{+}, b_{q}$, and $x_{q}$ as we need for the phonons, we can go immediately to the oscillator bath representation. This perturbative argument is of course just a special case of the general arguments given by Feynman and Vernon [12] and Caldeira \& Leggett [13]. In the case of a stronger coupling (as in the single spin Kondo problem) the argument can actually be made in a modified form [48] (and likewise in the context of the quantum diffusion of defects in solids [55]).

¿From these examples it will perhaps be clear that a very large number of physical systems can be mapped, at low T, to a spin-boson model. Essentially all one requires is that the central system of interest may be truncated to a 2-level system, and that it be coupled to a set of extended modes. We also need to have some good reason for ignoring any couplings to a spin bath (as we shall discuss below, it is not so easy to ignore environmental spins, so that such reasons may be hard to find!). Thus, in the sense described at the beginning of this section, the spin-boson Hamiltonian constitutes an important example of a "universality class" of low-energy Hamiltonians.

\section{4(b) TWO SPINS- The "PISCES" MODEL}

I think it is worthwhile dwelling a bit on another such universality class, which also involves an oscillator bath environment. This one involves not just one but two spins, each coupled to an oscillator bath. The effective Hamiltonian is then in general of the form

$$
H=H\left(\boldsymbol{\tau}_{1}, \boldsymbol{\tau}_{2}\right)+H_{o s c}\left(\left\{\mathbf{x}_{\mathbf{k}}\right\}\right)+H_{\text {int }}\left(\boldsymbol{\tau}_{1}, \boldsymbol{\tau}_{2} ;\left\{\mathbf{x}_{\mathbf{k}}\right\}\right)
$$

where $\boldsymbol{\tau}_{1}$ and $\boldsymbol{\tau}_{2}$ are 2 Pauli spins and the $\mathbf{x}_{\mathbf{k}}$, with $\mathbf{k}=1,2, \ldots N$, are the oscillator coordinates. This model has been studied in great detail by M. Dubé and myself [57]; it is interesting in a wide variety of physical situations, particularly at the microscopic level (examples include, eg., coupled Anderson or Kondo spins, coupled tunneling defects, coupled chromophores in biological molecules, 2-level atoms coupled through the EM field, or nucleons coupled via the meson

field). Perhaps even more interesting are the applications at the macroscopic level, where $\vec{\tau}_{1}$ and $\vec{\tau}_{2}$ represent the 2 lowest levels of a pair of mesoscopic or macroscopic quantum systems. Some concrete examples include 2 coupled SQUID's, or 2 coupled nanomagnets [58]. I should add that the initial development of the model was really directed towards the understanding of quantum measurements. In this case one of the spins represents a measured system, and the other the low-energy subspace of the measuring apparatus degrees of freedom - we shall see in a minute how this can happen.

We shall be interested here in situations where $\vec{\tau}_{1}$ and $\vec{\tau}_{2}$ represent mesoscopic and/or macroscopic systems. In this case, our low-energy Hamiltonian will be as above, with the restricted form

$$
\begin{gathered}
H_{0}=-\frac{1}{2}\left(\Delta_{1} \hat{\tau}_{1}^{x}+\Delta_{2} \hat{\tau}_{2}^{x}\right)+\frac{1}{2} K_{z z} \hat{\tau}_{1}^{z} \hat{\tau}_{2}^{z} \\
H_{\text {osc }}=\frac{1}{2} \sum_{\mathbf{k}=1}^{N} m_{\mathbf{k}}\left(\dot{\mathbf{x}}_{\mathbf{k}}^{2}+\omega_{\mathbf{k}}^{2} \mathbf{x}_{\mathbf{k}}{ }^{2}\right)
\end{gathered}
$$




$$
H_{i n t}=\frac{1}{2} \sum_{\mathbf{k}=1}^{N}\left(c_{\mathbf{k}}^{(1)} e^{i \mathbf{k} \cdot \mathbf{R}_{1}} \hat{\tau}_{1}^{z}+c_{\mathbf{k}}^{(2)} e^{i \mathbf{k} \cdot \mathbf{R}_{2}} \hat{\tau}_{2}^{z}\right) \mathbf{x}_{\mathbf{k}}
$$

where the $\mathrm{N}$ oscillator bath modes $\left\{\mathbf{x}_{\mathbf{k}}\right\}$ are assumed for simplicity (but without real loss of generality) to be momentum eigenstates, and $\vec{R}_{1}$ and $\vec{R}_{2}$ are the "positions" of the two spins. The two spins are coupled through a direct interaction term $K_{z z}$ having ferro- or antiferromagnetic form, depending on whether $K_{z z}$ is negative or positive. The direct interaction itself comes from integrating out very high energy modes (with frequencies even greater than the UV cut-off $\Omega_{0}$ implicit in (61); for frequencies much lower than $\Omega_{0}$ it can be considered to be static. In the absence of the coupling to the environmental sea, the spins have their levels split by "tunneling" matrix elements $\Delta_{1}$ and $\Delta_{2}$. We work in the basis in which $|\uparrow\rangle$ and $|\downarrow\rangle$, eigenstates of $\hat{\tau}^{z}$, are degenerate until split by the off-diagonal tunneling.

We refer to Hamiltonians like this as "PISCES" Hamiltonians, where PISCES is an abbreviation for "Pair of Interacting Spins Coupled to an Environmental Sea". $H_{P I S C E S}$ as written above is clearly not the most general model of this kind. A much wider range of direct couplings is possible; instead of $K_{z z} \hat{\tau}_{1}^{z} \hat{\tau}_{2}^{z}$ we could use

$$
H_{i n t}^{d i r}=\frac{1}{2} \sum_{\mu \nu} K_{\mu \nu} \hat{\tau}_{1}^{\mu} \hat{\tau}_{2}^{\nu}
$$

We could also use more complicated indirect couplings (ie., couplings to the bath) like $\frac{1}{2} \hat{\tau}_{\alpha}^{\mu} \sum_{\mathbf{k}} c_{\mathbf{k} \mu}^{(\alpha)} e^{i \mathbf{k} \cdot \mathbf{R}_{\alpha}} \mathbf{X}_{\mathbf{k}}$, with $\mu=x, y, z$ and $\alpha=1,2$. In the present discussion we will stick to the diagonal coupling in (61) and keep only the direct coupling in $\hat{\tau}_{1}^{z} \hat{\tau}_{2}^{z}$. Our reasoning is as follows. Just as for the single spin-boson problem, we expect diagonal couplings to dominate the low-energy dynamics of the combined system, since the spins spend almost all their time in a diagonal state (only a fraction $\Delta / \Omega_{0}$ of their time is in a non-diagonal state, when the relevant system is tunneling under the barrier). In certain cases the diagonal couplings can be zero (usually for symmetry reasons), and then one must include non-diagonal couplings like $\frac{1}{2} \hat{\tau}_{\alpha}^{\perp} \sum_{\mathbf{k}}\left(c_{\mathbf{k}}^{\alpha} e^{i \mathbf{k} \cdot \mathbf{R}_{\alpha}} \mathbf{x}_{\mathbf{k}}+\right.$ H.c. $)$.

Our reason for having a direct coupling in (62) of longitudinal form is then connected to our choice of diagonal couplings between the bath and the systems. In fact, one may quite generally observe that in a field-theoretical context, any direct longitudinal interaction $K_{z z} \hat{\tau}_{1}^{z} \hat{\tau}_{2}^{z}$ can be viewed as the result of a high-energy coupling of $\hat{\tau}_{1}^{z}$ and $\hat{\tau}_{2}^{z}$ to the high-frequency or "fast" modes of some dynamic field; "fast" in this context simply means much faster than the low-energy scales of interest, so that the interaction may be treated as quasi-instantaneous. In our case, integrating out these fast modes (those with a frequency greater than $\Omega_{0}$ ) then produces the longitudinal static interaction in $H_{0}$. In the next section we see how at the frequencies of interest for the application of $H_{P I S C E S}$, a further longitudinal interaction appears, mediated by those bath modes with frequencies $\omega$ such that $\Omega_{0}>\omega \gg \Delta_{1}, \Delta_{2}$.

One further term we have omitted from (61) is an applied bias acting on one or both of the spins, of the form $H_{e x t}=\xi_{j} \hat{\tau}_{j}^{z}$, for example. We will analyse its effects in a moment; its origin is usually fairly obvious.

The crucial new ingredient, of course, in $H_{P I S C E S}$ is that we are now interested in the correlations between the behaviour of 2 systems (microscopic or macroscopic) whilst they are both influenced by the environment; even if $K_{z z}$ is zero, there will be correlations transmitted via the bath, of a dynamic and partly dissipative nature. In the next section we will see how such physics comes out of the solution for the dynamics of $H_{P I S C E S}$.

In this section we shall stick firmly to the question of the physical origin of our models. It is then useful to mention 2 concrete examples of $H_{P I S C E S}$, where $\vec{\tau}_{1}$ and $\vec{\tau}_{2}$ represent macroscopic systems. The first is a simple generalization of the "Giant Kondo" model just discussed above - we imagine 2 nanomagnetic particles in a conducting background. The second involves the much more general "measurement problem", in which one spin represents the 
low-energy Hilbert space of some quantum system, and the other the low-energy Hilbert space of a measuring apparatus; both are coupled to a quantum environment of oscillators.

(i) Two Interacting Giant Kondo Spins: We imagine starting from a high-energy Hamiltonian analogous to that for the single Giant Kondo system, now with two nanomagnets coupled to the same conducting "substrate", ie., of form

$$
H=H_{o}\left(\vec{S}_{1}, \vec{S}_{2}\right)+H_{b a t h}+H_{i n t}
$$

where $H=\left(H_{o}\left(\vec{S}_{1}\right)+H_{o}\left(\vec{S}_{2}\right)+H_{\text {dip }}\left(\vec{S}_{1}, \vec{S}_{2}\right)\right.$, describing 2 separate giant spins, coupled by a high-energy dipolar interaction; where $H_{b a t h}=\sum_{\vec{k}, \sigma} \epsilon_{k} c_{k, \sigma}^{+} c_{k, \sigma}$ as for the giant Kondo model; and finally

$$
H_{\text {int }}=\frac{1}{2} \sum_{\vec{k}, \vec{q}} \hat{\vec{\sigma}}^{\alpha \beta} \cdot\left(\bar{J}_{1} \vec{S}_{1} F_{\vec{q}}^{(1)}+\bar{J}_{2} \vec{S}_{2} F_{\vec{q}}^{(2)}\right) c_{k+q, \alpha}^{+} c_{k \beta}
$$

is the obvious generalization of the single giant Kondo coupling to the electron bath.

FIG. 6: How the problem of coupled Kondo impurities might arise in practise. We imagine 2 conducting nanomagnets (perhaps part of an array) embedded in a conducting or even semiconducting substrate. Electrons can pass across the boundary between the nanomagnets and the substrate. The nanomagnets are a distance $R$ apart, and have radius $R_{o}$.

The messy details of the truncation of this Hamiltonian to the PISCES form are given in the original paper. The main point I wish to emphasize here is the way in which the truncation generates an indirect interaction between the 2 nanomagnets, of form $\epsilon(R) \hat{\tau}_{z}^{(1)} \hat{\tau}_{z}^{(2)}$, where $R$ is the distance between the centres of the nanomagnets, and $\epsilon(R)$ is an indirect interaction which is calculated to be

$$
\epsilon(R) \sim 2 g_{1} g_{2} S_{1} S_{2} \int \frac{d^{3} r}{V_{1}} \int \frac{d^{3} r^{\prime}}{V_{2}}\left(\frac{\sin _{F}|\underline{r}-\underline{r}|}{k_{F}\left|r-r^{\prime}\right|}\right)^{2}
$$


which, not surprisingly, has the usual RKKY form; for 2 identical nanomagnets one finds [58]

$$
\epsilon(R) \sim 144 \pi^{3} \varepsilon_{F} g^{2} S^{2} \frac{\cos \left(2 k_{F} R\right)}{\left(2 k_{F} R\right)^{2}}\left[\frac{\cos \left(2 k_{F} R_{o}\right)-\sin \left(2 k_{F} R_{o}\right)}{\left(2 k_{F} R_{o}\right)^{2}}\right]
$$

where $\varepsilon_{F}$ is the Fermi energy of the substrate (ie., the bandwidth, in this simple calculation). As remarked above, the generation of such a bath-mediated coupling is quite typical. In this case the relative strengths of the dipolar and RKKY couplings depends very much on the distance between the particles, as well as the electron density- we will discuss numbers in more detail in Chapter 3.

Another example of the PISCES model is provided by a pair of SQUID rings - in this case we generate an interaction (non-Ohmic) between the rings once we integrate out high-frequency EM field modes. This is of course nothing but a fancy way of deriving the usual low-frequency inductive coupling between them.

\section{(ii) Model for Quantum Measurements:}

This brings us to a 2nd example, directly connected to the quantum theory of measurement. The standard set-up in a quantum measurement involves some "measuring coordinate" (necessarily macroscopic), and a quantum system which may be microscopic or very rarely macroscopic, which is being measured. Both are in general coupled to their surrounding environment. Now of course there are many different ways in which this set-up can be realised, but the essential features are already revealed in a model in which the quantum "system" of interest exists in one of 2 interesting quantum states, and the apparatus does the same. In this case the apparatus works as a measuring device if (and only if) its final state is correlated with the initial state of the system.

Quite how this works has been discussed qualitatively for a few models, which tend to fall in one or another of 2 classes. In the first class, the apparatus coordinate of interest can exist in one or other of 2 stable (or metastable) states, which are "classically distinguishable". The best-known example of this is the Stern-Gerlach experiment (where atomic beams are widely separated according to their microscopic spin state). Similar examples are provided by SQUID magnetometers, and one may imagine a large number of "Yes/No" experiments, in which the measuring system ends up with the relevant collective coordinate (the "measuring coordinate") in one of the 2 quite different states. By "quite different", one usually means that (a) the overlap between the 2 quantum states is very small, and (b) that the classical counterparts of the quantum states are sufficiently different that one is justified in calling them "macroscopically distinguishable". Thus, in the case of Stern-Gerlach experiment, the beams are sufficiently widely separated that they no longer overlap, and they can be effectively separated by, eg., a microscope when they hit a screen or counter. In the case of a SQUID magnetometer, the 2 relevant states differ by a single flux quantum through the SQUID ring - the overlap between the states is via tunneling, and hence exponentially small, and the currents flowing in the 2 states can differ by values $\sim \mathrm{mA}$.

A second class of models is typified by the Geiger counter. In this case we again have a "Yes/No" measuring system, designed to discriminate between 2 states of some central system under investigation. In this case the central system is typically subatomic, involving, eg., nuclear decay which has/has not occured. However the measuring system clearly involves a measuring coordinate which is not microscopic - the Geiger counter is metastable, and designed so that a large potential change $(\mathrm{kV})$ occurs between the electrodes if a microscopic (subnuclear) decay product interacts with atoms inside the counter. As has been repeatedly stressed in the literature [], this example well illustrates the massive irreversibility inherent in many measuring systems. From this point of view any further coupling of the Geiger counter to another macroscopic system (eg., to Schrodinger's cat) seems almost superfluous as far as the measurement paradox is concerned - we are already at the classical level!

The important difference between these 2 sorts of measurement is that in the latter case there is a large energy 
difference between the initial and final states of the apparatus coordinate (the energy having been fed irreversibly into an "environment" of other coordinates).

However, with our experience of the truncation procedure fresh in mind, we might legitimately ask whether it is possible to strip these coupled models down to a truncated form in which only 4 states are involved ( 2 for the central system, 2 for the apparatus). All irreversible effects arise from a coupling of these states to an environmental bath - if this is modelled by oscillators then we are back to the PISCES model.

This is part of the rationale behind recent work by M. Dubé, E. Mueller, and myself [59]. One aim of this work was to see how valid are some of the models used by quantum measurement theorists, by showing how these models may be derived from underlying "high energy" microscopic theory (and finding corrections to them). We shall see that the results have interesting consequences for measurement theory.

Before describing the work, let us first see what sort of "measurement model" we are aiming at. One very commonly used model (known as the "Bell/Coleman-Hepp" model [60]) takes the form

$$
H_{o}^{(A)}(\vec{\tau})+H_{o}^{(s)}(\vec{\sigma})+\frac{1}{2} K \hat{\tau}_{x}\left(1-\hat{\sigma}_{z}\right)
$$

where $\vec{\tau}$ is a 2-level system referring to the apparatus, and $\vec{\sigma}$ a 2-level system referring to the system. The coupling is such that if $\vec{\sigma}$ is in initial state $\mid \uparrow>$, then there is no effect on $\vec{\tau}$, whereas if $\vec{\sigma}$ is initially $|\downarrow\rangle$, then transitions can occur in the apparatus (with no change in the system state). For this measurement scheme to function properly, one requires the initial state of $\vec{\tau}$ to be $\mid \Uparrow>$ or $\mid \Downarrow>$. Typically $K$ will depend on time (ie., we turn the coupling on and then off), but this is not crucial for what follows. If we let $K=\pi$, then we have the classic "ideal measurement" scheme [], for which

$$
\begin{aligned}
& |\Uparrow>| \uparrow>\longrightarrow|\Uparrow>| \uparrow> \\
& |\Uparrow>| \downarrow>\longrightarrow|\Downarrow>| \downarrow>
\end{aligned}
$$

ie., the final state of the apparatus is uniquely correlated with the initial state of the system.

Now in view of our earlier discussion of universality classes and fixed points for low-energy Hamiltonians, it is clear that such a scheme can be obtained starting with a large variety of initial high-energy Hamiltonians. It is rather illuminating to consider one such starting point- we begin from a Hamiltonian

$$
\begin{array}{r}
H=\frac{1}{2}\left(M_{A} q_{A}^{2}+M_{s} q_{s}^{2}\right)+V_{A}\left(q_{A}\right)+V_{s}\left(q_{s}\right)-\xi_{A} q_{A} \\
-K_{o} q_{s} q_{A}+\frac{1}{2} \sum_{k=1}^{N} m_{k}\left(\dot{x}_{k}^{2}+\omega_{k}^{2} x_{k}^{2}\right)+\sum_{k=1}^{N}\left(c_{k}^{(s)} q_{s}+c_{k}^{(A)} q_{A}\right) x_{k}
\end{array}
$$

It is useful to visualize the "2-dimensional" potential here, in the space of the coordinates $q_{s}, q_{A}$ (referring to system and apparatus); see below. We assume potentials $V_{s}\left(q_{s}\right)$ and $V_{A}\left(q_{A}\right)$ which describe symmetric 2-well systems, with minima at $q_{s}= \pm 1, q_{A}= \pm 1$; however we also assume that the action required for the tunneling between the 2 apparatus states is considerably smaller than for the tunnelling between the 2 system states. The coupling $-K_{o} q_{A} q_{s}$ is a "ferromagnetic" coupling, of quite generic form (produced by expanding the interaction in powers of $q_{A}$ and $q_{s}$ ).

When we truncate this down to low energies, we get a PISCES Hamiltonian of form

$$
H \sim \Delta_{A} \hat{\tau}_{x}+\Delta_{s} \hat{\sigma}_{x}-\xi_{A} \hat{\tau}_{z}-\mathcal{K} \tau_{z} \sigma_{z}+\frac{1}{2} \sum_{k=1}^{N}\left[m_{k}\left(\dot{x}_{k}^{2}+\omega_{k}^{2} x_{k}^{2}\right)+\left(c_{k}^{(A)} \hat{\tau}_{z}+c_{k}^{(s)} \hat{\sigma}_{k}^{z}\right) x_{k}\right]
$$


where $\mathcal{K}$ is a renormalized (but still high-energy) coupling, which includes the effect of oscillators above a UV cut-off frequency $\Omega_{o}$. We will make 2 assumptions here, viz.,

$$
\text { (i) } \Delta_{A} \gg \Delta_{s}
$$

$$
\text { (ii) } \mathcal{K}>\xi_{A} \gg \Delta_{n}, k T
$$

These 2 assumptions are embodied in Fig.7 for the potential.

FIG. 7: The effective potential (shown as a contour map, with low energies shaded) for a system of 2 coupled two-well systems, with a mutual "ferromagnetic" coupling and a bias applied to the apparatus coordinate $q_{A}$ (but not the system coordinate $q_{s}$ ). On truncation to low energies this produces the biased PISCES model of eqtn. (61).

That $\Delta_{A} \gg \Delta_{S}$ implies that the apparatus reacts quickly to any change in the system state. The purpose of $\xi_{A}$ is to hold the apparatus in state $\mid \Uparrow>$ when there is no coupling; when the renormalized coupling $\mathcal{K}$ acts, it must overcome this bias. We also want the resulting net bias between initial and final states of the apparatus to be much greater than either $\Delta_{A}$ or $k T$, so that there is no chance the apparatus, once it has made a transition, can return back to its former state.

Without doing any calculations, we can immediately see from the Figure that this system will behave like an ideal measuring device. The combined system-apparatus starts off either in $|\Uparrow \uparrow\rangle$ (and stays there) or in $|\Uparrow \downarrow\rangle$ (in which case it can tunnel inelastically to $\mid \Downarrow \downarrow>$; provided we wait long enough, it will always do this, no matter how weak is the coupling of $\vec{\tau}$ to the bath). Typically the apparatus-bath coupling is strong, so that the apparatus will relax quickly. Notice, however, that the relaxation rate must depend also on the size of the transition matrix element $\Delta_{A}$, and the strong coupling to the bath will renormalize $\Delta_{A}$ down to a considerably smaller value $\Delta_{A}^{*}$. Thus we must take care that $\Delta_{A}^{*} \gg \Delta_{s}^{*}$, after both have renormalised (and the weaker coupling of the system to the bath means that $\Delta_{s}$ will decrease much less than $\Delta_{A}$ ).

In the next Chapter we will discuss the dynamics of models like these more thoroughly. Notice already, however, one obvious remark that can be made about these models. This is that any interesting dynamics possessed 
by the system before the coupling to the apparatus is switched on, is frozen by this coupling. Thus, if the system is originally tunneling between $\mid \uparrow>$ and $|\downarrow\rangle$ at a frequency $\Delta_{s}$, the large coupling $\mathcal{K}$ blocks this completely. In this sense the measurement drastically interferes with the system dynamics. We shall see in the next section that this is a quite general feature of the PISCES model, and most likely of measurements in general. What is more, a good part of $\mathcal{K}$ comes from the oscillator bath itself (indeed $K_{o}$ already arises from truncation of even higher energy modes), and the dissipative effect of the remaining oscillators (having energy $<\Omega_{o}$ ) also strongly influences the dynamics of the system once $\mathcal{K}$ is switched on, in a way which is quite different from that produced by the oscillators when $\mathcal{K}=0$. This latter feature, discussed in detail in Dubé and Stamp 57 in the context of the PISCES model, is quite new as far as we know. We thus see that models of this kind, which include both apparatus and system (as well as the environment), have interesting light to shed on measurement theory. The PISCES model appears to be the first attempt to discuss all 3 partners in the measurement operation (system, apparatus, environment) on an equal and fully quantum-mechanical level.

FIG. 8: The relationship between the measuring apparatus, the measured coordinate(s) $Q$ of interest, and the environment (ie., all other relevant degrees of freedom), involved in a typical measurement. The disciplines of mesoscopic and nanoscopic physics often involve similar situations, in which all variables must be treated quantummechanically.

It is the experience of this author that many physicists are somewhat averse to the discussion of abstract quantum measurement problems in the context of down-to-earth questions of solid-state physics. This is unfortunate, since even if one is uninterested in the foundations of quantum mechanics, there is no question that one learns a great deal about these down-to-earth problems by simply placing them in the more general context that the measurement problem requires. This is becoming really very obvious in the field of nanophysics, where both theorists and experimentalists must deal every day with the emergence of classical properties from quantum ones, and the relationship between the quantum and classical domains. In fact with a bit of hindsight one can see the steady evolution of condensed matter theory towards an ever-closer examination of the interaction between observer (or 
probe), and a quantum system of interest, in the presence of dissipation and/or decoherence (cf Fig.8). In modern mesoscopic or nanoscopic physics there is often not a huge difference in the size of the probe and the quantum system of interest. Condensed matter physics has come along way since the early days of statistical physics, when only the equilibrium behaviour of a macroscopic system was of interest!

\section{4(c) CANONICAL OSCILLATOR BATH MODELS}

Just as for the spin bath models, we have seen that it is possible to reduce the description of a large number of systems, at low energies, to that of a few effective oscillator bath models.

In this subsection I just briefly note some of the other "canonical" oscillator bath models that have also been studied. In contrast with the more recent spin bath models, many of the following are rather old, in some cases with a distinguished history - I will refer to reviews for historical details and references.

(i) Harmonic Oscillator coupled to Oscillators : A very thorough review of this model was given a few years ago by Grabert et al [61]; we are interested in a low-energy Hamiltonian of the standard form in (50), where now

$$
H_{o}(P, Q)=\frac{1}{2}\left[P^{2} / 2 M_{o}+M_{o} \Omega_{o}^{2} Q^{2}\right]
$$

and where in most cases one simply uses the bilinear coupling $H_{\text {int }}=\sum_{k} c_{k} x_{k} Q$. In this case the Hamiltonian is obviously exactly diagonalisable, and so the dynamics of the central oscillator is exactly solvable.

There are many obvious physical examples; some were already discussed by Feynman and Vernon, since many measuring systems are based on resonant absorption by one or more oscillators. An example which has stimulated a lot of recent work is that of a gravity wave detector - this is usually a very heavy bar of sphere, made from a conducting alley, in which $\Omega_{o}$ represents the principal oscillator (phases) mode. The coupling to higher phonons, or to electron-hole pairs, is deliberately made very weak. The purpose of these detectors is to absorb a single quantum of energy in this mode, from a gravitational wave. An enormous literature exists on this kind of application; see in particular the books by Braginsky and collaborators [62], which are particularly interesting for the generality of their coverage. It has indeed been remarked on many occasions that one can understand much of Quantum Mechanics with reference to 2 simple models (the 2-level system and the harmonic oscillator); in these lectures I am simply coupling these to an environment!

One can obviously also consider systems of coupled oscillators, in analogy with the PISCES model.

(ii) Tunneling/Nucleating System, coupled to Oscillators: Another canonical model which has been studied in hundreds if not thousands of papers is that of a particle tunneling from a potential well into an open domain - usually the problem is designed so that this domain is semi-infinite, and the relevant potential is 1-dimensional. This restriction is actually rather weak, since many multi-dimensional tunneling or nucleation problems can be reduced to 1-dimensional one, as already noted in our discussion of domain wall tunneling in the spin bath. In the context of tunneling of systems coupled to oscillators, this question has been discussed in great detail by A. Schmid [63].

The relevant Hamiltonian is exactly that in (2.1), with $\mathrm{V}(\mathrm{Q})$ representing the tunneling potential - this is the famous "Caldeira-Leggett" model [13]. So many discussions have been given of how models like this can be derived, that I do not propose adding to them here.

(iii) Free Particle coupled to Oscillators. If we let $\mathrm{V}(\mathrm{Q})=0$ in $(2.1)$, we get a model which is of somewhat academic interest but which can be solved for a variety of couplings - it is particularly interesting for the study it allows of different initial conditions imposed on the environmental state, and an initial correlations between bath and particle [61]. 
This concludes our survey of the various ways in which one may truncate high-energy Hamiltonians down to one or other of the universality classes mentioned in the introduction. We now proceed to a survey of the dynamics of these models.

\section{Tunneling Dynamics: Resonance, Relaxation, Decoherence}

\subsection{Generalities}

There are a number of features common to any problem in which some central quantum system tries to tunnel (or otherwise show coherent dynamics), whilst coupled to an environment. Let us first note 2 important mathematical features. One is the division of coupling terms in $H_{\text {eff }}$ into "diagonal terms" (operating when the system is not tunneling), and "non-diagonal terms" (active during the tunneling). The difference is very clear when the central system is a 2-level one, and easily shown diagramatically, for either spin or oscillator baths. A second feature appears when one comes to average over the bath variables, to produce a reduced density matrix for the central system. Mathematically this averaging supposes some distribution of probability over the possible bath states, which is assumed invariant in time - often it is simply a thermal average, assuming a thermal equilibrium distribution for the bath states. For the time invariance to be physically reasonable, it is necessary that the energy transferred from the central system, while it is relaxing, be rapidly distributed over the bath states, via some kind of mixing. This assumption is usually reasonable for the oscillator bath (although one has to be careful sometimes about non-linear effects). For the spin bath, often containing a finite number $\mathrm{N}$ of spins (no thermodynamic limit), the assumption can be wrong, and then one has to think more carefully (see later).

All these problems also have obvious physical features in common. The environmental wave-function "entagles" with that of the central system, thereby destroying coherence in the latter; and if we start the central system in an excited state, it decays or relaxes incoherently by exciting bath modes. In a large system this may occur via tunneling into a quasi-continuum of states ("Macroscopic Quantum Tunneling"), but at low energies it is often more common to have tunneling between discrete levels on either side of a barrier. In this case, if the bias $\xi=\left(\varepsilon_{L}-\varepsilon_{R}\right)$ between these 2 levels is substantially larger than the tunneling matrix $\Delta$, resonance is lost and tunneling is suppressed; the overlap integral between $\mid L>$ and $\mid R>$ is now $\sim \Delta /\left(\Delta^{2}+\xi^{2}\right)^{\frac{1}{2}}$, and so the new effective "tunneling amplitude" is $\Delta^{2} /\left(\Delta^{2}+\xi^{2}\right)^{\frac{1}{2}}$ (compare the off-diagonal matrix elements in the density matrix of eqtn. (28), for the simple 2-level system). However at this point the bath can come to the rescue, by absorbing the energy difference $\xi$, and thereby mediate inelastic (and thus incoherent) tunneling. Other related processes of interest are quantum diffusion [55] (in which the diffusion rate of a system in the quantum regime increases as one lowers the temperature), and quantum nucleation (which is essentially barrier tunneling).

Quite generally we may characterise the dynamics of the central system via the reduced density matrix; for the general Hamiltonian (1.2) this is

$$
\rho\left(Q, Q^{\prime} ; t\right)=\operatorname{Tr}_{\left\{q_{k}\right\}}\left[\rho_{t o t}\left(Q, Q^{\prime} ;\left\{q_{k}\right\},\left\{q_{k}^{\prime}\right\} ; t\right)\right]
$$

where the trace is over the environmental variables (usually with a thermal weighting). Our problem is to evaluate the propagator $K_{12}$ for $\rho$, defined by

$$
\rho\left(Q, Q^{\prime} ; t\right)=\int d Q_{2} \int d Q_{2}^{\prime} K(1,2) \rho\left(Q_{2}, Q_{2}^{\prime} ; t_{2}\right)
$$

In this equation, $\mathrm{K}(1,2)=K\left(Q_{1}, Q_{1}^{\prime} ; Q_{2}, Q_{2}^{\prime} ; t_{1}, t_{2}\right)$, and is the 2-particle Green function describing the propagation of the central system, first from $Q_{1}$ (at time $t_{1}$ ) out of $Q_{2}$ (at time $t_{2}$ ); and then back from $Q_{2}^{\prime}$ (at 
time $t_{2}$ ) to $Q_{1}^{\prime}$ (at time $t_{1}$ ). $\mathrm{K}(1,2)$ simply describes the "flow" of the reduced density matrix in $\left(Q, Q^{\prime}\right)$ space (Fig.9). "Decoherence" in the " $Q$-space representation" then corresponds to the suppression of "off-diagonal" parts of $\rho\left(Q, Q^{\prime}\right)$, for which $Q$ and $Q^{\prime}$ differ significantly.

FIG. 9: The flow of the reduced density matrix in the space of generalised coordinate variables $\left(Q, Q^{\prime}\right)$. The propagator $K(1,2)$ then simply describes this flow. If there is strong decoherence in the coordinate variables, the suppression of off-diagonal interference then tends to compress the flow along the streamlines, as in the figure.

All of these features are true of tunneling systems, regardless of the bath. However in what follows we shall see in detail how going between a spin and oscillator bath can change some very important details.

\section{1(a) OSCILLATOR BATHS}

As noted by Feynman and Vernon [12, the oscillator bath environment is easily integrated out because the oscillator actions are quadratic. Thus the central system density matrix has a propagator

$$
K(1,2)=\int_{Q_{1}}^{Q_{2}} d Q \int_{Q_{1}^{\prime}}^{Q_{2}^{\prime}} d Q^{\prime} e^{-i / \hbar\left(S_{o}[Q]-S_{o}\left[Q^{\prime}\right]\right)} \mathcal{F}\left(Q, Q^{\prime}\right)
$$

Here $S_{o}[Q]$ is the free central system action, and $\mathcal{F}\left(Q, Q^{\prime}\right)$ is the famous "influence functional", defined in general by

$$
\mathcal{F}\left(Q, Q^{\prime}\right)=\prod_{k}\left\langle\hat{U}_{k}(Q, t) \hat{U}_{k}^{\dagger}\left(Q^{\prime}, t\right)\right\rangle,
$$

where $\hat{U}_{k}(Q, t)$ describes the evolution of the $k$-th environmental oscillator, given that central system follows the path $Q(t)$ on its "outward" voyage, and $Q^{\prime}(t)$ on its "return" voyage. Now we can always write this as

$$
\mathcal{F}\left(Q, Q^{\prime}\right)=e^{-i \Phi\left(Q, Q^{\prime}\right)}=e^{-i \sum_{k=1}^{N} \phi_{k}\left(Q, Q^{\prime}\right)},
$$


where $\Phi\left(Q, Q^{\prime}\right)$ is a complex phase, containing both real (reactive) contributions, and imaginary damping contributions. Thus $\mathcal{F}\left(Q, Q^{\prime}\right)$ acts as a weighting function, which weights different possible paths $\left(Q(t), Q^{\prime}\left(t^{\prime}\right)\right)$ differently from what would happen if there was no environment (thus, if the oscillators couple directly to the coordinate via a coupling $F_{k}(Q) x_{k}$, then $\mathcal{F}_{k}\left(Q, Q^{\prime}\right)$ will typically tend to suppress "off-diagonal" paths in which $Q$ and $Q^{\prime}$ differ strongly).

The crucial result of weak coupling to each oscillator is that an expression for each $\mathcal{F}_{k}\left(Q, Q^{\prime}\right)$ can be written down (and thence for $\mathcal{F}\left(Q, Q^{\prime}\right)=\prod_{k} \mathcal{F}_{k}\left(Q, Q^{\prime}\right)$ ), in which $\phi_{k}\left(Q, Q^{\prime}\right)$ is expanded to 2 nd order in the coupling only [12, 13]. Since the couplings are $O\left(N^{-1 / 2}\right)$, summing over the $N$ oscillators then gives an expression for $\Phi\left(Q, Q^{\prime}\right)$ which is independent of $N$ and exact in the thermodynamic limit.

FIG. 10: Graphical representation of the influence functional phase $\Phi\left(Q, Q^{\prime}\right)$. In (a) I show the 4 contributions to 2nd order in the coupling $F_{k}(Q)$; the wavy line is the propagator $g_{k}\left(t-t^{\prime}\right)$ for the $k$-th bath oscillator. Exponentiation gives graphs to all orders; a typical example is shown in (b).

The second-order (in $\left.F_{k}(Q)\right)$ graphs contributing to $\Phi\left(Q, Q^{\prime}\right)$ are shown in Fig.10(a); the exponentiation of these gives graphs like those in Fig. 10(b). The bosonic propagator for each oscillator, in equilibrium, is just

$$
g_{k}(t)=\frac{1}{2 m_{k} \omega_{k}}\left[e^{i \omega_{k} t}+2 \frac{\cos \omega_{k} t}{\exp \left\{\beta \hbar \omega_{k}\right\}-1}\right] \quad\left(\beta=1 / k_{B} T\right) .
$$

Summing over all bosonic modes allows us to subsume all environmental effects into a spectral function 12 , 13], whose form I quote here in the case where the coupling $F_{k}(Q)$ is linear in $Q$, ie., $F_{k}(Q)=c_{k} Q$ (so that the system-oscillator coupling is bilinear):

$$
J(\omega)=\frac{\pi}{2} \sum_{\kappa=1}^{N} \frac{\left|c_{\kappa}\right|^{2}}{M_{\kappa} \omega_{\kappa}} \delta\left(\omega-\omega_{\kappa}\right)
$$

which of course just has a "Fermi golden rule" form, characteristic of response functions. As emphasized by Leggett, at low $\omega$ this function will often be dominated by an "Ohmic" term $J(\omega) \sim \eta(\omega)$ (where in general $\eta$ depends on 
temperature). This is partly because of course a linear form dominates over higher powers at low $\omega$. However the reason for the common occurence of the Ohmic form is that it is linked in the classical dissipative dynamics to Ohmic friction. In this context it is important that even if one finds, in some calculation, a non-Ohmic form for $J(\omega)$, it is almost certain that if one pursues the calculation to higher orders, an Ohmic coupling will be found. Often however this coupling will usually be temperature-dependent, ie., we will have $J(\omega) \rightarrow J(\omega, T)$. For a more detailed discussion of this point, see, eg., Dubé and Stamp [28], and refs. therein.

The function $J(\omega)$ is usually called the "Caldeira-Leggett spectral function".

\subsection{Dynamics of the Spin-Boson Problem}

As our first example of the dynamics of a system coupled to an oscillator bath, we go to the famous spin-boson problem. There are several detailed reviews of the spin-boson dynamics [48, 41, 64, (see also Leggett's notes in this volume). Here I summarize results relevant to the present topic.

Although one can imagine a whole variety of forms for the spectral function $J(\omega)$ in this problem, in many systems it is the Ohmic coupling which dominates at low energies, and I will only talk about this case here. Readers interested in the finer details can go to the reviews, particularly if they are interested in non-Ohmic spin-boson systems. For the Ohmic spin-boson problem one writes $J(\omega)=\pi \alpha \omega$, and enormous effort has been devoted to understanding the phase diagram and dynamics of the central spin, in contexts ranging from particle theory, the Kondo problem, defect tunneling and quantum diffusion, flux tunneling in SQUID's, and 1-dimensional fermion problems. From these studies we recall several results. First, the well known phase diagram for Ohmic coupling 48 (Fig.11).

FIG. 11: The "phase diagram" showing the different dynamic behaviours possible for an unbiased 2-level system coupled to an Ohmic bath, as a function of the dimensionless coupling $\alpha$ to the bath, and the temperature $T$. At $T=0$ and $\alpha>1$, the bath oscillators "quantum localise" the system- it freezes. For $\alpha \gg 1$, the system is quasi-localised for temperatures up to a crossover temperature $T_{c} \sim \Omega_{o} / 2 \pi$. Elsewhere the system shows overdamped relaxation (exactly exponential along the Toulouse line $\alpha=1 / 2$ ), except for low $T$ and low $\alpha$. 
The most important feature here is how delicate quantum coherence turns out to be, as a function of the dimensionless parameters $\alpha$ and $T / \Delta_{R}$, where $\Delta_{R}(\alpha)$ is a renormalized tunneling amplitude. This is of course because (i) the oscillators suppress $\Delta_{o}$ to $\Delta_{R}$, via a standard Franck-Condon effect, and (ii) the coupling $c_{\kappa}$ to $\hat{\tau}_{z}$ allows the oscillators to sense or "measure" the state of $\hat{\tau}$, and thereby suppress coherent oscillations.

A second important result, which concerns many oscillator bath models (not just the spin-boson model), is the relationship between the classical and quantum behaviour of the system coupled to the oscillator bath. It is of course trivially obvious that if the spectral function $J(\omega ; T)$ contains all information about the system/bath coupling, then both the classical frictional dynamics and the low-T tunneling dynamics are determined by the same $J(\omega, T)$. In this sense knowledge of the classical motion may help us in predicting the quantum dynamics (albeit at a different $\mathrm{T}$ ). However in those (not uncommon) cases where $J(\omega)=\eta \omega$, with $\eta$ independent of $T$, Caldeira and Leggett showed that knowledge of $\eta$ obtained from the classical friction entirely determined the quantum dynamics.

One should, nevertheless, refrain from pushing this result too far. In particular, it is a serious mistake to imagine that one can understand the decoherence in the quantum dynamics, solely from a knowledge of the classical dynamics. A related mistake is the idea that there is some well-defined "decoherence time", referring to exponential relaxation of off-diagonal matrix elements - as a rule this is incorrect, even in the oscillator bath models.

To better appreciate the above results, a brief word on the mathematical techniques is in order. To solve for the dynamics of the spin-boson model, Leggett et al. 48 employed an approximation dubbed the "NIBA" ("NonInteracting Blip Approximation"), in order to evaluate the influence functional. This approximation simply assumes that the system density matrix (with oscillators already averaged over) spends most of its time in a diagonal state (as opposed to an off-diagonal one). It is useful to understand this approximation in 3 different ways.

FIG. 12: A typical path $Q(t)$ for a 2-state system coupled to an environment. The 2 levels result from truncating a higher-energy Hamiltonian containing many levels (here a giant spin $\vec{S}$ ). The fast high-energy fluctuations are invisible, and only the rapid (on a timescale $\Omega^{-1}$ ) transitions between the 2 classically stable states $\vec{S}_{1}$ and $\vec{S}_{2}$ are seen. The system interacts diagonally $(\mathrm{D})$ or non-diagonally (ND) with the environment; in the latter case a non-linear coupling to an environmental triplet is shown (as in the coupling to the spin bath). 
(i) diagramatically, the system is in a diagonal state (dubbed a "sojourn" by Leggett et al. [48]), when both paths contributing to the density matrix evolution happen to be in the same state. An off-diagonal (or "blip") state, where the 2 paths at a given time are in opposite states, is then considered to be rare.

(ii) mathematically, the suppression of the off-diagonal elements arises because the propagator $g_{k}(t)$, for the oscillators, is complex, and the imaginary part damps out off-diagonal blip states. Notice that by "diagonal" we mean diagonal in $\hat{\tau}_{z}$; and the environment couples to $\hat{\tau}_{z}$.

Formally we can express this point in terms of the influence functional as follows. For an arbitrary path of the system, the complex phase $\Phi\left(Q, Q^{\prime}\right)$ in $(78)$ can be written

$$
\Phi\left(Q, Q^{\prime}\right)=\int_{0}^{t} d \tau \int_{0}^{\tau} d s(i \Sigma(\tau-s) \xi(\tau) \chi(s)-\Gamma(\tau-s) \xi(\tau) \xi(s))
$$

where the double path is parametrised by sum and difference variables $\xi(s)=Q(s)-Q^{\prime}(s)$ and $\chi(s)=Q(s)+Q^{\prime}(s)$ (again we assume a coupling of the oscillators to $Q$ ). Now for the specific case of the spin-boson system system, the paths are very simple; they are nothing but sums over outgoing or return paths of the form

$$
Q_{(n)}(s)=1-\sum_{i=1}^{2 n}\left[\operatorname{sgn}\left(s-t_{2 i-1}\right)+\operatorname{sgn}\left(t_{2 i}-s\right)\right],
$$

where $\operatorname{sgn}(x)$ is the sign-function, $n$ is the number of transitions of the central system, occuring at times $t_{1}, t_{2}, \ldots, t_{2 n}$ (and here for definiteness we assume trajectories starting and ending in the same state). Notice that the paths have sudden jumps because once we truncate our Hamiltonian to energies $\ll \Omega_{o}$, we have no way of resolving processes occurring over the time scale $\Omega_{o}^{-1}$ of the instanton jump- to all intents and purposes they are instantaneous! I have shown in Fig.12 a typical such path (including the couplings to the environmental modes, which in a path integral formalism take place at particular times).

Notice that now the off-diagonal "blip" states are simply the ones having finite $\xi(s)($ and $\chi(s)=0)$. How and when are these suppressed by the oscillator bath? Well, we can see this by looking at the forms of the reactive and dissipative contributions to the phase $\Phi\left(Q, Q^{\prime}\right)$, which can easily be calculated in terms of the spectral function $J(\omega)$ for the bilinear coupling model (which just integrates over the complex propagator $g_{k}(t)$ ):

$$
\begin{gathered}
\Sigma(\tau-s)=\int_{0}^{\infty} d \omega J(\omega) \sin \omega(\tau-s) \\
\Gamma(\tau-s)=\int_{0}^{\infty} d \omega J(\omega) \cos \omega(\tau-s) \operatorname{coth}(\omega / 2 T)
\end{gathered}
$$

¿From this we see that whether blips are suppressed relative to sojourns depends very much on the form of $J(\omega)$ (for a detailed discussion see Leggett et al. 48), but if they are, it is because of the imaginary part $\Gamma$ of the complex phase.

(iii) Finally and most physically, we can see that the diagonal state of the density matrix is favoured because the environment is in effect continually "measuring" $\hat{\tau}_{z}$ (the coupling $\sum_{k} c_{k} x_{k} \hat{\tau}_{z}$ distinguishes between $\mid \uparrow>$ and $\mid \downarrow>)$; this suppresses quantum interference between the two states (ie., off-diagonal elements). Notice that we can think of this as a "dynamic localisation" of the system in one or other state for long periods, and the opportunity for tunneling is reduced to periods when resonance between the 2 wells persists long enough for tunneling to occur. Note that this is not really the same as the "degeneracy blocking" we encountered before, which comes from a static 
bias field. Nevertheless it does bring us to perhaps the most important question about the spin-boson model, which is, what happens to the spin when there is an external bias field $\xi$, and a finite temperature? I will simply quote the (practically very useful) answer for you here []. Suppose the system starts up in a state $|\uparrow\rangle$ at $t=0$. Then at time $t$ later the probability $P_{11}(t)$ that it will still be $|\uparrow\rangle$ is given by the simple incoherent relaxation expression $P_{11}(t)=e^{-t / \tau(T, \xi)}$, where for the most interesting case of Ohmic coupling, the rate $\tau^{-1}$ is given by (assuming energy scale less than $\Omega_{o}$ as usual):

$$
\tau^{-1}(\xi, T)=\frac{\Delta^{2}}{2 \Omega_{0}}\left[\frac{2 \pi T}{\Omega_{0}}\right]^{2 \alpha-1} \frac{\cosh (\xi / 2 T)}{\Gamma(2 \alpha)}|\Gamma(\alpha+i \xi / 2 \pi T)|^{2}
$$

where $\Gamma(x)$ is the Gamma function. This result is valid for all but the smallest biases, and even when the bias is $\leq \Delta$, it is valid throughout the incoherent relaxation region of the phase diagram in Fig. XXX. Thus even at very low bias we get relaxation at a rate

$$
\tau^{-1}(T)=\frac{\Delta^{2}}{2 \Omega_{0}} \frac{\Gamma^{2}(\alpha)}{\Gamma(2 \alpha)}\left[\frac{2 \pi T}{\Omega_{0}}\right]^{2 \alpha-1}+O(\xi / T)^{2} \quad(\xi / T \ll 1)
$$

whereas in the opposite limit where we apply a strong bias $\xi \gg T$ to the system, we get incoherent fluctuations between the 2 states:

$$
\tau^{-1}(\xi)=\frac{\Delta^{2}}{\Omega_{0}} \frac{1}{\Gamma(2 \alpha)}\left[\frac{\xi}{\Omega_{0}}\right]^{2 \alpha-1}
$$

These results have been applied to a large variety of problems in physics, particularly those involving conduction electron baths. Their application to SQUID dynamics has been particularly emphasized by Leggett [41, 65].

Application to the Giant Kondo model: The application to the problem of a nanomagnet coupled to a conducting bath illustrates some of the dramatic features of the above results. Consider the example given previously of an Fe particle with $S=300$ and dimensionless coupling $\alpha_{\kappa} \sim 20$ to the electronic bath. Then at zero bias, the incoherent fluctuation rate goes as the 40-th power of temperature, whereas at low $T$, the incoherent relaxation rate goes as the 40-th power of bias! Consider now an array of such particles, which we imagine to be functioning as a computer data storage bank. If we are at low $T$, and small bias, the giant spins are "quantum localised"; they only relax over eons of time (the crucial point is to stay below the crossover temperature $T_{c}=\Omega_{o} / 2 \pi$; if $T=T_{c} / 2$, the relaxation time is already $10^{6}$ years!). Thus even though these particles are no larger than small molecules, they can store information for astronomical time periods below $T_{c}$ (and recall that $T_{c}$ can easily be $1 K$ if we have a reasonably strong anisotropy!). On the other hand suppose we keep $T$ well below $T_{c}$, and raise the bias. Then over the time-window that might be of interest to data storage (ie., between $m s e c$ and years), we will see a very sudden "switching-on" of the dynamics of the giant spins, in the small region of bias around $\xi \sim \Omega_{0}$.

This result is obviously of potential practical importance (as already discussed in the original paper [17])! In this context an experimental test of these results is highly desirable. Note that one cannot of course extend these results above $T, \xi>T_{c}$, since our truncated Hamiltonian does not work there (and in fact when $T>T_{c}$, we go into the thermally activated regime). Note also that these results also do not account for the effects of the nuclear spin bath; for the combined effects of the 2 baths, see Prokof'ev and Stamp [17].

\subsection{Other Bosonic Models}

The spin-boson model reveals much about the low-energy dynamics of a system coupled to an oscillator bath - but much is obviously missing as well. In what follows I quickly review the main results for a few other canonical models. 


\section{3 (a) The PISCES MODEL}

This is the simplest non-trivial generalization of the spin-boson model. As we saw in section 2.4, what is interesting here is the communication between the spins via the bath, and the way in which each affects the dynamics (including the coherence properties) of the other.

To characterize the dynamics of the model one calculates the 2 -spin reduced density matrix $\rho\left(\tau_{1} \tau_{2} ; \tau_{1}^{\prime} \tau_{2}^{\prime} ; t\right)$, which is a $4 \times 4$ time- dependent density matrix. Instead of discussing how these calculations were done (for which see the long paper of Stamp and Dubé [57]), I think it will be more useful here to give an intuitive feeling for the results, and some of their consequences for coupled mesoscopic or macroscopic systems.

The most interesting examples of the PISCES model have Ohmic coupling of each spin to the oscillator bath (cf. section 2.3). In this case one can delineate the behaviour of each spin by a "phase diagram" in the 4-dimensional space of couplings $\alpha_{1}, \alpha_{2}, \mathcal{J}(\mathcal{R})$, and $T$, where $\alpha_{1}, \alpha_{2}$ are the Ohmic coupling of each spin to the bath, and $\mathcal{J}$ the total static coupling between the spins, including the bath-mediated coupling $\epsilon(R)$ (section 2.4). The other 2 energy scales in the problem are the $\Delta_{\beta}^{*}$, where $\beta=1,2$ labels the 2 spins, and the star superscripts indicate the renormalised tunneling matrix elements (after coupling to the bath). Then there are 4 dimensionless parameters in the problem, viz., $\Delta_{\beta}^{*} / \alpha_{\beta}, \mathcal{J} / T$, and $T$ itself; this is our parameter space.

The simplest case to discuss is where the spins are identical (ie., $\Delta_{1}=\Delta_{2}=\Delta$, and $\alpha_{1}=\alpha_{2}=\alpha$ ). The

behaviour can then be displayed in a simple way as a function of $\mathcal{J}(\vec{R}) / \alpha$ and $T$ (cf. Fig.13), and we can identify 4 different regions in this phase diagram, as follows:

FIG. 13: The phase diagram for the symmetric PISCES model (both spins identical), for an Ohmic bath, as a function of the dimensionless parameters $\ln \left(T / \Delta^{*}\right)$ and $\ln \left(\mathcal{J} / \Delta^{*}\right.$, where $\mathcal{J}$ is the full renormalised coupling between them. We assume the dimensionless coupling $\alpha$ of each to the bath is $\ll 1$, so the mutual coherence phase appears.

(i) The "Locked Phase" $\left(\mathcal{J} \gg T, \Delta_{\beta}^{*} / \alpha_{\beta}\right)$ : In this regime, the effective coupling $\mathcal{J}$ is so strong that the 2 spins lock together, in either the states $|\uparrow \uparrow\rangle$ or $|\downarrow \uparrow\rangle$ depending on the sign of $\mathcal{J}$. One finds that the combined 
"locked spin" oscillates between $|\uparrow \uparrow\rangle$ and $|\downarrow \downarrow\rangle$ (for FM coupling), or between $|\uparrow \downarrow\rangle$ and $|\downarrow \uparrow\rangle$ (for AFM coupling), at a renormalised frequency $\Delta_{c}=\Delta_{1} \Delta_{2} /|\mathcal{J}|$. This result is not at all surprising- in fact one would get the same result just by coupling 2 tunneling spins in the complete absence of the environment! However the oscillations are now damped - in fact the locked spin now behaves like a single spin-boson system, with a new coupling $\alpha_{c}=\alpha_{1}+\alpha_{2} \pm 2 \alpha_{12}$ to the oscillator bath, the $+(-)$ corresponding to ferromagnetic (antiferromagnetic) coupling between the spins. For the dynamics of the locked spin one simply then refers back to the spin-boson results.

(ii) The "Mutual Coherence" phase $\left(\Delta_{\beta} / \alpha_{\beta} \gg T \gg \mathcal{J} ; \mathcal{J}>\Delta_{\beta}\right)$ : Here, the thermal energy overcomes the mutual coupling; nevertheless if the dissipative couplings $\alpha_{\beta}$ 's are sufficiently small $\left(\alpha_{\beta} \ll 1\right)$, it is possible for the energy scale $\Delta_{\beta}^{*} / \alpha_{\beta}$ to dominate even if $\Delta_{\beta}<\mathcal{J}$. In this case, even though we are dealing with a strong coupling, and the bath dissipation is still important, some coherence in the motion of each spin is maintained - moreover, the small $\mathcal{J}$ causes "mutual coherence" between the two spins, ie., their damped oscillations are correlated to some extent. The analytic form of the correlation functions is extremely complex (see the original paper [57), and moreover is not given simply in terms of the single spin-boson results; the mutual correlations are essential in this regime. However the physics is fairly easy to understand- we are seeing a kind of "beat" or "breather" oscillation between the 2 spins, which the damping has not quite succeeded in destroying. In addition, each spin is exhibiting its own weakly damped oscillations. The situation is reminiscent of 2 guitar strings, which couple through the air (and the guitar box), but where the air also causes weak damping.

FIG. 14: The Probability $P_{\tau_{1}^{z} \tau_{2}^{z}}(t)$ for the 2 PISCES spins to be in state $\left|\tau_{1}^{z} \tau_{2}^{z}\right\rangle$ after a time $t$, if at $t=0$ they started in state $|\uparrow \uparrow\rangle$. The system is in the "Correlated relaxation" regime, with time shown logarithmically, in units of inverse temperature. We assume $\alpha_{1}=1.5, \alpha_{2}=2, T / \Delta_{1}=100, T / \Delta_{2}=300$, and a ferromagnetic coupling $\mathcal{J} / T=-0.04$.

(iii) The "Correlated Relaxation" or High-T phase $\left(T \gg \Delta_{\beta} / \alpha_{\beta}, \mathcal{J}\right)$. In this regime, the bath causes each spin to relax incoherently; however, the relaxation of the two spins is still correlated (indeed each spin relaxes in the time-dependent bias generated by the other). This regime is, along with the locked regime, the most typical. The 
coupling between the 2 spins, via the bath oscillators, simply decoheres completely the spin dynamics of each, and they are both strongly damped. You can find the analytic expressions for their damping in the original paper []; what is perhaps remarkable is that the system exhibits 3 different damping rates, each of which is however a fairly simple algebraic function of the damping rates for 2 different single spins, each coupled to an oscillator bath and in a static bias field $\xi=\mathcal{J}$ (cf. equation (85p). In this sense the problem is not fundamentally different from the single spin-boson model. Here I just show a typical relaxation of the 4 diagonal elements of the 2 -spin density matrix, which illustrates the vastly different relaxation timescales which can result during the relaxation of 2 initially parallel spins (Fig.14).

(iv) Finally, and much less interesting, the "perturbative regime" $\left(\mathcal{J} \ll \Delta_{\beta}^{*}\right)$, in which the total coupling is so weak that the 2 spins relax almost independently; all correlations can be handled perturbatively, and only weakly affect the behaviour that one calculates from the standard spin-boson model.

The remarkable thing is that analytic results for the dynamics of the 2 spins can be found, although we shall not need these in this lightning survey. Readers familiar with some particular case of the PISCES model will doubtless recognise features of the phase diagram. Thus, eg., those who have looked at the problem of 2 coupled Kondo impurities will recognise the "locked regime" from the old studies of Wilkins et al [66]. The advantage here is that instead of just extracting a phase diagram (which is easily done using renormalisation group methods), we have been able, using instanton methods, to extract the dynamic properties as well.

Perhaps the most important thing to take away from these results is just how easily the quantum dynamics of each spin can be decohered or even blocked by the other. We should not be too surprised by this - recall how the environment causes a "dynamical localisation" of the spin in the single spin-boson model. However it is much more serious in the PISCES model, because to the weak random environmental bias we have added a much stronger dynamical bias coming from the other spin. From this point of view it is actually interesting to compare the PISCES dynamics with that of a much simpler "toy model", in which the environment is eliminated completely, and we just have 2 coupled spins (see Dubé and Stamp [57]).

Let us now fill out this picture by applying it to the 2 physical cases discussed in section 2.4.

(i) 2 coupled Giant Kondo spins: As mentioned in the previous sub-sections, the dynamics of a Giant Kondo spin is of potential technological importance. However it is also obvious that in a nanomagnetic array, interactions between the nanomagnets can alter the behaviour of a single nanomagnet. Since there is obviously a large number of different cases that can be discussed [17, I will simply give you a taste of the results. Consider again the example in which a Fe-based nanomagnet is embedded in a conducting film, whose conduction electron density can be varied. We again assume $S=300$, and now also assume $\Omega_{o}=1 K, \Delta=1 \mathrm{mK}$, and a typical nuclear bias energy $E_{o} \sim 6 m K$ (roughly 2 per cent of the Fe nuclei have spins, and the hyperfine coupling for each is of order $2-3 m K)$. However we now assume a second such nanomagnet, at a distance $R$ from the first. The effective bath-mediated interaction $\epsilon(R)$ between them depends strongly on $R$ (as $1 / R^{3}$ ), but even more strongly on the conduction electron density - it goes as $(N(0))^{4}$, where $N(0)$ is the Fermi surface density of states!

Let us suppose that $R=300 \AA$. Then the dipolar interaction between the spins is $V_{d i p} \sim 10^{-5} \mathrm{~K}$. The conduction electron mediated interaction between the 2 nanomagnets in a typical metallic film, with $g \sim 0.1$ (so that $\alpha \sim 20$ ) is then $\epsilon(R) \sim 30 \mathrm{mK}$, ie., 3000 times larger then this (and 30 times larger than $\Delta$ ). In this case it is clear that $\epsilon(R)$ has no real effect on the dynamics of the individual giant spins, which is completely controlled by the very large $\alpha$. As we saw previously, the 2 spins relax independently at high $T$, but at low $T$, their dynamics are frozen into a quantum localised state. As discussed by Prokof'ev and myself [17, this localisation could be of great practical importance for future data storage at the molecular level. Notice however that if $R=100 \AA, \epsilon(R)$ climbs to $1 K$, ie., $\epsilon(R) \sim \Omega_{o}$, and the interaction will completely change the dynamics of the 2 spins - apart from tending to 
unlock the spin dynamics of each, it will also take them out of the Hilbert space of the 2 lowest levels. The dynamics of an array of such spins would be very complicated, even at $T=0$.

Now instead suppose that we drop the conduction electron density by a factor of $\sim 10$, so that $g \rightarrow 0.01$. As previously, this means that $\alpha \rightarrow 0.2$, thereby unlocking the giant spin dynamics at all temperatures. It also means that $\epsilon(R)$ is drastically reduced - it falls by a factor of $10^{4}$ (so that for $R=300 \AA, \epsilon(R)$ is now $3 \times 10^{-6} \mathrm{~K}$ ), and is now smaller than $V_{d i p}$ ! It is also much smaller than $\Delta$, and in fact the dynamics of the spins, in zero applied field, will be controlled at low $T$ by the random nuclear bias, since $E_{o}$ is now the largest energy scale. The spins will again have essentially independent dynamics, when $R=300 \AA$; but now because we are in the perturbative regime.

Notice, however, that if instead $R=100 \AA$, then $V_{\text {dip }}$ rises to $0.3 m K$ (and $\epsilon(R)$ to $0.1 m K$ ), ie., it is of similar size to $\Delta$ and $E_{o}$. Obviously by playing with $\alpha$ and $R$ it is then possible to create a situation where mutually coherent oscillations can occur; otherwise, depending on the exact conditions, we will be either in the correlated relaxation or the locked phases. A nanomagnetic array in the regime might be expected to show very interesting quantum diffusive properties in the $T=0$ limit.

Finally one can imagine further reducing the electron density and/or $S$, to produce a situation where $\alpha \ll 1$, but $V_{d i p}$ is of the same order as $\Delta$ (and both are larger than $E_{o}$ ). This is the "weak coupling" regime. In this case one's first naive guess might be that a nanomagnetic array in this regime should behave coherently, with an energy band of width $\sim \Delta$. It is clear that such an array is not beyond present technology to prepare. This would be a remarkable display of macroscopic coherence behaviour, if true- but have we left anything out of the model?

The answer is of course that we have. We have left out the nuclear dynamics, ie., the dynamics of the background spin bath. This is not so important when $\alpha$ is large, but if $\alpha \ll 1$, it is essential, and it will destroy the coherence of our giant spins, as we shall see in the next subsection (as well as in section 4).

(ii) The Measurement Problem: Let us very quickly look at the analagous implications for our measurement model (which we have largely anticipated already, in introducing it above). Recall that the model discussed in section 2.3 for a quantum measurement involved an overdamped but rapidly responding apparatus (ie., $\Delta_{A}$ large, $\left.\alpha_{A}>1\right)$ and an underdamped system with slow dynamics $\left(\Delta_{s}\right.$ small, $\left.\alpha_{s} \ll 1\right)$. When a measurement is made, an interaction $\mathrm{K}(\mathrm{t})$ is switched on, which is sufficiently strong to allow the apparatus state to correlate with that of the system - however it is not strong enough to cause any transitions in the system state. What actually happens is that as soon as $\mathrm{K}(\mathrm{t})$ exceeds the small $\Delta_{s}$, the system dynamics are frozen, and the apparatus state subsequently evolves to correlate with the frozen system state.

The quantitative calculations of the "Overdamped plus Underdamped" case [57] confirm this picture - I do not propose giving into the details here! There is obvious practical application of these results to the problem of the observation of macroscopic quantum coherent behaviour (on the part of, eg., a SQUID system, or a nanomagnet) the most obvious way of determining the dynamics of such systems is by coupling in another SQUID magnetometer, or a MFM ("Magnetic Force Microscope") to the tunneling system. It is clear that the combined system/apparatus/ environment problem can be described by a PISCES model (provided we can ignore nuclear dynamics - see below)

(iii) Miscellaneous Models: To complete this discussion of results for the dynamics of various commercial oscillator bath models, a brief word on the dynamics of the models noted in section 2.4(c).

The simplest such model is of a "central" harmonic oscillator coupled to a bath of oscillators. If we assume the bilinear coupling $H_{\text {int }}=\sum_{k} c_{k} x_{k} Q$ between the two, the problem is exactly solvable; the details were discussed at great length by Grabert et al. 61. Just as in the standard problem of a classical dissipative oscillator, the results are most easily discussed in the Laplace transform domain. Thus suppose we have a central oscillator of mass $M_{o}$ and frequency $\Omega_{o}$. The classical equation of motion then involves a dissipation coefficient $\eta(\omega)$, in general frequency 
dependent; if $\eta(\omega) \rightarrow \eta$, we have Ohmic dissipation. In terms of the Caldeira and Leggett spectral function $J(\omega)$, the Laplace-transformed function $\eta(s)$ is

$$
\mu(s)=\int_{0}^{\infty} \frac{d \omega}{\pi} \frac{J(\omega)}{\omega} \frac{2 s}{s^{2}+\omega^{2}}
$$

Then the retarded response function $\chi(t)$ of the central oscillator is most conveniently written as $\chi(t)=C(t) \Theta(t)$, where

$$
\begin{aligned}
C(s) & =\int_{0}^{\infty} d t C(t) e^{-s t} \\
& =\frac{1}{M_{o}\left(\Omega_{o}^{2}+s^{2}\right)+s \eta(s)}
\end{aligned}
$$

a result which demonstrates very nicely the correspondence, strongly emphasized by Caldeira and Leggett [13], and mentioned in section 3.2 above, between the classical and quantum behaviour.

In the case of the tunneling problem, there is a huge literature on the dynamics. This work is so extensive that I will simply refer you to the literature here - it is not central to the present discussion, and moreover is discussed in the chapter by Leggett in this book. The original long paper of Caldeira and Leggett 123 is a very good starting point. Hanggi et al. [67] discuss the connection with reaction rate theory, and a very fine set of reviews of the whole field appear in the book edited by Kagan and Leggett [56]. Finally, a good introduction to the theoretical techniques is given by Weiss [64].

For work on the dynamics of a free particle, coupled to an oscillator bath, see the review of Grabert et al. [61] again (as well as the paper of Hakim and Ambegaokar [68]).

So much for the dynamics of systems coupled to oscillator baths; now we turn to the very different effect of the spin bath.

\subsection{Central Spin Model}

In our discussion of oscillator bath models I occasionally warned that the results would be seriously compromised by any spin bath that coupled to the central system. Here we shall see how this happens, and review results for the dynamics of a central system coupled to a spin bath. It is crucial here to recall the essential difference between the oscillator and spin bath models- whereas the oscillators are only very weakly affected by the central system (so that their spectra and dynamics are hardly altered), the spins in the spin bath have their spectra and dynamics changed completely.

The discussion of the present section is based on papers by myself and Prokof'ev [14, 16, 17, 18] (see also ref. [69]), for which see the gory details - here I attempt an intuitive perspective on these, and briefly note their application to nanomagnets (discussed in detail in Chapter 4), and SQUIDs.

\section{4(a) THREE LIMITING CASES:}

Let us first recall the low-energy effective Hamiltonian we derived for the central spin model, whose derivation was described in section 2.2. The Hamiltonian, in the absence of an external field, was

$$
H_{\mathrm{eff}}=\left\{2 \Delta_{o} \hat{\tau}_{-} \cos \left[\pi S-i \sum_{k}\left(\alpha_{k} \vec{n} \cdot \hat{\vec{\sigma}}_{k}+i \beta_{o} \vec{n} \cdot \vec{H}_{o}\right)\right]+H . c .\right\}
$$




$$
+\frac{\hat{\tau}_{z}}{2} \sum_{k=1}^{N} \omega_{k}^{\|} \vec{l}_{k} \cdot \hat{\vec{\sigma}}_{k}+\frac{1}{2} \sum_{k=1}^{N} \omega_{k}^{\perp} \vec{m}_{k} \cdot \hat{\vec{\sigma}}_{k}+\sum_{k=1}^{N} \sum_{k^{\prime}=1}^{N} V_{k k^{\prime}}^{\alpha \beta} \hat{\sigma}_{k}^{\alpha} \hat{\sigma}_{k^{\prime}}^{\beta} .
$$

where $\overrightarrow{\hat{\tau}}$ represents the central spin, and the $\left\{\sigma_{k}\right\}$ the spin bath variables; the notation is that of section 2.2 .

What we wish to do here is calculate the dynamics of $\vec{\tau}$ after integrating out the bath variables - our problem is similar to that addressed in section 3.2, only the bath has changed. I shall describe the physics in a tutorial manner, by first introducing you to 3 limiting cases, and then showing how the complete solution is an amalgam of these cases.

(i) Topological Decoherence Limit: This limiting case removes all of the static coupling between the central spin and the spin bath, and also removes the intrinsic spin bath dynamics, by supressing $V_{k k^{\prime}}^{\alpha \beta}$. The resulting Hamiltonian is

$$
H_{\mathrm{eff}}^{\text {top }}=2 \Delta_{o} \hat{\tau}_{x} \cos \left[\pi S-i \sum_{k=1}^{N} \alpha_{k} \vec{n}_{k} \cdot \hat{\vec{\sigma}}_{k}\right],
$$

The essential feature of this model is that all of the spin bath dynamics is driven by the central spin - moreover, from the form of $H_{e f f}$, we see that to the Berry phase $\pi S$ of the central spin is added a complex phase $-i \Sigma_{k} \alpha_{k} \vec{n}_{k} \cdot \overrightarrow{\sigma_{k}}$ coming from the bath. Thus the main effect of the bath transitions is to add a random phase to the action incurred during each central spin transition - in effect the topological phase in the system dynamics is randomised. Both the physical and mathematical aspects of this case have been discussed in some detail in the original references, and I will not repeat this here. The main ideas can be entirely understood in the special case where we choose $\alpha_{k}$ to be entirely imaginary; and I will simply now refer to this imaginary quantity as $\alpha_{k}$. The solution can then be written formally as

$$
P_{11}(t)=\frac{1}{2}\left\{1+\left\langle\cos \left[4 \Delta_{o} t \cos \left(\Phi+\sum_{k=1}^{N} \alpha_{k} \vec{n}_{k} \cdot \hat{\vec{\sigma}}_{k}\right)\right]\right\rangle\right\},
$$

where $\langle\ldots\rangle$ is an average over the environmental states, and we have made the replacement $\pi S \rightarrow \Phi$, to take account of any high-frequency $\left(>\Omega_{o}\right)$ renormalization of the central spin Haldane phase, caused by the nuclear bath. In the present model all bath states are degenerate, and this average is trivial - it can be written as a weighted integration over topological phase $\varphi$, as

$$
\begin{aligned}
P_{11}(t) & =\sum_{m=-\infty}^{\infty} F_{\lambda^{\prime}}(m) \int_{0}^{2 \pi} \frac{d \varphi}{2 \pi} e^{i 2 m(\Phi-\varphi)}\left\{\frac{1}{2}+\frac{1}{2} \cos \left(2 \Delta_{o}(\varphi) t\right)\right\} \\
& =\frac{1}{2}\left\{1+\sum_{m=-\infty}^{\infty}(-1)^{m} F_{\lambda^{\prime}}(m) e^{i 2 m \Phi} J_{2 m}\left(4 \Delta_{o} t\right)\right\},
\end{aligned}
$$

where we define

$$
\lambda=\frac{1}{2} \sum_{k}\left|\alpha_{k}\right|^{2} ; \quad F_{\lambda}(\nu)=e^{-4 \lambda \nu^{2}}
$$

As mentioned in section $2.2, \lambda$ is the mean number of bath spins flipped each time $\vec{S}$ flips. If $\lambda>1$, but not too large; I will call this the intermediate coupling limit. Then

$$
F_{\lambda}(\nu)=\delta_{\nu, 0}+\text { small corrections } \quad \text { (intermediate) } .
$$


so that, very surprisingly, we get a universal form in the intermediate coupling regime for $P_{11}(t)$ (here $\eta(x)$ is the step function):

$$
P_{11}(t) \longrightarrow \frac{1}{2}\left[1+J_{0}\left(4 \Delta_{o} t\right)\right] \equiv \int \frac{d \varphi}{2 \pi} P_{11}^{(0)}(t, \Phi=\varphi)
$$

(compare the angular average of the coherent series in (94)), ¿From this we can also compute the absorption of the 2-level giant spin, as a function of frequency, by Fourier transforming; we get

$$
\chi^{\prime \prime}(\omega) \longrightarrow \frac{2}{\left(16 \Delta_{o}^{2}-\omega^{2}\right)^{1 / 2}} \eta\left(4 \Delta_{o}-\omega\right) \quad \text { (intermediate) } .
$$

We plot this universal form in Fig.15.

FIG. 15: The universal form for the power absorption $\chi^{\prime \prime}(\omega)$ in the case of pure topological decoherence.

The physics of this universal form is simply one of phase cancellation., As explained in refs. 14, 15., this phase cancellation arises because successive flips of $\vec{S}$ cause, in general, a different topological phase to be accumulated by the spin environment, so that when we sum over successive instantons for $\vec{S}$, we get phase randomisation and hence loss of coherence. The only paths that can then contribute to coherent oscillations in $P_{11}(t)$ are those for which the number of clockwise and anticlockwise flips in the spin bath are equal - in this case the topological phase "eaten up" by the environmental spins is zero. By looking at expression (97) one sees another way of describing this- the universal behaviour comes from complete phase randomisation 15$]$, so that all possible phases contribute equally to the answer! The final form shows decaying oscillations, with an envelope $\sim t^{-1 / 2}$ at long times. This decay can also be understood [16] by noting that the "zero phase" trajectories that contribute to $P_{11}$ constitute a fraction $(2 s) ! /\left(2^{s} s !\right)^{2} \sim s^{-1 / 2}$ of the total number of possible trajectories, where $s \sim \Delta_{o} t$. Because of this decay, the $\delta$-function peak in the spectral function at $\omega=4 \Delta_{o} \cos \Phi$ is now transformed to the spectral function of a 1-dimensional tight-binding model. 
One should also note that in the the strong coupling limit, when $\left|\alpha_{k}\right| \rightarrow \pi / 2$, so that each spin rotates adiabatically with $\vec{S}$, we simply have

$$
P_{11}(t)=\frac{1}{2}\left[1+\cos \left(4 \Delta_{o} t \cos \tilde{\Phi}\right)\right],
$$

where $\tilde{\Phi}=\pi S+N \pi / 2$, i.e., the Haldane/Kramers phase is now $\tilde{\Phi}$, with the extra phase coming from the $N$ bath spins which rotate rigidly with $\vec{S}$.

Recall that the above results are given for the special case where $\alpha_{k}$ is pure imaginary; for the general complex case see the original work.

(ii) Orthogonality Blocking Limit: Let us now consider the case where again all bath dynamics is suppressed $\left(V_{k k^{\prime}}^{\alpha \beta}=0\right)$, but now we retain only the static diagonal part of $H_{\text {eff }}$, ie., we assume

$$
\begin{gathered}
H_{\text {eff }}=2 \Delta_{\Phi} \tau_{x}+\hat{\tau}_{z} \frac{\omega_{o}^{\|}}{2} \sum_{k=1}^{N} \vec{l}_{k} \cdot \hat{\vec{\sigma}}_{k}+\frac{\omega_{o}^{\perp}}{2} \sum_{k=1}^{N} \vec{m}_{k} \cdot \hat{\vec{\sigma}}_{k} . \\
\Delta_{\Phi}=\Delta_{o} \cos \Phi,
\end{gathered}
$$

where $\omega_{o}^{\|} \gg \omega_{o}^{\perp}$; in $(100)$ we use a "Kramers renormalised" matrix element $\Delta_{\Phi}$. The significance of the finite but small transverse term $\omega_{o}^{\perp}$, is that in any case where the initial and final fields $\vec{\gamma}_{k}$ acting on $\vec{\sigma}_{k}$ are not exactly parallel or antiparallel, the diagonal coupling must have a transverse part (cf. section 2.2(b)).

Now in this case no transitions occur in the spin bath when the central spin $\vec{S}$ flips. However the bath spins still play a crucial role, because the transverse "field" term $\omega_{o}^{\perp} \sum_{k} \hat{\sigma}_{k}^{x}$ changes the motion of $\vec{S}$. There is perhaps a temptation to treat this as nothing but the simple problem of the changed motion of $\vec{S}$ in this transverse field. However this is quite wrong - if we do this we forget that the transverse term is coming from a set of spin variables (the $\left\{\overrightarrow{\hat{\sigma}}_{k}\right\}$ ) to each of which is associated a wave-function $\mid \Phi_{k}>$. The crucial point here is that in the presence of the transverse coupling, there is a mismatch between the initial state $\left|\Phi_{k}^{i n}\right\rangle$ (before $\vec{S}$ flips) and the final state $\mid \Phi_{k}^{f i n}>$ (after it flips); in general $\left|\left\langle\Phi_{k}^{i n} \mid \Phi_{k}^{f i n}\right\rangle\right|<1$ if $\omega_{o}^{\perp} \neq 0$. When we average over all bath spins, this seriously alters the system dynamics. Since $\omega_{o}^{\perp} \ll \omega_{o}^{\|}$, we define an angle $\beta_{k}$, describing this mismatch between the initial and final states of $\vec{\sigma}_{k}$, according to

$$
\cos 2 \beta_{k}=-\hat{\vec{\gamma}}_{k}^{(1)} \cdot \hat{\vec{\gamma}}_{k}^{(2)}
$$

where $\hat{\vec{\gamma}}_{k}^{(1)}$ and $\hat{\vec{\gamma}}_{k}^{(2)}$ are unit vectors in the direction of the initial and final state fields acting on $\vec{\sigma}_{k}$ (and here we assume these are almost exactly antiparallel). If we assume that $\beta_{k} \ll 1$, then the total mismatch, of the usual "Debye-Waller" or "Franck-Condon" form, is just $e^{-\kappa}$, where

$$
\kappa=\frac{1}{2} \sum_{k} \beta_{k}^{2}
$$

More generally, if the mismatch angles $\beta_{k}$ are not so small, we just have

$$
e^{-\kappa}=\prod_{k=1}^{N} \cos \beta_{k} .
$$


Because of the similarity to the famous "orthogonality catastrophe" considered by Anderson (in which the electronic phase shift $\delta_{k}$ is analogous to the $\beta_{k}$ considered here), we have called this limit that of "orthogonality blocking". However the physics here is rather different because the bath spins behave quite differently from electrons (which, we recall, map to an oscillator bath). As shown in the original work, [] the easiest way to understand and to solve for the dynamics of $\vec{S}$ is to notice that $\mid \Phi_{k}^{\text {fin }}>$ is produced from $\mid \Phi_{k}^{i n}>$ by the unitary transformation:

$$
\left|\vec{\sigma}_{k}^{f i n}\right\rangle=\hat{U}_{k}\left|\vec{\sigma}_{k}^{i n}\right\rangle=e^{-i \beta_{k} \hat{\sigma}_{k}^{x}}\left|\vec{\sigma}_{k}^{i n}\right\rangle
$$

Thus, mathematically, the problem is identical to one in which there is an amplitude $\beta_{k}$ for $\vec{\sigma}_{k}$ to flip each time $\vec{S}$ flips, and where typically $\beta_{k} \ll 1$. Physically, the mismatch between the 2 states arises because the fields $\vec{\gamma}_{k}^{i n}$ and $\vec{\gamma}_{k}^{\text {fin }}$ acting on $\vec{\sigma}_{k}$ are neither parallel or exactly antiparallel. Thus if $\sigma_{k}$ is initally aligned with $\vec{\gamma}_{k}^{i n}$, it finds itself slightly misaligned with $\vec{\gamma}_{k}^{f i n}$ after $\vec{S}$ flips (semiclassically, $\vec{\sigma}_{k}$ must start precessing in the field $\vec{\gamma}_{k}^{f i n}$ ).

This problem is thus similar to that of topological decoherence (with $\beta_{k}$ replacing $\alpha_{k}$, and $\kappa$ replacing $\lambda$ ), except that we now have the added complication that the spin bath states are not at all degenerate - in fact they are split by the large longitudinal coupling $\omega_{o}$. Recall (end of section 2.2) that this spreads the nuclear bath states over a large energy range $E_{o} \sim \omega_{o} N^{\frac{1}{2}}$, so that in zero applied field, $S$ cannot make any transitions at all unless the internal spin bath field $\epsilon=\omega_{o} \sum_{k} \sigma_{k}^{z} \tau_{k}^{z}$ bias energy is the same before and after $\vec{S}$ flips. Otherwise, since typically $\omega_{o} \gg \Delta$, the difference $2 \epsilon$ between the intial and final energies of $\vec{S}$ simply blocks all tunneling. This we immediately see that we require that either (i) the net polarisation $\tilde{M}=\sum_{k} \sigma_{k}^{z}$ of the spin bath is zero both before and after $\vec{S}$ flips (so $\epsilon=0$ ); or (ii) $\tilde{M}$ changes from $M$ to $-M$ when $\vec{S}$ flips - meaning that at least $M$ spins flip (and $\epsilon=M \omega_{o}$ before and after $\vec{S}$ flips).

For details of the resulting calculations of $P_{\Uparrow \Uparrow}(t)$, and the resulting rather bizarre behaviour, I refer to the original papers [16, 18].

(iii) Degeneracy Blocking Limit: Finally let us consider the case where again $V_{k k^{\prime}}^{\alpha \beta}=0$ (no spin bath dynamics), and both the non-diagonal terms and the transverse diagonal term $\omega_{k}^{\perp}$ are zero. Thus we have

$$
H_{\mathrm{eff}}=2 \Delta \tau_{x}+\frac{1}{2} \tau_{z} \sum_{k=1}^{N} \omega_{k}^{\|} \hat{\sigma}_{k}^{z}
$$

with a spread of values of $\omega_{k}^{\|}$of

$$
\sqrt{\sum_{k}\left(\omega_{k}^{\|}-\omega_{o}\right)^{2}} \equiv N^{1 / 2} \delta \omega_{o}
$$

i.e., a distribution of width $\delta \omega_{o}$.

Clearly, this Hamiltonian is identical to the standard biased two-level system, with the bias energy $\epsilon$ depending on the particular environmental state; thus $\epsilon=\sum_{k=1}^{N} \omega_{k}^{\|} \sigma_{k}^{z}$. The introduction of this spread is to destroy the exact degeneracy between states in the same polarisation group. For coherence to take place, we require the initial and final states to be within roughly $\Delta$ of each other.

The crucial point is of course that $\omega_{o}^{\|} \gg \Delta$ in most cases, and in fact one often has $\delta \omega_{o}^{\|}>\Delta$ (thus, in a nanomagnet, $\delta \omega_{o}^{\|}$comes not only from the variation of the local contact hyperfine couplings $\omega_{j} \vec{s}_{j} \cdot \vec{I}_{j}$, but also from the large variety of weaker transfer hyperfine couplings between the $\vec{s}_{j}$ and nuclei on other non-magnetic sites). A 
suitable dimensionless parameter which describes the extent of the spread in the $\omega_{k}^{\|}$, around the "central" value $\omega_{o}$, is $\mu=N^{\frac{1}{2}} \delta \omega_{o} / \omega_{o}$. In the limit where $\mu \rightarrow 0$, and then the $2^{N}$ nuclear levels are organised into a set of sharp lines, each one corresponding to a particular polarisation group- however this case is rather academic. More generally we define a density of states function $W(\epsilon)$ for the nuclear levels in the presence of the hyperfine coupling (Fig.16).

FIG. 16: The density of states function $W(\epsilon)$ for the nuclear multiplet for 2 different values of the parameter $\mu$ (see text). The case $\mu<1$ is somewhat academic, and so $W(\epsilon)$ is Gaussian in practise.

If $\mu<1$ the lines still can be seen, with however the different polarization groups overlapping. If $\mu>1$, however (and this is almost invariably the case in any real system), this structure disappears and we are left with a Gaussian form [14, 16]:

$$
\begin{gathered}
W(\epsilon)=\left(\frac{2}{\pi E_{o}^{2}}\right)^{1 / 2} \exp \left(-2 \epsilon^{2} / E_{o}^{2}\right) \\
E_{o}=\omega_{o}^{2} N
\end{gathered}
$$

This form was already mentioned in section 2.2. In nanomagnetic molecules $\mu$ is usually considerably greater than unity, even for small magnetic molecules, because of the large number of protons in the molecule and its ligands. In nanomagnetic grains the nuclei of $O$ ions (as well as protons in hydrated systems) as well as other magnetic species, have the same effect.

The dynamics of an ensemble of nanomagnets, each described by the above Hamiltonian, but averaged over the nuclear distribution $W(\epsilon)$ in a thermal ensemble, is then quite trivial to obtain - it is just the weighted ensemble average over the correlation function of a simple 2-level system in this internal bias field $\epsilon$. In the usual case where $k T \gg \Delta$, we then have

$$
P_{11}(t)=\int d \epsilon W(\epsilon) \frac{e^{-\beta \epsilon}}{Z(\beta)}\left[1-\frac{2 \Delta_{\Phi}^{2}}{\epsilon^{2}+4 \Delta_{\Phi}^{2}}\left(1-\cos \left(2 t \sqrt{\epsilon^{2}+4 \Delta_{\Phi}^{2}}\right)\right)\right]
$$




$$
\longrightarrow \quad 1-2 A \sum_{k=0}^{\infty} J_{2 k+1}\left(4 \Delta_{\Phi} t\right) .
$$

where the reduction factor $A$ is just

$$
A=2 \pi \Delta_{\Phi} /\left(\omega_{o} \sqrt{2 \pi N}\right) \ll 1 .
$$

and the spectral function is given by another universal form, viz:

$$
\chi^{\prime \prime}(\omega)=A \frac{8 \Delta_{\Phi}}{\omega \sqrt{\omega^{2}-16 \Delta_{o}^{2}}} \eta\left(\omega-4 \Delta_{\Phi}\right),
$$

FIG. 17: The universal form for $\chi^{\prime \prime}(\omega)$ in the case of pure degeneracy blocking. Notice that absorption only occurs above the threshold defined by $\Delta_{o}$.

The result here is very easy to understand. Only those nanomagnets near resonance (ie., with $\epsilon \sim \Delta$ or less) stand much of a chance of tunneling, since tunneling requires near degeneracy between intial and final states. Those systems which do not have this degeneracy are "degeneracy blocked"; and since $\Delta \ll E_{o}$, almost all of them are frozen. This result of course arises because the only dynamics in this limiting model comes from the tunneling term itself. As soon as we give the spin bath some dynamics (either by reintroducing the non-diagonal terms, or even more importantly, by making $V_{k k^{\prime}}^{\alpha \beta}$ non-zero, so that the nuclear bath has independent dynamics), the blocking will be relieved, as we shall now see.

3.4(b) GENERAL SOLUTION The discussion of the 3 limiting cases given above allows us to give a simple intuitive picture of the general solution to the problem (readers wanting details, proofs, etc., should go to the original papers $[17,18,69]$ ). 
(i) Independent Bath Spins: Suppose we start with the (often rather academic) case where the bath spins do not interact at all with each other (formally, this means $V_{k k^{\prime}}^{\alpha \beta}$ is much less than all other energy scales, in particular $\Delta$ ). The results are so pretty, and intuitively revealing, that I will quickly describe them to you.

Each of the 3 limiting cases above corresponds to one physical mechanism (already previewed back in our initial discusion of this model, in section 2.2(b)). Now the magic is that the general solution is simply obtained by combining them! Mathematically, each mechanism corresponds to a particular statistical average:

$$
\begin{aligned}
& \text { (a) A "topological phase average" given by } \sum_{\nu=-\infty}^{\infty} F_{\lambda^{\prime}}(\nu) \int \frac{d \varphi}{2 \pi} e^{i 2 \nu(\Phi-\varphi)} \\
& \text { (b) An " orthogonality average" given by } 2 \int_{0}^{\infty} d x x e^{-x^{2}} \\
& \text { (c) A "bias average" } \int d \epsilon W(\epsilon) \frac{e^{-\beta \epsilon}}{Z(\beta)}
\end{aligned}
$$

Apart from the orthogonality average (the derivation of which is too complicated to explain here), you have just seen these above. Now, consider a simple 2-level system in a bias $\epsilon$, but with a renormalised tunneling splitting

$$
\Delta_{M}(\varphi, x)=2 \tilde{\Delta}_{o}\left|\cos (\varphi) J_{M}(2 x \sqrt{\gamma})\right|,
$$

and associated energy splitting $E_{M}^{2}(\varphi, x)=\Delta_{M}^{2}(\varphi, x)+\epsilon^{2}$. This tunneling matrix element now depends explicitly on the topological phase, on the nuclear polarisation $M$, and on the orthogonality variable $x$. The parameter $\gamma$ is just

$$
\gamma= \begin{cases}\lambda & \text { if } \lambda \gg \kappa \text { (topological decoherence regime) } \\ \kappa & \text { if } \kappa \gg \lambda \text { (orthogonality blocking regime) }\end{cases}
$$

Recall now the very simple dynamics of an isolated biased 2-level system (eqtn. (28)); the probability that the system stays in state $|\uparrow\rangle$ after a time $t$ is $P_{11}^{(0)}(t)=1-(\Delta / E)^{2} \sin ^{2}(E t)$. Then, perhaps amazingly, the same function for the central system, after all averaging over the bath spins is carried out, is nothing but a generalisation of this; one gets

$$
P_{11}(t ; T)=1-\int d \epsilon W(\epsilon) \frac{e^{-\beta \epsilon}}{Z(\beta)} \sum_{M=-N}^{N}\left(1-P_{M}\left(t, \epsilon-M \omega_{o}\right)\right) ;
$$

in which the probability relaxation function for a given polarisation group $M$ is

$$
P_{M}(t ; \epsilon)=2 \int_{0}^{\infty} d x x e^{-x^{2}} \sum_{\nu=-\infty}^{\infty} F_{\lambda^{\prime}}(\nu) \int \frac{d \varphi}{2 \pi} e^{i 2 \nu(\Phi-\varphi)}\left[1-\frac{\Delta_{M}^{2}(\varphi, x)}{E_{M}^{2}(\varphi, x)} \sin ^{2}\left(E_{M}(\varphi, x) t\right)\right],
$$

That is all! Perhaps even more amazing, these integrals and sums can be done analytically for almost all of the parameter regime (which, amusingly, is more than one can say for the spin-boson model, which just goes to show that a simple Hamiltonian does not always have simple dynamics!). The physical interpretation of this result is of course obvious, just by looking at our 3 limiting cases again; I will not go over it again. If you want to see analytic formulae and pictures, see Prokof'ev and Stamp [17, 18, 69; you will find that in some cases they do not look anything like what you can get from a spin-boson model! 
This of course raises the question- can this "central spin" model ever behave like a spin-boson model? The answer is yes. Lets first think about this physically- we wish the spins to somehow behave like a set of oscillators, weakly coupled to the central spin. If you recall the form of the Caldeira-Leggett spectral function $J(\omega)$, involving a coupling constant squared, divided by an energy denominator (cf. 2nd-order perturbation theory), then it is clear how this can happen, for in our case the 2 relevant parameters are $\omega_{k}^{\|}$and $\omega_{k}^{\|}$, and each is capable of playing the role of the coupling $c_{k}$ (with the other playing the role of the energy denominator). However this only works if the coupling is small (compared to $\Delta$ ). An example is provided by the SQUID model we considered in section 2.3(a), summarized in the effective Hamiltonian (41); recall that there $\omega_{k}^{\|} / \omega_{k}^{\perp} \ll 1$, and both are very small. This problem (which is related to that studied by Caldeira et al. 20]), is easily solved by writing $P_{11}(t)$ as

$$
\begin{array}{r}
P_{11}(t)=\sum_{n m}^{\infty}\left(i \Delta_{o}\right)^{2(n+m)} \int_{0}^{t} d t_{1} \ldots \int_{t_{2 n-1}}^{t} d t_{2 n} \int_{0}^{t} d t_{1}^{\prime} \ldots \int_{t_{2 m-1}^{\prime}}^{t} d t_{2 m}^{\prime} \mathcal{F}\left(Q, Q^{\prime}\right) \\
\mathcal{F}\left(Q, Q^{\prime}\right)=\prod_{k}\left\langle\hat{U}_{k}\left(Q_{(n)}, t\right) \hat{U}_{k}^{\dagger}\left(Q_{(m)}^{\prime}, t\right)\right\rangle,
\end{array}
$$

where as before $Q_{(n)}(t)$ refers to a path of the central system containing $n$ instanton jumps, but now $\hat{U}_{k}\left(Q_{(n)}, t\right)$ refers to the time evolution of the $k$-th environmental spin (not oscillator!), under the influence of the central spin (cf eqtn. (77)). In the present case this is

$$
\hat{U}_{k}\left(Q_{(n)}, t\right)=T_{\tau} \exp \left\{-i \int_{0}^{t} \frac{d s}{2}\left[\omega_{k}^{\perp} \hat{\sigma}_{k}^{z}+Q_{(n)}(s) \omega_{k}^{\|} \hat{\sigma}_{k}^{x}\right]\right\}
$$

In general this formula for the "influence functional" is intractable (hence our alternative approach above!). However in the present case, expanding to 2 nd order in $\omega_{k}^{\|}$, one gets at $T \gg \omega_{k}^{\perp}$ that

$$
\mathcal{F}\left(Q, Q^{\prime}\right)=\exp \left\{-\sum_{k} \frac{\left(\omega_{k}^{\|}\right)^{2}}{8}\left|\int_{0}^{t} d s e^{2 i \omega_{k}^{\perp} s}\left[Q_{(n)}(s)-Q_{(m)}^{\prime}(s)\right]\right|^{2}\right\},
$$

Now in general this is still hard to solve; but for the SQUID it is simple, because even though $\omega_{k}^{\|}$is extremely small, there are so many nuclear spins that the parameter $\gamma$ defined by $\gamma^{2}=\left[\left(\sum_{k} \omega_{k}^{\|}\right)^{2} / 2\right]^{1 / 2}$ is not (for our previous model parameters $\gamma \sim 5 \times 10^{-4} \mathrm{~K}$, similar to the total longitudinal bias produced by the nuclei), and so we expect $\gamma \gg \omega_{k}^{\perp}, \Delta_{o}$ (unless $\Delta_{o}$ is very large indeed). In this case it is easy to see that $\mathcal{F}\left(Q, Q^{\prime}\right) \sim e^{-\gamma^{2}\left(t-t^{\prime}\right)^{2}}$, ie., the influence functional decays (and dephases everything) before anything else can move (which, incidentally, is why we can ignore the nuclear bath dynamics in studying this case). In path integral language, kink-antikink pairs are closely bound (justifying a "NIBA"); more prosaically, we get a fast "motional narrowing" relaxation, with $P_{11}(t)=1 / 2\left(1-e^{-t / \tau_{R}}\right)$, and with the relaxation time given just by the integral

$$
\tau_{R}^{-1}=2 \Delta_{o} \int_{0}^{\infty} e^{-\Gamma^{2}\left(t_{2}-t_{1}\right)^{2}} \equiv \frac{\sqrt{\pi} \Delta_{o}^{2}}{\Gamma}
$$

This result does not give out much hope for the experimental search for MQC in SQUIDs; it indicates coherence will be destroyed by nuclear spins in a time scale $\ll \Delta_{o}^{-1}$.

\section{(ii) Influence of Spin Bath Dynamics:}


Suppose we now go back to the full spin bath problem. What will be the influence of the small dynamical term $V_{k k^{\prime}}^{\alpha \beta}$ on the results. The answer is that it can be profound, because in a situation where the central system is more or less blocked from transitions by the strong degeneracy blocking field, the weak residual bath dynamics can "scan" the bias energy until the system finds a resonance. In general this can happen with the simultaneous flipping of some bath spins, so that the resonance condition must include the change in energy of the nuclear bath as well; ie., we will end up summing over polarisation groups $M$. Thus, we have an external bias $\xi$, an internal longitudinal bias $\epsilon(t)=\epsilon+\delta \epsilon(t)$ (where $\delta \epsilon(t)$ represents the time dependent fluctuations coming from the internal spin bath dynamicsor, at higher $T$, from external couplings which drive the spin bath, ie., $T_{1}$ - processes in the case of a nuclear bath), and the condition for resonance is that $\xi+\epsilon+M \omega_{o}+\delta \epsilon(t) \sim 0$. Moreover, the system should stay in resonance long enough to give the central spin a chance to tunnel!

This point is of very great practical importance; in fact it is obviously going to be entirely responsible for the existence of any dynamics in the low- $T$ limit (as was discussed in some detail by Prokof'ev and myself, a while ago, in the context of nanomagnets [17), since every other relaxation mechanism is frozen! What is found is that for a single isolated nanosystem (ie., a nanomagnet), in the Quantum regime, the new correlation function should be given by the simple incoherent relaxation form

$$
P_{11}(t)=\sum_{M} w(T, M) \int x d x e^{-x^{2}} \sum_{\nu=-\infty}^{\infty} \int \frac{\varphi}{2 \pi} F_{\lambda^{\prime}}(\nu) e^{i 2 \nu(\Phi-\varphi)}\left[1+e^{-t / \tau_{M}(x, \varphi)}\right],
$$

where the relaxation time $\tau_{M}(x, \varphi)$ for the $M$-th polarisation group is

$$
\tau_{M}^{-1}(\xi, \varphi)=2 \Delta_{M}^{2}(x, \varphi) / \pi^{1 / 2} \xi_{o}
$$

The derivation is simple but too long to be repeated here. The parameter $\xi_{o} \sim N^{1 / 2} \delta \omega_{o}$ is a measure of the total energy spread of each polarisation group in the density of states (recall Fig.16 ). In the absence of any such spread, we have $\xi_{o} \sim N^{1 / 2} T_{2}^{-1}$, where the residual couplings between the bath spins give the total bath spectrum a linewidth $\xi_{o}$; here $T_{2}^{-1} \sim V_{k k^{\prime}}^{\alpha \beta}$. We use this notation because in the case of a nuclear spin bath, this linewidth is parametrised (and in principle defined experimentally) by the transverse nuclear spin diffusion time $T_{2}$. Typically $T_{2}^{-1} \sim 10-100 k H z$ in this case, although it can vary a lot. Notice that these bath fluctuations are in the high- $T$ limit for anyone except a nanoKelvin experimentalist- we cannot make them go away! This result is easiest to understand in the limit where both degeneracy blocking and topological decoherence are absent (no nuclei coflip with $\vec{S}$, and $H_{o}(\vec{S})$ has axial symmetry). Then the averages collapse, and we get a unique relaxation rate given by $\tau_{o}=2 \Delta_{o}^{2} / \pi^{1 / 2} \xi_{o}$. This is of course just the overlap between initial and final states in a typical bias $\xi_{o}$, but be careful- it is a relaxation rate, involving the irreversible passage of the sytem from one state to the other (with an energy absorption $\sim \xi_{o}$ by the spin bath). The difference with a simple overlap integral becomes clear if the calculation is redone in a finite bias $\xi$ (see Prokof'ev and Stamp [17]); then we get instead that

$$
\tau(\xi)=\tau_{0}^{-1} e^{-|\xi| / \xi_{o}}
$$

and we see that the rate falls of exponentially with the bias, as we take the system away from any possibility of reaching the resonant tunneling window, during its peregrinations over an energy range $\xi_{o}$. This formula is essential to understanding the low- $T$ dynamics of any system described by a central spin model.

This concludes the very long discussion of canonical models and their dynamics. Enough, then, of Olympian theory- let's now get down to a merely Homeric epic, with some real physics (including experiments)! 


\section{Quantum Nanomagnetism}

Before plunging into a new field, it is certainly worth asking what fundamentally new ideas may come out of it. So why study quantum nanomagnets? Here are a number of reasons, each of which will appeal to different tastes:

(i) They constitute a (very rich) testing ground for theorists interested in tunneling in complex systems; one can attack on many different fronts (numerical, analytical, experimental), and there is an almost inexhaustible variety of real examples to work with. The spin Hamiltonians for magnetic nanomolecules lend themselves well to studies in quantum chaos.

(ii) Much more than superconductors, they allow detailed study of the crossover between quantum and classical properties of physical systems, as one changes the system size, temperature, and coupling constants. Understanding this crossover is not only important for the resolution of the notorious quantum measurement problem (and a better understanding of how decoherence works at this scale); it will also have enormous practical consequences in the next few decades, with the coming of quantum nanomagnetic devices. Unravelling the relevant physics promises to be one of the most exciting challenges in condensed matter physics.

(iii) In the field of magnetism itself, this crossover is of central interest; it has been highlighted by the discovery of high-temperature superconductors, heavy fermions, 1-dimensional magnets (spin chains), and a variety of "frustrated magnets", many of which show tendencies towards "spin liquid" behaviour (ie., quantum-disordered magnetic states), even at the macroscopic level. More recently there has been the discovery, (in Mn-perovskite layered magnets, having the same structure as the high-Tc La-perovskite superconductors), of "collosal magnetoresistance" phenomena; and the preparation of "spin ladder" materials, which are essentially sets of coupled spin chains. There is no question that the study of these quantum magnetic materials (and their remarkable superconducting properties when doped), is the central focus of most of the condensed matter research around the world at present. It is quite extraordinary that almost all of these materials (including the magnetic molecules to be discussed presently) are Transition Metal Oxides, at or near a metal-insulator transition, with magnetism controlled by superexchange, and great sensitivity to doping. Why all his should be, is one of the great mysteries in physics at the end of the 20th century.

(iv) Some magnetic systems offer the prospect of observing genuinely macroscopic quantum phenomena, in the sense discussed by Leggett [41]. The most likely possibility here is in the tunneling of domain walls (or possibly their quantum nucleation), which at least theoretically should occur on a large scale [42], particularly in systems isotopically purified of nuclear spins [28]. Some promising experiments in this direction have been done already [10, 11].

¿From this short list we see that nanomagnets appeal to physicists (and even philosophers of science) interested in the mysteries of quantum mechanics and the measurement problem; and at the same time to industrialists and venture capitalists, investing in the technology of the next century. This curious mixture is not infrequent in subjects at the frontier of science.

In the following I will give an overview of 2 important themes in this relatively new field, aimed at the non-specialist. These show (sometimes rather dramatically) how the concepts discussed in Chapters 2 and 3 can be applied to real physical systems. Incidentally, although some of the experiments are understood theoretically, others are not, and there are some intriguing outstanding mysteries. I will start with the now quite extensive results (theoretical and experimental) on quantum relaxation in magnetic micromolecules and grains; I will argue that these are fairly well understood. Then we look at "macroscopic coherence" experiments in ferritin macromolecules, where the gap between theory and experiment is still controversial. I do not discuss other areas (eg., domain wall tunneling), simply because of a lack of space. 


\subsection{Resonant Tunneling Relaxation}

Let us start with a set of experiments in which no coherence exists at all. Considerable press (some of it highly misleading) has been devoted recently to a series of experiments [1]-7 7 which have shown, with steadily increasing clarity, the role of tunneling in the relaxation of magnetisation in an initially polarized sample of magnetic molecules. These experiments did not appear in a vacuum, but rather in the context of previous experimental efforts to see tunneling in magnetic particles and in the biological macromolecule ferritin (see section 4.3 below). The possibility of doing tunneling relaxation experiments on molecule crystals of large-spin nanomolecules relies essentially on recent advances in the preparation of such molecules by molecular chemists [].

There have also been a large number of tunneling relaxation experiments on nanomagnetic particles. I shall make no attempt to review all of these; however some of them are very interesting, specially as they indicate what remarkable possibilities exist for the future.

\section{1(a) MAGNETIC NANOMOLECULES- STRUCTURE and EXPERIMENTS}

I will only touch the surface here of a huge field, which was for many years the purview of chemists and the occasional molecular physicist. Reviews of some of the chemistry are given by, eg., Gatteschi et al [70]; and I would particularly recommend the book of Kahn [71]. The low- $T$ experiments are too new to have been reviewed.

(i) Spin Structure at Low Energies: The first experiments were done on the "Mn-12" system, first in powder samples and then in large single crystals (refs. [1]-[6]). Early theoretical studies of this molecule, by the Grenoble-Firenze group, [] led to the proposal of a "giant spin" Hamiltonians $H_{o}(\vec{S})=\left({ }^{\|} H_{o}^{(2)}+{ }^{\perp} H_{o}^{(4)}\right)$, where

$$
\begin{gathered}
{ }^{\|} H_{o}^{(2)}=-(D / S) S_{z}^{2} \\
{ }^{\perp} H_{o}^{(4)}=-\left(K_{4} / S^{3}\right)\left[S_{+}^{4}+S_{-}^{4}\right]
\end{gathered}
$$

and in which $\mathrm{S}=10, \mathrm{D} \sim 6-7 \mathrm{~K}$, and $K_{4}$ is hard to determine (present estimates for $K_{4} / D$ range from 0.005 to 0.03 ). Magnetisation and specific heat measurements indicate that, as far as the low-T dynamics is concerned, this is a good approximation for $T \leq 30 \mathrm{~K}$. The Mn- 12 molecule is of course very complicated, and early numerical attempts to establish the low-energy $H_{\text {eff }}$ did not succeed in predicting the $\mathrm{S}=10$ ground state, partly because no magnetic anisotropy was included. The difficulty of the problem can be seen by considering Fig.18(a) below, which shows the important exchange couplings, acting between the 12 different $\mathrm{Mn}$ ions in the molecule. In reality these couplings are of course anisotropic, and there will also be single-ion anisotropy terms (cf eqn (10)). Because the molecule lacks inversion symmetry, one also expects "Dzyaloshinskii-Moriya" terms, of form $V_{D M}=\sum_{<i j>} \vec{D}_{i j} \cdot\left(\vec{s}_{i} \times \vec{s}_{j}\right)$, acting between spins. Thus any serious attempt to diagonalize the full spin Hamitonian (in a Hilbert space of dimension $5^{8} 4^{4}=10^{8}$ ), with a large number of couplings which are very difficult to determine, is rather pointless. A different tactic was adopted by Tupitsyn et al, [72], following the early work of Sessoli et al. [73], in an effort to explore the region $T \leq 150 \mathrm{~K}$. It was assumed that the "diagonal" exchange couplings in Fig.18(a) are so strong that at low T, the $\mathrm{Mn}^{+3} / \mathrm{Mn}^{+4}$ ionic pair is locked into a spin-1/2 state. This reduces the problem to an 8 -spin one, with only $10^{4}$ degrees of freedom (Fig.18(b)). At this point one writes down an effective Hamiltonian for this 8-spin system, which is consistent with the known molecular symmetry, and which captures both exchange and anisotropy. The simpliest such form, which ignores any time- reversal symmetry breaking (which could come from Dzyaloshiniskii-Moriya 
interactions) can be written as follows:

$$
\hat{H}_{o}=\mathcal{J}_{1} \sum_{k=1}^{4} \vec{S}_{k} \cdot \vec{\sigma}_{k}+\mathcal{J}_{2}\left(\sum_{k=1}^{3} \vec{\sigma}_{k} \cdot \vec{\sigma}_{k+1}+\vec{\sigma}_{4} \cdot \vec{\sigma}_{1}\right)+K_{\|} \sum_{k=1}^{4} S_{k}^{z} \cdot \sigma_{k}^{z}, \quad S=2, \sigma=\frac{1}{2},
$$

where we couple 4 spin- 2 spins $\vec{S}_{k}$ and 4 spin- $1 / 2$ spins $\vec{\sigma}_{k}$ (the latter should not be confused with nuclear spins!)

FIG. 18: The structure of the exchange couplings in the $\mathrm{Mn}_{12}$, after Sessoli et al. [73]. In (a) is shown the couplings between the $12 \mathrm{Mn}$ ions; whereas in (b) we see the couplings between the 8 spins in the reduced model described in the text, where the very strongly coupled "diagonal" spin pairs (with estimated AFM coupling $J_{1} \sim-215 K$ in (a)) are coupled to form single spin- $1 / 2$ at low energy. Sessoli et al. also estimate $J_{2} \sim J_{3} \sim-85 K$ and $\left|J_{4}\right| \leq 45 K$. The renormalised couplings in (b) are discussed in the text.

We notice immediately that we have not derived this Hamiltonian. In fact if the reader is getting used to the idea of low-energy effective Hamiltonians, he/she may well be asking - from what starting point is one supposed to derive $H_{o}$ ? The answer is of course that the only reasonable starting point is one where we have some way of determing the input parameters experimentally. One might of course attempt the very ambitious task of calculating the various couplings in the 12 -spin $\mathrm{Mn}_{12} \mathrm{O}_{12}$-ac system, starting from an underlying Anderson lattice model for the molecule. From a microscopic viewpoint this certainly makes sense - we are dealing with a hydrated transition metal oxide, to which the Anderson-Mott-Hubbard ideas apply rather directly. But the problem is obvious - with no knowledge of the parameters in such a model, we can do no more than guess what superexchange couplings they might lead to, between the Mn ions. In fact, the values generally assumed for these couplings are no more than educated guesses made by the chemists.

The natural result of this line of reasoning is to treat the above 8-spin Hamiltonian as our starting point and attempt to deduce the values of the parameters $\mathcal{J}_{1}, \mathcal{J}_{2}$, and $K_{\|}$from experiments. The way this was done by Tupitsyn et al. was simple. They first calculated the magnetisation $M_{z}$ in a longitudinal field $H_{z}$, including all $10^{4}$ 
levels of the 8-spin model. The results for $M_{z}\left(H_{z}, T\right)$ were compared with a large number of experimental results on a single oriented $\mathrm{Mn}_{12}$ - ac crystal, taken at different $T$ and $H$. The parameters $\mathcal{J}_{1}, \mathcal{J}_{2}$, and $K_{\|}$were then adjusted to give the best possible fit to these experiments; it was found that this gave $\mathcal{J}_{1}=-85 \mathrm{~K}, \mathcal{J}_{2}=55 \mathrm{~K}$, and $K_{\|}=7.5 \mathrm{~K}$.

To test this model, it was then used to predict the results of a transverse magnetisation measurement, ie., the magnetisation $M_{x}\left(H_{x}, T\right)$ was calculated over a wide range of $H_{x}$ and $T$ values. These were then compared with transverse susceptibility measurements. This is a sensitive test of the theory, because the transverse field mixes together states which have little to do with each other in a longitudinal field. The results were very good for $T \leq$ $150 \mathrm{~K}$, undoubtedly because above this temperature, the "diagonal couplings" are too weak to prevent the break-up of the 4 fake spin- $1 / 2$ pairs, into their spin- 2 and spin- $3 / 2$ constituents. Thus up to $150 \mathrm{~K}$ this sort of exercise is very useful, whereas the spin-10 "giant spin" Hamiltonian breaks down seriously above 30K. With further thermodynamic measurements, eg., measurements of $C_{v}(T, H)$ (with the phonon contribution subtracted off) it should be possible to refine these models even more.

I should emphasize, however, that such calculations, although they can in principle give us a good understanding of the gross structure and origin of the eigenstates up to $\sim 100 \mathrm{~K}$, are utterly useless when it comes to understanding the tunneling. This is because, as we have already seen in Chapter 2, the tunneling matrix elements are not only exponentially smaller than the energy scale relevant to these calculations (ie., composed to anisotropy energies); they also depend exponentially on any change in these energy scales! Thus any uncertainty in our knowledge of the correct effective Hamiltonian (whether it be at the level of the giant spin $H_{o}(\vec{S})$, or at the more microscopic level just discussed) will be magnified exponentially when it is used to try and determine the tunneling matrix elements. I shall return to this point again below, in discussing the interpretation of the experiments.

(ii) Quantum Relaxation Experiments: Let us now proceed directly to the low-T experimental results on the Mn-12 and Fe-8 systems, (ie., for $T \leq 5 K$ ). We begin with Mn-12, for which many experiments have been done in recent years. The clearest of these are those by Thomas et al. [5] on a single crystal. The essential results found by the various authors were

(i) When $T>2 K$, the relaxation of an initially polarized sample of molecules was found to decay roughly exponentially (although more recent measurements by these authors also find non-exponential relaxation around the resonances at high $T$ ). The remarkable thing here was the series of resonant increases in the experimental relaxation rate $\tau^{-1}(H, T)$ around fields $n H_{1}$, where $n=0, \pm 1, \pm 2, \ldots$, and $H_{1} \sim 0.44 T$. The crucial point is that if we add a longitudinal field bias term $-g \mu_{B} S H_{z}$ to (4.1), then the resonant fields correspond precisely to "level crossings", ie., to fields at which eigenstates $|m\rangle$ of (4.1) become degenerate with eigenstates $|-m+n\rangle$ (here we define eigenstates $|m\rangle$ via the equation ${ }^{\|} H_{o}^{(2)}|m\rangle=\varepsilon_{m}^{o}|m\rangle$, where $\left.\varepsilon_{m}^{o}=-D_{m}^{2} / S\right)$. The existence of resonant increases in $\tau^{-1}$ at these fields, and a proposed explanation in terms of thermally activated resonant tunneling, was first given by Novak and Sessoli [2] (see also Barbara et al. [3).

(ii) Below $2 \mathrm{~K}$, the relaxation appears to become rather $T$-dependent in the Mn-12; moreover, recent unpublished data shows it is not at all exponential, a fact emphasized in refs. [1. [1] (but not ref. [5]).

(iii) The resonant peaks in $\tau^{-1}(H, T)$ appear to be Lorentzian around the $H_{n}$. Their half-width is rather large (roughly $0.1 T$ ).

The $\mathrm{Mn}_{12}$ experiments were followed by some very striking results [7] obtained in the "Fe" molecule. In many ways this molecule is very similar to Mn-12; a giant spin is again formed via -oxo bonds, in a typical example of antiferromagnetic superexchange in a transition metal oxide. In this system, all eight $F e$ ions are in a spin-5/2 valence state, which align again in a spin-10 ground state, which again is described to first approximation by an easy axis term ${ }^{\|} H_{o}^{(2)}$ (eg. (4.1)); in this system $D \sim 2.7 K$. The lowest transverse term which breaks rotational symmetry 
about $S_{z}$ must take the form ${ }^{\perp} H_{o}^{(2)}=-(E / S)\left[S_{x}^{2}-S_{y}^{2}\right]$ (a term disallowed in $M n_{12}$, which crystallizes in tetragonal form); just as in $M n_{12},{ }^{\perp} H_{o} / \| H_{o} \ll 1$.

The relaxation results in $\mathrm{Fe}_{8}$ molecular crystals share several features in common with those of $M n_{12}$. Again, one sees resonantly enhanced relaxation at fields $H_{n}$; but now the relaxation rate $\tau^{-1}(H, T)$ increases over 4 orders of magnitude around the zero field resonance (which, incidentally, is shifted from $H=0$ by several hundred Gauss)! A particularly valuable feature of the $F e_{8}$ system is that one may see reasonably rapid relaxation down to very low $T$; in fact, Sangregorio et al. [7] followed it down to $70 \mathrm{mK}$. Very direct evidence appears in these experiments of a crossover, below $T \sim 360-400 \mathrm{mK}$, to a regime of quantum relaxation. The relaxation $M(t)$ of the magnetisation $M$ in an initially polarised sample is completely $T$-independent below $360 \mathrm{mK}$. It is very noticeably non-exponential; in fact the authors attempted to fit it using a stretched exponential.

How we might understand these results? Before looking at the relaxation itself, I think we need some cautionary remarks concerning the use of the giant spin Hamiltonian in discussing this sort of experiment. These concern the relationship between the values of the tunneling matrix elements $\Delta_{m}$, between levels $|m\rangle$ and $|-m\rangle$ of the longitudinal part of the giant spin Hamiltonian. In what follows we will treat a matrix element like $\Delta_{10}$, coupling the 2 lowest levels of $\|_{\mathcal{H}_{0}^{(2)}}$ in $\mathrm{Mn}_{12}$ and $\mathrm{Fe}_{8}$, as an independent parameter. One can of course calculate $\Delta_{10}$ if the weak anisotropy couplings are known. Thus a 2nd-order (in $S^{+}, S^{-}$) anisotropy term ${ }^{\perp} \mathcal{H}_{o}^{(2)}=E\left(S_{x}^{2}-S_{y}^{2}\right)$ yields

$$
\Delta_{10}=\frac{4 S^{2} E^{S}(2 S-1) ! !}{(2 D)^{S-1}(2 S) ! !} \approx \frac{4 S^{3 / 2}}{\sqrt{\pi}} E(E / 2 D)^{S-1} .
$$

Using $E=-0.046 \mathrm{~K}$ for $\mathrm{Fe}_{8}$ molecules [7 then gives $\Delta_{10} \sim 10^{-9} \mathrm{~K}$ ! On the other hand the 4th-order anisotropy term ${ }^{\perp} \mathcal{H}_{o}^{(4)}=B\left(S_{+}^{4}+S_{-}^{4}\right)$ yields

$$
\Delta_{10}=\frac{\left(B S^{2}\right)^{S / 2} S^{2}(2 S) !}{(D)^{S / 2-1} 16^{[S / 4]}[S ! !]^{2}}=B S^{2}\left(B S^{2} / D\right)^{S / 2-1} \frac{(2 S) !}{S^{S-2} 16^{[S / 4]}[S ! !]^{2}} .
$$

In the $\mathrm{Mn}_{12}$ system the ${ }^{\perp} \mathcal{H}_{o}^{(2)}$ anisotropy term is absent, and the parameter $g_{4}=\left(B S^{2}\right) / D$ is rather small (estimates range from $g_{4} \approx 0.03$ down to $g_{4} \sim 0.005$. For $g_{4}=0.03$ one gets $\Delta_{10} \approx 10^{-10} \mathrm{~K}$.

From these examples we learn that even rather small higher-order anisotropy terms may determine highorder tunneling matrix elements. This is very simply because, as already noted in section 2 , a higher-order transverse coupling (eg., a coupling of 8-th order in $S^{+}, S^{-}$) appears raised to a lower power in $\Delta_{10}$ than a 2 nd- or 4 thorder coupling term, and so can play an important role even if it is much smaller than a 2nd- or 4th-order term. Consequently, in, eg., $\mathrm{Fe}_{8}, \Delta_{10}$ may be considerably larger than Eq.(131), because of such higher-order terms (up to the twentieth order in $S^{+}, S^{-}$, in fact!). They are almost impossible to obtain experimentally, since their contribution to the transitions studied by EPR spectroscopy is likely to be negligible. All I am doing here, of course, is repeating the warning made earlier about the exponential dependence of tunneling amplitudes on microscopic parameters, in a different way.

Note that as a corollary to this argument, one has to be particularly cautious in predicting the effects of the applied weak transverse field $H_{\perp}$ on $\Delta_{10}$, since this field has a contribution of order $H_{\perp}\left(H_{\perp} / D\right)^{2 S-1}$, ie., to the $2 S$-th power in the field! Essentially this means that its direct effect at low $T$ is very small- thus, the effect of any applied transverse field on $\Delta_{10}$ is negligible unless $H$ is of order Tesla. Likewise, the effect of static internal transverse fields (such as transverse hyperfine or dipolar fields when $k T \ll D$ ) on $\Delta_{10}$ is negligible.

\section{1(b) THEORETICAL INTERPRETATIONS}


To deal with relaxation we must take proper account of environmental effects. Historically the first attempt was made by Villain and co-workers, even before resonant tunneling was established [53, 54]. They considered the coupling between a giant spin, described by the Hamiltonian in equations (4.1) and (4.2), and a bath of phonons. The physics of spin-phonon couplings is well understood at the microscopic level [52], and Politi et al. [54 gave a treatment of the phonon-mediated tunneling relaxation of a giant spin $\vec{S}$, for the particular case of a single isolated $M n_{12}$ molecule. However this theory disagreed with the experiments. More recently they have extended it, and some other authors [74, 75] have given similar treatments.

In what follows I will lay considerable stress on the fact that none of these treatments is capable of explaining the low- $T$ relaxation in the Quantum Regime, for the simple reason that at these temperatures the phonons can play no role. It can be then more or less deduced from the experiments that the relaxation must be mediated by nuclear spins, which constitute the only dynamic environment remaining in the quantum regime. In this sense the low- $T$ results are a kind of "smoking gun" for the role of the spin bath in the quantum dynamics of the system. However it is clear that at higher $T$ both phonons and nuclear spins must be involved (as well as the rapidly fluctuating magnetic dipolar fields).

(i) Phonon-mediated Relaxation: Let us briefly review this theory and its results. This is useful because despite the fact that the calculations of Villain et al. [53, 54] are correct, their results disagree strongly with the experiments, particularly at low $T$ and low $H$ (ie., in the Quantum regime), and it is important to understand why.

Consider first the form of the coupling between $\underline{S}$ and phonons (parametrized, as in Chapter 2, by operators $b_{\vec{q}}, b_{\vec{q}}^{+}$, frequencies $\omega_{q} \sim c_{s} q$, and a Debye temperature $\Theta_{D}$ ). For a strongly easy-axis "Ising-type" anistropy, there is the transverse term already given previously (cf. eqtn. (52)). That the coupling should be proportional to $\Omega_{o}$, the "bounce frequency" introduced in section 2, is easily shown using instanton methods where applicable [17, but is also fairly obvious from dimensional arguments. For strongly anisotropic "Ising-like" molecules like $M n_{12}$ or $F e_{8}, \Omega_{0} \rightarrow D$ (recall that $\Omega_{0}$ is roughly the energy separation between the 2 lowest levels of the symmetric van Hemmen/Sutö Hamiltonian, and the next levels). The same result is found using standard spin-phonon theory [52].

There are of course other spin-phonon coupling terms, but before asking why this one is the most important, let us see what it does to the dynamics of $\vec{S}$. Consider 2 possible transitions between eigenstates $\mid m>$ of the longitudinal part " $H_{o}^{(2)}$ of the giant spin Hamiltonian (here $|m>=| S>,|S-1>, \ldots|-S>$ ). These are mediated simultaneously by the tunneling term ${ }^{\perp} H_{o}(s)$, and by the coupling to the phonons. Now the crucial point here is that a non-diagonal coupling like the one just given allows a transition $|m>\rightarrow|-(m \pm 1)>$, provided $\mid m>$ and $\mid-m>$ are in resonance (ie., provided $\left|\varepsilon_{m}-\varepsilon_{-m}\right| \leq \Delta_{m}$, where $\Delta_{m}$ is the tunneling matrix element between $\mid m>$ and $\mid-m>$, coming from ${ }^{\perp} H_{o}$, and $\varepsilon_{m}$ is defined by ${ }^{\|} H_{o}\left|m>=\varepsilon_{m}\right| m>$ ). This inelastic transition goes at a rate proportional to $\xi^{3}$, where $\xi=\left(\varepsilon_{m}-\varepsilon_{-m-1}\right)$ is the relevant energy difference between initial and final states, for Debye phonons. This standard result [52] comes from the available phase space for phonons. A simple calculation [54. 17] gives a non-diagonal relaxation rate

$$
\tau^{-1}(\varepsilon, T) \sim e^{-\varepsilon_{m} / k T} \frac{S^{2} \Delta_{m}^{2}}{\Theta_{D}}\left(\frac{\varepsilon}{\Theta_{D}}\right)^{3}
$$

at low T. On the other hand a diagonal coupling (ie., one not involving operators $S_{x}, S_{y}$ ) will have a much smaller available phase space. This is why the non-diagonal coupling dominates.

Suppose now that we are in the low- $T$ limit; this arises precisely when $k T \leq \Omega_{0} / 2 \pi$. The crossover occurs experimentally for $T_{c} \sim 2 K$ in $M n_{12}$, and $T_{c} \sim 0.4 K$ in $F e_{8}$. Below $T_{c}$, only the 2 lowest levels are involved. In this 
case a more accurate formula for the phonon relaxation rate is [17]:

$$
\tau^{-1}(H, T) \sim \frac{S^{2} \Delta_{10}^{2}}{\Theta_{D}}\left(\frac{\xi}{\Theta_{D}}\right) \operatorname{coth}(\xi / 2 k T)
$$

Now we immediately notice 2 things about this result. First, $\tau(H, T)$ is extremely long for low fields (ie., where the bias $\xi=\left(\varepsilon_{10}-\varepsilon_{-10}\right)$ is generated only by internal bias fields like nuclear hyperfine fields or intermolecular dipolar fields - in this case $\xi \sim 0.1-1 K$ only). Thus for $S=10$, and for the values of $\Delta_{10}$ estimated for $M n_{12}$ and $F e_{8}$ (for which $\Delta_{10}$ is almost certainly less than $10^{-8} \mathrm{~K}$, perhaps much smaller), one finds that $\tau(H, T)$ at low $T$ and low $H$ becomes longer than the Hubble time!

The 2nd thing we notice is that around $H=0$, this sort of spin-phonon theory predicts a rapid increase $\left(\sim H^{3}\right)$ of $\tau^{-1}(H)$ as $|H|$ is increased.

Unfortunately these 2 results are flatly contradicted by the experimental results, which show (for $\mathrm{Fe}_{8}$ and $\left.M n_{12}\right)$ a very sharp resonant maximum in $\tau^{-1}(H)$ around $H \sim 0$; in the middle of this the relaxation rates can be inverse seconds only (although, as already noted, the short time relaxation is far from exponential in the low $\mathrm{T}$ regime).

Thus we see that the low-T results are quite fatal to a theory which only involves a phonon environment. However, this is by no means all that one can deduce from these low- $\mathrm{T}$ results.

(ii) Nuclear Hyperfine, and Molecular Dipole fields: Suppose we now include the coupling of each molecule to (i) nuclear spins, and (ii) the magnetic dipolar fields generated by its neighbours- these are the two important couplings left in the problem.

Since, at low $T$, we have seen that phonons are irrelevant to the relaxation, let us consider the problem solely in the presence of these 2 couplings. Below $T_{c}$ the problem now reduces to an effective Hamiltonian.

$$
H=\frac{1}{2} \sum_{i j} V_{i j}^{(d)} \tau_{z}^{(i)} \tau_{z}^{(j)}+\sum_{i} \Delta_{10} \tau_{x}^{(i)}+\sum_{i k} V^{(N)}\left(\tau_{z}^{(i)}, \vec{I}_{k}\right)+H^{N N},
$$

where the first term describes the static dipolar-dipolar interactions between molecules, the second describes tunneling, the third couples magnetic molecules to nuclear spins $\left\{\vec{I}_{k}\right\}$, and the last term describes interactions between the nuclear spins. This effective Hamiltonian operates in the subspace of the two lowest levels of each molecule; we choose the basis set to be $\left|S_{z}= \pm S\right\rangle ; \tau_{z}$ and $\tau_{x}$ are Pauli matrices, and $\{i\},\{j\}$ label molecular sites. Only the longitudinal dipolar interaction appears; transverse "flip-flop" processes are exponentially small in the parameter $\left(\varepsilon_{9}-\varepsilon_{10}\right) / k T$.

Now it is essential to notice that if we also treat the hyperfine fields as static, we are faced with a really gross contradiction with experiment. As already mentioned, the bias $\xi=\left(\varepsilon_{10}-\varepsilon_{-10}\right)$ due to the combined nuclear and dipolar couplings, is not enough to allow phonons to act; moreover, the transverse fields coming from these couplings are far too small to make any noticeable change to $\Delta_{10}$. However the most crucial point is simply this: all but a very tiny fraction of molecules are blocked from any transitions at all, because $\xi \gg \Delta_{10}$ for almost all molecules. From this point of view it is quite incredible that there is any relaxation at all in the low- $T$ limit; recall that the energy barriers in these experiments are very large compared to $k T$ (at the lowest temperatures in the Fe- 8 experiments, $k T$ was nearly 500 times less than the barrier height, and some 100 times smaller than the energy $\sim 2 \mathcal{D}$ required to excite any but the 2 lowest levels of each molecule!). Nevertheless one sees rapid relaxation - why?

I have very recently argued, in a paper with N.V. Prokof'ev [76], that the explanation is more or less obvious, and can in fact be deduced from the experiments. The basic point is that no relaxation at all can proceed unless we 
give the environment some dynamics; as we have just seen, a static Hamiltonian won't work. But at $70 m K$ there is only one source of such dynamics, as we have already seen in Chapters 2 and 3; it comes from the transverse "spin diffusion" fluctuations in the hyperfine field, which have a typical frequency $T_{2}^{-1} \sim 10-100 \mathrm{kHz}$. Unlike phonons, or intermolecular dipolar flip-flop transitions, or nuclear $T_{1}$ transitions (all of which disappear in the low-T limit, and are exponentially small in the ratio $\mathcal{D} / k T$ ), the nuclear $T_{2}$ dynamics persists down to temperatures in the $n K$ range, and is fairly independent of $T$ in the present experimental temperature range.

We have already seen in Chapter 3 what these rapid hyperfine fluctuations can do. For the present problem, the hyperfine coupling is sufficiently weak that no nuclear spins are likely to be flipped when $\vec{S}$ flips. Thus a molecule near resonance (ie., where $\xi$ is small), will relax incoherently between states $\mid 10>$ and $\mid-10>($ or vice versa) at a rate given by

$$
\begin{aligned}
\tau_{N}^{-1}(\xi) & \approx \tau_{0}^{-1} e^{-|\xi| / \xi_{o}} . \\
\tau_{0}^{-1} & \approx \frac{2 \Delta_{10}^{2}}{\pi^{1 / 2} \Gamma_{2}} .
\end{aligned}
$$

where we have assumed a fluctuating bias field $\xi(t)=\xi+\delta \xi(t)$, for which the fluctuations $\delta \xi(t)$ extend over a range $\xi_{o} \sim T_{2}^{-1}$ (compare eqtn.(127)). The basic physics is simple; not only do the fluctuations greatly widen the "resonance window" width (from $\Delta_{10}$ to $\xi_{o}$, an increase of several orders of magnitude), they also allow the transitions to be inelastic (and therefore to cause irreversible relaxation). Thus, at very short times, molecules with a bias within $\xi_{o}$ of zero are sucked into the resonance window, where they undergo irreversible inelastic relaxation.

At this point the long-range dipolar fields come in. Once a fraction $\xi_{o} / W$ of molecules has relaxed via this mechanism (where $W \sim 0.1-1 K$ is the total spread in longitudinal bias fields around the sample), the adjustment of the long-range dipolar fields caused by these transitions is quite enough to bring more molecules into the resonance window. At first glance this seems like a very complex problem; however, to treat it is actually quite straightforward, using standard kinetic theory methods.

We begin by defining a normalised 1-molecule distribution function $P_{\alpha}(\xi, \vec{r} ; t)$, with $\sum_{\alpha} \int d \xi \int d \vec{r} P_{\alpha}(\xi, \vec{r} ; t)=$ 1. It gives the probability of finding a molecule at position $\vec{r}$, with polarisation $\alpha= \pm 1$ (ie., in state $\left|S_{z}= \pm S\right\rangle$ ), having a bias energy $\xi$, at time $t$. Molecules having bias energy $\xi$ undergo transitions between $\left|S_{z}=S\right\rangle$ and $\left|S_{z}=-S\right\rangle$ at a rate given by (136). Flipping these molecules then changes the dipolar fields acting on the whole ensemble, bringing more molecules into near (or away from) resonance, and leading to a self-consistent evolution of $P_{\alpha}(\xi)$ in time. The general solution of this problem requires a kinetic equation for $P_{\alpha}(\xi, \vec{r} ; t)$.

To derive a kinetic equation for $P_{\alpha}(\xi, \vec{r} ; t)$, we again assume that dipolar and hyperfine fields are frozen (apart from the nuclear $T_{2}$ fluctuations just discussed), unless a molecule flips. All kinetics then come from these flips, along with the resulting adjustment of the dipolar field. We may then derive a kinetic equation in the usual way, by considering the change in $P_{\alpha}$ in a time $\delta t$, caused by molecular flips, at the rate $\tau_{N}^{-1}(\xi)$, around the sample. This yields

$$
\begin{aligned}
\dot{P}_{\alpha}(\xi, \vec{r})= & -\tau_{N}^{-1}(\xi)\left[P_{\alpha}(\xi, \vec{r})-P_{-\alpha}(\xi, \vec{r})\right] \\
& -\sum_{\alpha^{\prime}} \int \frac{d \vec{r}^{\prime}}{\Omega_{0}} \int \frac{d \xi^{\prime}}{\tau_{N}\left(\xi^{\prime}\right)}\left[P_{\alpha \alpha^{\prime}}^{(2)}\left(\xi, \xi^{\prime} ; \vec{r}, \vec{r}^{\prime}\right)-P_{\alpha \alpha^{\prime}}^{(2)}\left(\xi-\alpha \alpha^{\prime} V_{D}\left(\vec{r}-\vec{r}^{\prime}\right), \xi^{\prime} ; \vec{r}, \vec{r}^{\prime}\right)\right]
\end{aligned}
$$

where $P_{\alpha \alpha^{\prime}}^{(2)}\left(\xi, \xi^{\prime} ; \vec{r}, \vec{r}^{\prime} ; t\right)$ is the two-molecule distribution, giving the normalized joint probability of finding a molecule at site $\vec{r}$, in state $|\alpha\rangle$ and in a bias $\xi$, whilst another is at $\vec{r}^{\prime}$, in state $\left|\alpha^{\prime}\right\rangle$, and in a bias $\xi^{\prime}$. $P^{(2)}$ is linked to higher multi-molecule distributions by a BBGKY-like hierarchy of equations. The first term on the right-hand side of 
(138) describes the local tunneling relaxation, and the second non-local term (analogous to a collision integral) comes from the change in the dipolar field at $\vec{r}$, caused by a molecular flip at $\vec{r}^{\prime}$; the dipolar interaction $V_{D}(\vec{r})=$ $E_{D}\left[1-3 \cos ^{2} \theta\right] \Omega_{0} / r^{3}$, where $\Omega_{0}$ is the volume of the unit molecular cell, and $\int d \vec{r}^{\prime}$ integrates over the sample volume.

As discussed in our paper, with this kinetic equation we can make a number of very clear predictions concerning the low-T relaxation in these magnetic molecular crystals. These include (i) The prediction that an initially polarized sample will, at short times, relax according to a universal $\sqrt{t}$ law (where $t$ is the elapsed time); the sample magnetisation will obey

$$
M(t)=\left[1-\left(t / \tau_{\text {short }}\right)^{1 / 2}\right] M_{o}
$$

where $M_{o}$ is the saturated magnetisation, provided $1-\left(M / M_{o}\right) \ll 1$. (ii) The timescale $\tau_{\text {short }}$ depends radically on the sample shape. For an ellipsoidal sample one finds

$$
\tau_{\text {short }}^{-1}=\frac{\xi_{0}}{E_{D} \tau_{0}} \frac{32 \pi}{3^{5 / 2}\left(c^{2}+16 \pi^{2} / 3^{5}\right)} .
$$

where the constant $c$ is given by magnetostatic theory; for a prolate spheroid, one finds

$$
c=(2 \pi / 3)\left[a^{4}+a^{2}-3 a \sqrt{a^{2}-1} \ln \left(a+\sqrt{a^{2}-1}\right)-2\right] /\left(a^{2}-1\right)^{2}
$$

where $a$ is the ratio of the longitudinal axis to its perpendicular; analytic formulas can be found for any ellipsoid.

The analytic simplicity of these results arises because in the short-time limit, $P^{(2)}\left(\xi, \xi^{\prime} ; \vec{r}, \vec{r}^{\prime} ; t\right)$ is factorizable, and because for an ellipsoid, the initial demagnetisation field is homogenous (a standard result of magnetostatic theory). For a sample of arbitrary shape, we still get $\sqrt{t}$ relaxation, but in this case $\tau_{\text {short }}$ above is replaced by

$$
\left(\tau_{\text {short }}^{(\text {inh })}\right)^{-1} \sim \xi_{0} N(0) \tau_{\text {short }}^{-1},
$$

where $N(0)=\int d \vec{r} \sum_{\alpha} P_{\alpha}(\xi=0, \vec{r} ; t=0)$ is the initial "density of states" for the dipolar field distribution, integrated over the whole sample, at bias $\xi=0$; typically $N(0) \sim 1 / E_{D m}$, where $E_{D m}$ is the average demagnetization field.

Notice that the prediction for these timescales shows that they depend essentially on both the nuclear fluctuation timescale $T_{2}$ and on the sample shape. Once the condition $1-\left(M / M_{o}\right) \ll 1$ is relaxed, we must worry about the non-factorisability of $P^{(2)}$, and analytic calculations are no longer so easy. However it is easy to both verify the above results for short times, and to extend them to longer times, using Monte Carlo (MC) simulations of the relaxation in samples of various shapes [76]. It is also interesting to see how the 1- molecule distribution function $P_{\alpha}(\xi, \vec{r} ; t)$ itself relaxes; this is shown in Fig.19, at short times, for a homogeneous spherical sample.

Why is the short-time behaviour so simple (and universal)? Essentially because we have a small parameter (the fraction of flipped spins). These spins behave like a set of dilute dipoles, creating a solution to the kinetic equation which is in fact an old friend to anyone who has worked in NMR. Consider first for simplicity the case of an ellipsoidal sample, so that the initial field is homogeneous. Then at short times the solution to our kinetic equation, if the normalised magnetisation at $t=0$ is unity, just becomes

$$
\dot{M}(t)=-M(t) \frac{2}{\tau_{0}} \int d \xi e^{-|\xi| / \xi_{0}} \frac{\Gamma_{d}(t) / \pi}{[\xi-E(t)]^{2}+\Gamma_{d}^{2}(t)} .
$$

where the field distribution in an ellipsoidal sample is nothing but a Lorentzian distribution (up to a high energy cut-off $E_{D}$ ) related to that found by Anderson for dilute static dipoles:

$$
P_{\alpha}(\xi)=\frac{1+\alpha M(t)}{2} \frac{\Gamma_{d}(t) / \pi}{[\xi-\alpha E(t)]^{2}+\Gamma_{d}^{2}(t)} ;
$$




$$
\begin{aligned}
\Gamma_{d}(t) & =\frac{4 \pi^{2}}{3^{5 / 2}} E_{D}(1-M(t)) ; \\
E(t) & =c E_{D}(1-M(t)),
\end{aligned}
$$

where $c$ is the sample shape dependent coefficient defined above, and $E(t)$ is the time-dependent internal field (assuming here that the external field is zero).

FIG. 19: Monte-Carlo (MC) simulation of the time evolution of the time-dependent density of states $N_{\alpha}(\xi, t)=\int d \vec{r} P_{\alpha}(\vec{r}, \xi ; t)$ of the unflipped spins in a spherical sample of diameter 50 sites, with spins arranged in a cubic lattice. Energy and density of states use units where $\xi_{0}=1$ and $E_{D}=20$, and results are shown for $\alpha=+1$ at time $t=0$ (for an initially polarized sample) and for $\alpha=-1$ at $t=0.1 \tau_{0}$. The Lorentzian shape is distorted by lattice effects at high energy (giving the hump at $E=-15$ ). The noise, and the finite width of the $\delta$ function at $t=0$, are finite size effects.

This explains the universality of the $\sqrt{t}$ behaviour for an ellipsoidal sample (and note the distinctive and strong dependence of $\tau_{\text {short }}$ on the sample shape- this should be testable experimentally). But what about samples of arbitrary shape? Then the problem becomes essentially inhomogeneous. We thus return to the kinetic equation (138), and notice that if the demagnetisation varies on a length scale much greater than the average distance between flipped spins, then (143) is simply modified to

$$
\dot{M}(\vec{r}, t)=-M(\vec{r}, t) \frac{2}{\tau_{0}} \int \frac{d \xi}{\pi} \frac{\Gamma_{d}(\vec{r}, t) e^{-|\xi| / \xi_{0}}}{[\xi-E(\vec{r}, t)]^{2}+\Gamma_{d}^{2}(\vec{r}, t)} .
$$

where $\Gamma_{d}(\vec{r}, t)$ and $E(\vec{r}, t)$ are defined in terms of $M(\vec{r}, t)$ analogously to $(144)$ and(145); the solution is then identical to (139) except that $\tau_{\text {short }}$ is modified to $\tau_{\text {short }}^{(i n h)}$. You can think about this in a geometrical way. In an ellipsoidal sample, with initially uniform field, the molecules are either initially in the "resonance window" (of width $1 / \xi_{o}$ ) or they are not. If they are, then the rapid decrease in the instantaneous relaxation rate, giving the square root 
form, comes about because the molecules are being pushed away from resonance as the inhomogeneous Lorentzian fields develop. Only a Lorentzian shape gives a $\sqrt{t}$ relaxation; and when the flipped spins become dense enough, the Lorentzian form must break down (in fact it probably becomes more gaussian, at least for intermediate times). Now turning to a sample of arbitrary shape, one should imagine the surface inside the sample which is the locus of points where the total field (ie., the sum of external field $H_{o}$ and the internal field $E(\vec{r}, t)$ ) is zero. When the sample starts relaxing, you can imagine that an expanding region around this surface becomes involved in the relaxation, with molecules inside it being either brought into or forced out of resonance. In a sample of complicated shape (particularly with edges and corners) this surface (and the related density of states) will be complicated, with van Hove singularities of various kinds in $N(\xi)$ (in principle classifiable using Morse theory). However, this does not in any way affect the way in which the function $N_{-}(\xi, t)$, integrated over the sample, develops a Lorentzian shape around $\xi=0$ at short times.

I strongly emphasize that as soon as we have a reasonably large concentration of flipped molecules (roughly 10 to 15 per cent, according to the MC simulations), this analysis must begin to break down, because the bias field distribution, due to the flipped spins, will stop being Lorentzian. Moreover, we also expect the assumption of factorisation of the 2-molecule distribution function to break down, and the problem then apparently becomes intractable- in fact we have a kind of Quantum Spin Glass problem. We expect a complicated crossover to a long time behaviour which shows "ageing" behaviour, and whose form seems very difficult to predict.

However, one can also make analytical predictions for long times under a slightly different circumstance, viz., if the sample is first depolarized at high $T$, to a value $M / M_{o} \ll 1$, and the cooled to the low-T quantum regime. In this case we also have factorizability of the 2-particle distribution function; and in fact another analytic solution for the homogeneous (ie., ellipsoidal) case can be found from (138), when $M \ll 1$ and $P_{\alpha \alpha^{\prime}}^{(2)}\left(\xi, \xi^{\prime} ; \vec{r}, \vec{r}^{\prime}\right)=P_{\alpha}(\xi) P_{\alpha^{\prime}}\left(\xi^{\prime}\right)$. One finds exponential relaxation, at a rate

$$
\tau_{\text {long }}-1 \approx \frac{2 \xi_{0}}{E_{\max } \tau_{0}\left[1+\kappa \ln \left(E_{\max } / \pi \xi_{0}\right)\right]},
$$

where $\kappa \sim 1$ is a numerical coefficient, and $E_{\max }$ is the spread in dipolar fields in this nearly depolarized limit.

It will be very interesting to see if these low-T predictions are confirmed. I emphasize that the low- $T$, low- $H$ limit is crucial here - in this case the predictions can more or less be deduced directly, along the lines just described. In my personal opinion, confirmation of the $\sqrt{t}$ law, and the dependence of $\tau_{\text {short }}$ on $T_{2}$ and on sample shape, would give very powerful evidence for the nuclear relaxation mechanism discussed. In any case, we see here a very nice example of the role a nuclear spin bath can play in tunneling dynamics. The essential result which the spin bath theory gave us is the formula for $\tau_{N}^{-1}(\xi)$ in eqtn (137) (and this result is just a special case of eqtn (126)).

What more theoretical work can be done in this low- $T$ limit? The first obvious thing is to extend this kind of theory to much higher fields (both longitudinal, which will bring level $\mid 10>$ into resonance successively with levels $|9>| 8>$,, etc; and transverse fields, which will slowly reduce the total barrier height, as well as slowly changing the tunneling matrix elements. The effect of longitudinal fields will not be simple - even in the very low- $\mathrm{T}$ limit, the resonant tunneling between $\mid 10>$ and $\mid-9>$ will be quite different from that between $\mid 10>$ and $\mid-10>$, since both spontaneously-emitted phonons and dipolar processes will play a role.

Another unsolved problem of great interest at low $T$ is the effect of an AC field on the nanomolecular dynamics. A number of papers have already been written on this, essentially treating the problem as a simple Landau-Zener problem, involving only the giant spin levels and the AC field. However I should emphasize strongly that the Landau-Zener transitions, when they take place, are doing so in the presence not only of the applied AC field, but also of the much more rapidly fluctuating nuclear fields, even at the very lowest temperatures (and of 
course at higher $T$, they have to contend with the large and wildly fluctuating intermolecular dipolar fields!). The experiments have already shown a very rich behaviour (see particularly recent work of Novak et al., [77]), which depend strongly on $H$ and $T$.

(iii) Thermally-Activated Regime: I concentrated on the low- $T$, low- $H$ regime above, in discussing the experiments, simply because their interpretation in this regime was straightforward (and provides clear evidence for nuclear spin effects). The experiments at temperatures above $T_{c}$ are more complex to interpret (because of the large number of physical processes which are simultaneously involved), but are also of considerable interest.

It is always a good idea, in trying to understand a set of experiments, to see what happens in regimes where the theory is simple - and this often means looking for "limiting cases", in which one or more parameters are very small (or very large), so that some processes dominate and others are irrelevant. We have just seen this in the low- $T$ limit (where all dynamics except transverse nuclear spin fluctuations are frozen out). Is there any analogous simplifying limit at higher $T$ ?

At first glance this is not obvious - at higher $T$ many more giant spin levels are involved, as are now the phonons, and quite a number of recent papers have dealt with processes involving phonon-mediated transitions between these levels. For reasons quite obscure to me, most of these papers treat both the nuclear hyperfine and the molecular dipoles fields as static, when in fact they are both fluctuating rather violently and rapidly in time. Thus the relaxation, at first, seems very messy.

In fact, however, things are simple for the following reasons:

(i) The timescales involved in the various physical processes are much shorter than the experimental relaxation timescales (and moreover this is exactly what should have been expected once $T$ is considerably greater than $T_{c}$.

(ii) The competition between the experimentally increasing tunneling matrix elements, and the experimentally decreasing thermal occupations, as one rises up through higher and higher giant spin levels, means that at given values of $H$ and $T$, one transition will dominate the tunneling relaxation.

There is nothing particularly radical about these circumstances - in fact they are perfectly normal. Let us see how they arise.

We note that first that thermal equilibration between levels on the same side of the energy barrier is very fast, over timescales $\sim \mu$ secs: this is easily shown if we assume spin-phonon coupling strengths. Such processes do not involve tunneling. We also notice that, in contrast to the low- $T$ case, dipolar "flip-flop" processes now occur very rapidly - in fact at a rate typically even faster than spin-phonon transitions. Thus, for small $H$, transitions like $|9\rangle|-10\rangle \rightarrow|10\rangle|-9\rangle$ (or vice-versa) happen at a frequency exceeding $10^{6} \mathrm{~Hz}$ once we are well out of the quantum regime.

These 2 processes are the only ones which can cause transitions between 2 giant spin levels on the same side of the barrier - they were both frozen at low $T$, but their rate increases exponentially fast as $T$ rises. The now very rapid dipolar fluctuations also completely change the nuclear spin dynamics; the longitudinal nuclear relaxation rate $T^{-1}$ rises with the dipolar flip-flop rate, since the latter drives the former. This is important, because it means that the bias energy $\xi=g \mu_{B} H S_{z}$ acting on each molecule is fluctuating very fast (because of the fluctuations of both $\vec{H}_{d i p}$ and $\vec{H}_{h y p}$ ) over a fairly large range of energies (for $F e_{8}, \vec{H}_{d i p} \gg \vec{H}_{H y p}$, and $\xi$ fluctuates over a range $\sim 0.5 K$, whereas for for $\mathrm{Mn}_{12}$, the hyperfine field is much larger, indeed not much smaller than the dipolar fields, so that the fluctuations in $\xi$ may well be some 50 per cent larger). Notice that although we do not know how fast $T_{1}$ is (it has not been measured for any of these molecules at the relevant temperatures), we do expect it to have roughly the same $T$-dependence as the dipolar flip-flop rate; and we expect $T_{1} \ll \tau_{\exp }$ (where $\tau_{\exp }$ is the experimental relaxation 
rate), once we are well inside the thermally-activated regime.

Thus the picture that energies in the thermally activated regime is one where molecules cycle between giant spin states on the same side of the barrier, and between all the nuclear multiplets available for a given giant spin state $|m\rangle$. Inelastic tunneling transitions are much slower. What then controls the tunneling rate in this regime?

The answer is startlingly simple. The transitions on one side of the barrier occur so quickly that they disappear completely from the physics - their only role is to keep the molecules on one side of the barrier in a state of quasi-equilibrium, in which the probability of occupying a state of energy $\epsilon$ is just $Z^{-1} e^{-\epsilon / k T}$, where $Z$ is the total partition function. Under these circumstances the relaxation rate is controlled only by the coupling to phonons, and we expect exponential relaxation!

Let me stress again that there is nothing in this conclusion that will surprise many - in fact a number of papers have already simply assumed the existence of quasi-equilibrium thermal populations on each side of the barrier, in attempting to calculate a relaxation rate [1. However this assumption obviously needs to be justified, particularly as we know that the low- $T$ relaxation is predicted to be non-exponential! More generally, as emphasized some time ago [17], the crossover to exponential relaxation will occur on a timescale roughly equal to $T_{1}$, and so in the region of temperature where one sees crossover from quantum to thermally activated behaviour (ie., for $T \sim \mathcal{D} / 2 \pi$ ), one may even expect to see $\sqrt{t}$ relaxation for $t \ll T_{1}$, with a crossover to exponential relaxation for $t \gg T_{1}$. Experimental measurements of $T_{1}$ would be very useful here!

However a cautionary note. It is not entirely obvious that one will always expect to see exponential relaxation in the experiments, even at high $T$. The reason for this is very simple. In a typical relaxation experiment, as we have already seen at low $T$, the total field (internal field $E(\vec{r}, t)$ plus external applied field $\vec{H}_{o}$ ) changes in time as the system relaxes; moreover, so does the distribution function $P_{\alpha}(s, \vec{r}, ; t)$, and hence the density of states $N(\xi ; t)$ available for transitions. Consequently, in the course of the relaxation, the relaxation rate $\tau^{-1}(H)$ must become a function of time - essentially we are sweeping through regions of different relaxation rate. Thus the high- $T$ relaxation is probably going to be rather complex (and indeed the various experiments do not obviously agree in this regime).

\subsection{Macroscopic Quantum Coherence?}

Ever since the well-known early discussions of Leggett et al. 48, 41, 78, on the possibility of superpositions of "macroscopically distinguishable" quantum states in SQUIDs, there has been intense interest in its experimental realisation. Discussions of this are usually framed in the context of "Macroscopic Quantum Coherence" between 2 low energy states; and following Leggett (see his chapter in this book), most of these discussions have been in the context of the spin-boson model.

Despite a number of attempts to find Macroscopic Quantum Coherence (MQC) in superconducting systems, no results of this kind have yet been reported. In section 3.4(b) above, I already discussed why I think that the observation of MQC in superconductors is going to be very difficult, because of the nuclear bath effects. However in the different field of magnetism, a rather dramatic claim for the discovery of MQC has been made by the group of Awschalom et al. [8, 9], in a series of papers going back to 1992. There has also been strong opposition to these claims from various quarters, most notably in the work of A. Garg [79].

I don't think this issue is yet settled, but it is clearly of interest; the purpose of the present sub-section is simply to present the results, and to explain the role of the nuclear spin bath in the problem.

\section{2(a) EXPERIMENTAL RESULTS on FERRITIN MACROMOLECULES}

The series of experiments reported by Awschalom et al. actually began with results 80] on arrays of Iron 
pentacarbonyl $\left(\mathrm{FeCO}_{5}\right)$ ellipsoidal grains, of rather large size. The AC susceptibility absorption was sharply peaked in these experiments, at very low frequencies (ranging from $\omega_{o} \sim 60 \mathrm{~Hz}$ for grains of size $150 \AA \times 700 \AA$, up to $\omega_{o} \sim$ $400 \mathrm{~Hz}$ for grains of size $150 \AA \times 380 \AA$ ). Curiously, it was found that $\omega_{0}$ varied roughly exponentially with the volume of the particles (decreasing with larger volume) in a way that would be expected if the particle magnetisation was tunneling coherently between 2 orientations, or else being driven by the AC field between 2 eigenstates, which themselves are superpositions of 2 "semiclassical" states $\mid \Uparrow$ and $\mid \Downarrow$ (the particles are uniaxial).

Nevertheless, as recognized by these authors, this interpretation is untenable, principally because the energy scale $\omega_{o}$ is so small (a frequency $400 \mathrm{~Hz}$ is equivalent to a temperature $\sim 8 \times 10^{-8} \mathrm{~K}$ ). For all the grains to show such a coherent response would require that any external bias field, acting on the grains, would be so small that it would split the degenerate states by a bias energy $\xi$ considerably less than $\omega_{o}$. This is of course impossible; apart from anything else the dipolar magnetic interaction between the grains is at least 5 orders of magnitude larger than $\omega_{o}$, for even the smallest grain size! In fact, to this date I am aware of no explanation offered for these results.

However in 1992 experiments done on frozen solutions of ferritin molecules in the protein apoferritin were reported [8]. Ferritin is a very interesting molecule, found naturally in all eukaryotic cells; it is of roughly spherical shape, and diameter $\sim 80 \AA$. The core contains some $4500 \mathrm{Fe}^{3+}$ ions (spin 5/2), and is protected from the outside world by a "cage" of apoferritin protein molecules.

In bulk the ferrihydrite would order antiferromagnetically (giving a Néel vector for this system of magnitude $\left.N=|\vec{N}| \sim 18,000 \mu_{B}\right)$. In reality of course the molecules possess a surface moment, which must certainly vary from one molecule to another - in their first paper Awschalom et al. [8] estimated this moment by various means, yielding values ranging from $217 \mu_{B}$ to $640 \mu_{B}$. The main result of this experiment, like the earlier $\mathrm{FeCo}_{5}$ one, was a sharp resonance in $\chi^{\prime \prime}(\omega)$ (the $\mathrm{AC}$ absorption). However this time the frequency was $\sim 940 k H z$ (ie., an energy $\left.\sim 45 \mu K\right)$. This was only seen in strongly diluted (1000:1) solutions.

More recently this group has done similar experiments on artificially engineered samples of ferritin molecules with smaller core sizes, and again they saw $\omega_{o}$ rise as a roughly experimental function of the inverse particle size []. One should also notice that no resonance was found in experiments on an apoferritin sample containing no ferritin, and that increased dilution of the sample tended to sharpen the absorption lines.

In contrast to the $\mathrm{FeCO}_{5}$ results, Awschalom et al. believe that the ferritin results provide evidence for the resonant absorption of EM waves at a frequency $\omega_{o}$ corresponding to the tunneling matrix element $\Delta_{o}$, between Néel states $|\uparrow\rangle$ and $|\downarrow\rangle$. This belief is partly based on the estimated value for the anisotropy field, which is not incompatible with a tunneling matrix element of this size.

A large number of objections have been raised to this straightforward interpretation. These include (i) the existence of randomly varying dipolar couplings between the molecules (the molecules are not oriented) [37] (ii) the field dependence of the resonance frequency and linewidth [79] (iii) the power absorption in the experiments; [79] (iv) a claimed contradiction with high- $T$ blocking [81]; and (v) nuclear spin effects [14, 16, 40]. Many of these queries will probably only be definitively settled if and when other experimental groups carry out an independent check on the experiments. I will make no attempt to address all of the issues, and refer the reader to the literature. Instead, and in the context of this chapter, we will look at the single question of the the effect of nuclear spins on the coherence.

\section{2(b) NUCLEAR SPIN EFFECTS on MQC in FERRITIN}

To investigate the effects of the nuclear bath (and other environmental spins) on the dynamics of the ferritin molecules, we need a realistic effective Hamiltonian. The problem is analogous to the SQUID problem we already looked at, and it is pedagogically useful because it deals with an antiferromagnetic system. We begin with a single ferritin molecule, for which the biaxial Hamiltonian (9) is usually used (with the understanding that $\vec{S}$ now represents 
the Neél vector, and that the extra surface moment will in reality complicate things- the following is not meant to be a complete study). Awschalom et al. [8, 9] give a value 1.72 Tesla (in field units) for $\left(K_{\|} K_{\perp}\right)^{1 / 2}$, so we will take a small oscillation frequency $\Omega_{o} \sim 40 G H z$ as a rough estimate. If we also suppose that the resonance at frequency $\omega_{o}$ represents resonant tunneling, then $\Delta_{o} \sim 1 M H z$ for the sample of naturally occurring ferritin.

¿From these numbers we immediately see that topological decoherence effects will be very small; with roughly $100 \mathrm{Fe}^{57}$ nuclei in the sample (some 2 per cent of all the nuclei have a spin), and a hyperfine coupling $\omega_{k} \sim 50-60 \mathrm{MHz}$, we get $\alpha_{k} \sim 2 \times 10^{-3}$, and so the mean number of nuclear spins co-flipping with the ferritin is $\lambda \sim 4 \times 10^{-5}$. However as noted in Prokof'ev and Stamp [14, 16], and discussed at some length later by Garg [40], the degeneracy blocking effects are very severe; the spread $E_{o}$ in the nuclear multiplet around each ferritin level is roughly $500-600 \mathrm{MHz}$, and so the mean bias is some 600 times greater than $\Delta$ (the contrary claim of Levine and Howard 82 was later retracted 83]). There will also be an effect of orthogonality blocking, whose effect is very hard to quantify; it will come from the surface moments on the ferritin, and even more from the surface interactions with the apoferritin (particularly between surface moments and any paramagnetic spins in the apoferritin; this was described as an effect of "loose spins" in the early papers [14). The ensuing random fields acting on the ferritin moment mean that the initial and final states of the Neél vector will not be exactly antiparallel.

If we ignore degeneracy blocking, and also ignore the nuclear spin dynamics, then the situation does not in fact look too bad for the experiments, at least at first glance. This is because the lineshape in a situation of pure degeneracy blocking (already shown in Fig.XXX), is rahter sharply peaked, and so resembles the experiments. There is certainly a problem of power absorption, but as noted by Awschalom et al., this is very hard to quantify [79, 31. I ignore here the other difficulties mentioned above (points (i)-(iv)).

However there is also the problem of the nuclear spin dynamics at low $T$; moreover, as we have seen already in the context of the magnetic molecules, this will drive a time-dependent change in the dipolar field distribution in the sample (which presumably starts off being pretty much Lorentzian in the sample of dilute and randomly-ordered molecules). However there is one big difference here from the case of $\mathrm{Mn}_{12}$ and $\mathrm{Fe}_{8}$ discusssed earlier. This is that in all of the experiments done on ferritin so far, $\Delta_{o}$ is bigger than the inverse timescale $T_{2}^{-1}$ of the nuclear fluctuations. This changes things a great deal- now the bath fluctuations are slow and thus have a much smaller effect on coherent tunneling.

One could go on and discuss this problem in quantitative detail. However I will not for the following simple reason. This is that none of the calculations described here (or those appearing in the literature) have yet addressed what is the key theoretical problem in the interpretation of the ferritin experiments, which is the AC response of a central spin, coupled to a spin bath, when the driving field is fast. This is nothing but the Landau-Zener problem for a system coupled to a spin bath, and it is not yet solved! I would stress that the solution to this problem is not at al obvious- indeed with a finite spin bath it is clear that under many circumstances a response function (in the strict sense of linear response) does not even exist! Thus, since whereof one cannot speak..

\subsection{Acknowledgements}

Much of the work of my own described herein was done with Drs. M. Dube' and N.V. Prokof'ev, whom I thank for innumerable discussions. At the Seattle workshop I also enjoyed very useful discussions with Drs. P. Ao, G. Bertsch, O. Bohigas, A. Bulgac, H. Grabert, P. Hanggi, P. Leboeuf, A.J. Leggett, A. Lopez-Martens, C. Sa de Melo, B. Spivak, D.J. Thouless, S. Tomsovic, and J. Treiner, although not all of the subjects we discussed appear here! I thank the Institute for Nuclear Theory (Seattle), the Canadian Institute for Advanced Research, NSERC 
Canada, and the Laboratoire de Champs Magnetiques Intenses (Grenoble), for support during the time this chapter was written. 


\section{References}

[1] C. Paulsen and J.G. Park, in "Quantum Tunneling of Magnetisation-QTM'94" (ed. L. Gunther and B. Barbara), Kluwer publishing, pp. 189-207 (1995).

[2] M. Novak and R. Sessoli, pp. 171-188 in ref. 1.

[3] B. Barbara et al., J. Mag. Magn. Mat. 140-144, 1825 (1995).

[4] J.R. Friedman et al., Phys. Rev. Lett., 76, 3830-3833 (1996).

[5] L. Thomas et al., Nature 383, 145-147 (1996).

[6] J.M. Hernandez et al., Europhys. Lett., 35, 301-306 (1996).

[7] C. Sangregorio, T. Ohm, C. Paulsen, R. Sessoli, and D. Gatteschi, Phys. Rev. Lett., 78, 4645 (1997).

[8] D.D. Awschalom et al., Science 258, 414 (1992), and refs. therein.

[9] S. Gider et al., Science 268, 77 (1995)

[10] K. Hong, N. Giordano, Europhys. Lett. 36, 147 (1996), and refs. therein.

[11] W. Wernsdorfer et al., Phys. Rev. Lett. 78, 1791 (1997); and May 1997 preprint.

[12] R.P. Feynman, F.L. Vernon, Ann. Phys. 24, 118 (1963)

[13] A.O. Caldeira, A.J. Leggett, Ann. Phys. 149, 374 (1983)

[14] N.V. Prokof'ev, P.C.E. Stamp, J. Phys. CM 5, L663 (1993)

[15] P.C.E. Stamp, Physica B 197, 133 (1994) [Proc. LT-20, Aug. 1993]. See also P.C.E. Stamp, UBC preprint (Sept. 1992, unpublished), and Nature 359, 365 (1992); and ref. [22] below.

[16] N.V. Prokof'ev, P.C.E. Stamp, pp. 347-371 in ref. 1

[17] N.V. Prokof'ev, P.C.E. Stamp, J. Low Temp. Phys. 104, 143 (1996)

[18] N.V. Prokof'ev, P.C.E. Stamp, Rep. Prog. Phys. (to be published)

[19] A. Shimshoni, Y. Gefen, Ann. Phys. 210, 16 (1991)

[20] A.O. Caldeira, A.H.Castro-Neto, T. Oliveira de Carvalho, Phys. Rev. B48, 13974 (1993).

[21] S. Sachdev, Physics World 7, 25 (1994), and refs. therein; R.N. Bhatt, S. Sachdev, J. Appl. Phys. ; C.T. Muruyama, W.G. Clark, J. Sanny, Phys. Rev. B29, 6063 (1984).

[22] P.C.E. Stamp, Phys. Rev. Lett. 61, 2905 (1988)

[23] N. Nagaosa, A. Furusaki, M. Sigrist, H. Fukuyama, J. Phys. Soc. Jap. 65, 3724 (1996)

[24] M. Yamanaka, N. Nagaosa, P.C.E. Stamp, to be published. 
[25] D.L. Hill, J.A. Wheeler, Phys. Rev. 89, 1102 (1953)

[26] A. Bulgac, G. Do Dang, D. Kusnezov, Phys. Rev. E54, 3468 (1996), and Ann. Phys. 242, 191 (1995); and refs. therein.

[27] K.G. Wilson, Rev. Mod. Phys. 47, 773 (1975).

[28] M. Dube, P.C.E. Stamp, J. Low. Temp. Phys. (accepted Aug. 1997); /cond-mat 9708191

[29] J.L. van Hemmen, A. Suto, Europhys. Lett. 1, 481 (1986), and Physica B141, 37 (1986); J.L. van Hemmen, W.F. Wreszinski, Comm. Math. Phys. 119, 213 (1988); and J.L. van Hemmen, A. Suto, pp. 19-57 in ref. 1.

[30] G.Scharf, W.F.Wreszinski, J.L. van Hemmen, J. Phys. A20, 4309 (1987)

[31] I.Y. Korenblit, E.F. Shender, J.E.T.P. 48, 937 (1978)

[32] M. Enz, R. Schilling, J. Phys. C19, L711 and 1765 (1986); and ref. 1, pp 59-76

[33] J. von Delft, C. Henley, Phys. Rev. Lett. 69, 3237 (1992); and Phys. Rev. B48, 965 (1993).

[34] D. Loss, D.P. DiVincenzo, G. Grinstein, Phys. Rev. Lett. 69, 3233 (1992).

[35] I. Tupitsyn, N.V. Prokof'ev, P.C.E. Stamp, Int. J. Mod. Phys. B11, 2901 (1997)

[36] E.M.Chudnovsky, L. Gunther, Phys. Rev. Lett. 60, 661 (1988)

[37] P.C.E.Stamp, E.M.Chudnovsky, B.Barbara, Int. J. Mod. Phys. B6, 1355 (1992)

[38] C. Callan. S. Coleman, Phys. Rev. D15, 2929 (1977); and Phys. Rev. D16, 1762 (1977); A.M.Polyakov, Nucl. Phys. B121, 429 (1977).

[39] A.M. Ioselevich, JETP Letts. 45, 571 (1987)

[40] A. Garg, Phys. Rev. Lett. 74, 1458 (1995)

[41] A. J. Leggett, pp 396-507 in "Matter and Chance: Proc. 1986 les Houches Summer School", ed. J. Souletie, J. Vannimenus, R. Stora, North-Holland, Amsterdam (1987); and A. J. Leggett, in "Frontiers and Borderlines in Many-Particle Physics", ed. R.A. Broglia, J.R. Schrieffer, North-Holland, Amsterdam (1988).

[42] P.C.E.Stamp, Phys. Rev. Lett. 66, 2802 (1991)

[43] G. Tatara, H. Fukuyama, J. Phys. Soc. Jap. 63, 2538 (1994); G. Tatara, H. Fukuyama, Phys. Rev. Lett. 72, $772(1994)$

[44] Bosonic oscillator models of quantum environments are very old. One can go back as far as F. Bloch, Zeit. fur Physik 81, 363 (1933), for a bosonic representation of spin waves; to S. Tomonaga, Prog. Theor. Phys. 5, 349 (1950), and D. Bohm and E.P. Gross, Phys. Rev. 75, 1851 (1949), for a representation of the low-energy excitation of Fermi systems by "collective mode" oscillators (the beginning of "bosonization"); and other similar models for phonons, photons, etc. Important early discussions of the effect of such environments on the quantum dynamics of a system coupled to them are in G.W. Ford, M. Kac, P. Mazur, J. Math. Phys. 6, 504 (1965); J. Schwinger, J. Math. Phys. 2, 407 (1961); and I.R. Senitzky, Phys. Rev. 119, 670 (1960). 
[45] For response function theory, see eg., L.D. Landau and E.M. Lifshitz, "Statistical Physics" (Pergamon); or D.Pines, P. Nozières, "Theory of Quantum Liquids", Ch. 2 and Ch. 5 (Benjamin, 1965)

[46] R.P. Feynman, A.R. Hibbs, "Quantum Mechanics and Path Integrals" (McGraw-Hill, 1965)

[47] U. Eckern, G. Schon, V. Ambegaokar, Phys. Rev. B30, 6419 (1984); G. Schon, Phys. Rev. B32, 4469 (1985).

[48] A.J. Leggett et al., Rev. Mod. Phys. 59, 1 (1987).

[49] Y. Kagan, N.V. Prokof'ev, JETP 69, 1250 (1989)

[50] "Magnetostriction: Theory and Applications of Magnetoelasticity", by E. du Tremolet de Laicheisserie (CRC Press, 1993).

[51] L.D. Landau, E.M. Lifshitz, "Electrodynamics of Continuous Media", (Pergamon).

[52] A. Abragam, B. Bleaney, "Electron Paramagnetic Resonance of Transition Ions" (Clarendon, 1970).

[53] J. Villain et al., Europhys. Lett. 27, 159 (1994)

[54] P. Politi et al., Phys. Rev. Lett. 75, 537 (1995); and Int. J. Mod. Phys. 10, 2577 (1996). See also A. Burin et al., Phys. Rev. Lett. 76, 3040 (1996), for a Comment on this paper; and the reply of Politi et al., immediately following.

[55] Y. Kagan, N.V. Prokof'ev, Ch. 2 in ref. [56].

[56] Quantum Tunneling in Condensed Matter, Elsevier Science Publishers B.V., eds. Yu. Kagan and A.J. Leggett (1992)

[57] M. Dube, P.C.E.Stamp, to be published in Int. J. Mod. Phys. B (accepted Dec. 1992); /cond-mat 9712215

[58] M. Dube, P.C.E. Stamp, preprint

[59] P.C.E. Stamp, M. Dube, E. Mueller (preprint)

[60] K. Hepp, Helv. Phys. Acta 45, 237 (1972); J.S. Bell, Helv. Phys. Acta 48, 93 (1975).

[61] H. Grabert, P. Schramm, G.-L. Ingold, Phys. Rep. 168, 115 (1988)

[62] V.B. Braginsky, V.P. Mitrofanov, V.I.Panov, "Systems with Small Dissipation", (Univ. of Chicago Press, 1985); and V.B. Braginsky, F. Ya. Khalili, "Quantum Measurement", (C.U.P., 1992).

[63] A. Schmid, Ann. Phys. 170, 336 (1986)

[64] U. Weiss, "Quantum Dissipative Systems", (World Sci., 1993)

[65] A.J. Leggett, Phys. Rev. B30, 1208 (1984)

[66] B.A. Jones, C.M.Varma, J.W. Wilkins, Phys. Rev. Lett. 61, 125 (1988), and refs. therein.

[67] P. Hanggi, P. Talkner, M. Borkovec, Rev. Mod. Phys. 62, 251 (1990) 
[68] V. Hakim, V. Ambegaokar, Phys. Rev. Phys. Rev. A32, 423 (1985)

[69] N.V. Prokof'ev, P.C.E. Stamp, /cond-mat 9511011 (unpublished).

[70] D. Gatteschi et al., Science 265, 1054 (1994)

[71] O. Kahn, "Molecular magnetism", VCH publishers (1993).

[72] I. Tupitsyn et al., (preprint)

[73] R. Sessoli et al. J. Am. Chem. Soc. 115, 1804 (1993); and R. Sessoli et al., Nature 365, 141 (1993)

[74] A. Garunin, E.M. Chudnovsky (preprint)

[75] A. Bartolome et al (preprint)

[76] N.V. Prokof'ev, P.C.E. Stamp, submitted to Phys. Rev. Letts, 2 Oct 1997 (/cond-mat 9710246).

[77] M. Novak et al. preprint

[78] A.J. Leggett, Prog. Th. Phys. Supp. 69, 80 (1980)

[79] See A. Garg, Phys. Rev Lett. 70, C2198 (1993), and reply of D. D. Awschalom et al., ibid., C2199 (1993); or A. Garg, Phys. Rev. Lett. 71, 4241 (1993), and the associated Comment of D. D. Awschalom et al., ibid., C4276 (1993); or A. Garg, Science 272, 425 (1996), with the reply of D. D. Awschalom et al., ibid., 425 (1996)

[80] D.D. Awschalom et al., Phys. Rev. Lett. 65, 783 (1990)

[81] See J. Tejada, Science 272, 424 (1996), and the reply by D. D. Awschalom et al, ibid., 425 (1996)).

[82] G. Levine, J. Howard, Phys. Rev. Let. 75, 4142 (1995)

[83] G. Levine, J. Howard, Phys. Rev. Lett. 76, 3241 (1996) 